\title{
Hadamard states for the Klein-Gordon equation on Lorentzian manifolds of bounded geometry
}

\author{
Christian GÉrard, Omar OulghazI, and Michał Wrochna
}

\begin{abstract}
We consider the Klein-Gordon equation on a class of Lorentzian manifolds with Cauchy surface of bounded geometry, which is shown to include examples such as exterior Kerr, Kerr-de Sitter spacetime and the maximal globally hyperbolic extension of the Kerr outer region. In this setup, we give an approximate diagonalization and a microlocal decomposition of the Cauchy evolution using a time-dependent version of the pseudodifferential calculus on Riemannian manifolds of bounded geometry. We apply this result to construct all pure regular Hadamard states (and associated Feynman inverses), where regular refers to the state's two-point function having Cauchy data given by pseudodifferential operators. This allows us to conclude that there is a oneparameter family of elliptic pseudodifferential operators that encodes both the choice of (pure, regular) Hadamard state and the underlying spacetime metric.
\end{abstract}

\section{INTRODUCTION \& SUMMARY OF RESULTS}

1.1. Introduction. Modern formulations of quantum field theory on curved spacetimes allow for a precise distinction between local, model-independent features, and global aspects specific to the concrete physical setup. In the case of non-interacting scalar fields, the study of the latter is directly related to the propagation of singularities for the Klein-Gordon equation, as well as to specific global properties of its solutions, such as two-point function positivity. Thus, a careful implementation of methods from microlocal analysis that takes into account asymptotic properties of the spacetime is essential in the rigorous construction of quantum fields. The present paper is aimed at generalizing known methods, in particular GW1, by providing the necessary tools to work on a much wider class of backgrounds that includes examples such as Kerr and Kerr-de Sitter spacetimes.

Before formulating the problem in more detail, let us first recall how various notions from quantum field theory are related to inverses of the Klein-Gordon operator and to special classes of bi-solutions.

\subsubsection{Klein-Gordon equation. Consider a Klein-Gordon operator}

$$
P=-\nabla^{a} \nabla_{a}+V(x)
$$

on a Lorentzian manifold $(M, g)$, where $V: M \rightarrow \mathbb{R}$ is a smooth function. Assuming global hyperbolicity $y^{1}$ of $(M, g)$, the operator $P$ has two essential properties, the proofs of which date back to Leray Le, C-B, BGP.

The first one is the existence of retarded/advanced inverses of $P$, i.e. operators $G_{\text {ret/adv }}$, mapping $C_{0}^{\infty}(M)$ into $C^{\infty}(M)$ such that

$$
P \circ G_{\mathrm{adv} / \mathrm{ret}}=G_{\mathrm{ret} / \mathrm{adv}} \circ P=\mathbb{1}, \quad \operatorname{supp} G_{\mathrm{ret} / \mathrm{adv}} u \subset J^{ \pm}(\operatorname{supp} u),
$$

2010 Mathematics Subject Classification. 81T20, 35S05, 35L05, 58J40, 53C50.

Key words and phrases. Hadamard states, pseudodifferential calculus, manifolds of bounded geometry, Feynman parametrices.

${ }^{1}$ See Subsect. 3.2 
where $J^{ \pm}(K)$ is the future/past causal shadow ${ }^{2}$ of a set $K \subset M$. The second is the unique solvability of the Cauchy problem: if $\left\{\Sigma_{t}\right\}_{t \in \mathbb{R}}$ is a foliation of $M$ by space-like Cauchy hypersurfaces, and $\rho(t): C^{\infty}(M) \ni \phi \mapsto\left(\phi \uparrow_{\Sigma_{t}}, \mathrm{i}^{-1} \partial_{n} \phi \uparrow_{\Sigma_{t}}\right) \in C^{\infty}\left(\Sigma ; \mathbb{C}^{2}\right)$ is the Cauchy data operator on $\Sigma_{t}$, then, for any fixed $s$, there exists a unique solution of the Cauchy problem

$$
\left\{\begin{array}{l}
P \phi=0, \\
\rho(s) \phi=f
\end{array}\right.
$$

for any given $f \in C_{0}^{\infty}\left(\Sigma ; \mathbb{C}^{2}\right)$ (and moreover, $\rho(t) \phi \in C_{0}^{\infty}\left(\Sigma ; \mathbb{C}^{2}\right)$ for any $t \in \mathbb{R}$ ). In the present setup the two properties are actually essentially equivalent.

These two facts are basic to the theory of quantum Klein-Gordon fields on the curved spacetime $(M, g)$, see e.g. [Di], which we now briefly recall (see Subsect. 7.1 for more details).

1.1.2. Quantum Klein-Gordon fields. By a phase space we will mean a complex vector space equipped with a non-degenerate hermitian form. The operator $G=$ $G_{\text {ret }}-G_{\text {adv }}$ is anti-hermitian for the natural scalar product $(u \mid v)_{M}=\int_{M} \bar{u} v d \operatorname{vol}_{g}$, which allows to equip $C_{0}^{\infty}(M)$ with the hermitian form $\bar{u} \cdot Q v=\mathrm{i}(u \mid G v)_{M}$. One can show that the kernel of $G$ equals to $P C_{0}^{\infty}(M)$, hence if $\mathcal{V}=\frac{C_{0}^{\infty}(M)}{P C_{0}^{\infty}(M)},(\mathcal{V}, Q)$ is a phase space - it is in fact the fundamental structure that defines the classical content of the theory.

This allows one to introduce the polynomial $\mathrm{CCR}^{*}$-algebra $\operatorname{CCR}(\mathcal{V}, Q)$, by definition generated by the identity $\mathbb{1}$ and elements called the (abstract) charged fields, which are of the form $\psi([u]), \psi^{*}([u])$ for $[u] \in \frac{C_{0}^{\infty}(M)}{P C_{0}^{\infty}(M)}$ and are subject to the relations:

$$
\begin{aligned}
\text { i) } \quad[u] \mapsto \psi([u]) \text { is } \mathbb{C} \text { - anti-linear, } \\
\text { ii) } \quad[u] \mapsto \psi^{*}([u]) \text { is } \mathbb{C} \text { - linear, } \\
\text { iii) } \quad[\psi([u]), \psi([v])]=\left[\psi^{*}([u]), \psi^{*}([v])\right]=0, \\
\quad\left[\psi([u]), \psi^{*}([v])\right]=\mathrm{i}(u \mid G v)_{M} \mathbb{1}, \\
\text { iv) } \quad \psi([u])^{*}=\psi^{*}([u]) .
\end{aligned}
$$

The algebraic approach to quantum field theory provides a way to represent the above canonical commutation relations in terms of closed operators on some concrete Hilbert space. The standard way to obtain such a representation is to specify a state.

1.1.3. Hadamard states. A state $\omega$ on $\operatorname{CCR}(\mathcal{V}, Q)$ is a positive linear functional $\omega$ on $\operatorname{CCR}(\mathcal{V}, Q)$ such that $\omega(\mathbb{1})=1$. A particularly natural class of states for linear Klein-Gordon fields is the class of quasi-free states (see e.g. [DG, Sec. 17.1] and references therein), which are entirely determined by the expectation values:

$$
\omega\left(\psi([u]) \psi^{*}([v])\right)=:\left(u \mid \Lambda^{+} v\right)_{M}, \quad \omega\left(\psi^{*}([v]) \psi([u])\right)=:\left(u \mid \Lambda^{-} v\right)_{M} .
$$

This definition implies in particular that $P \circ \Lambda^{ \pm}=\Lambda^{ \pm} \circ P=0$. It is also natural to require that $\Lambda^{ \pm}: C_{0}^{\infty}(M) \rightarrow C^{\infty}(M)$, in which case $\Lambda^{ \pm}$have distributional kernels $\Lambda^{ \pm}\left(x, x^{\prime}\right) \in \mathcal{D}^{\prime}(M \times M)$, called the two-point functions of the state $\omega$.

Among all quasi-free states, Hadamard states are considered as the physically acceptable ones, because their short distance behavior resembles that of the vacuum state on Minkowski spacetime [KW]. Since the work of Radzikowski Ra, Hadamard states are characterized by a condition on the wave front set of their twopoint functions $\Lambda^{ \pm}$, see Def. 7.3 for the precise statement. The use of wave front sets

\footnotetext{
${ }^{2}$ The future/past causal shadow of $K \subset M$ is the set of points reached from $K$ by future/past directed causal (i.e. non-spacelike) curves.
} 
had a deep impact on quantum field theory on curved spacetimes, for example on the perturbative construction of interacting models; see e.g. [BF2, Da2, [HW, KM] and also BDH, Da1, Da3] for some recent related mathematical developments.

The microlocal formulation of the Hadamard condition in $\mathrm{Ra}$ is intimately linked to the notion of distinguished parametrices introduced by Duistermaat and Hörmander in their influential paper [DH]. Distinguished parametrices are parametrices of $P$ (inverses modulo smoothing errors), which are determined uniquely (modulo smoothing errors) by the wave front set of their Schwartz kernel. Duistermaat and Hörmander demonstrated that there are exactly four classes of distinguished parametrices, the advanced/retarded and Feynman/anti-Feynman ones, see Subsect. 6.6 for details. The (uniquely defined) retarded/advanced inverses $G_{\text {ret/adv }}$ are examples of retarded/advanced parametrices.

In contrast, there is no canonical choice of a Feynman/anti-Feynman inverse on a generic spacetime. This is actually very closely related to the problem of specifying a distinguished Hadamard state, see e.g. [FV2]. More specifically, the link between Hadamard states and Feynman inverses discovered by Radzikowski is that if $\Lambda^{ \pm}$ are the two-point functions of a Hadamard state then the operator

$$
G_{\mathrm{F}}=\mathrm{i}^{-1} \Lambda^{+}+G_{\text {adv }}=\mathrm{i}^{-1} \Lambda^{-}+G_{\text {ret }}
$$

is a Feynman invers $6^{3}$ of $P$.

There already exist a large number of existence results for Hadamard states. First of all, the deformation argument of Fulling, Narcowich and Wald [FNW] shows that Hadamard states exist on any globally hyperbolic spacetime. This construction has however the disadvantage of being very indirect, which poses problems in applications. An alternative existence proof on arbitrary globally hyperbolic spacetimes was given in GW1. It has however another severe drawback which is that it fails to produce pure states (see Subsect. 7.1.1) in general.

Specific examples of Hadamard states on spacetimes with special (asymptotic) symmetries include passive states for stationary spacetimes [SV], states constructed from data at null infinity on various classes of asymptotically flat or asymptotically de Sitter spacetimes [Mo, DMP1, BJ], DW] and on cosmological spacetimes ${ }^{4}$ [DMP2, JS, BT]. Furthermore, a remarkable recent result by Sanders [Sa] proves the existence and Hadamard property of the so-called Hartle-Hawking-Israel state on spacetimes with a static bifurcate Killing horizon.

Finally, Junker [Ju1, Ju2] and Junker and Schrohe [JS] used the pseudodifferential calculus on a Cauchy hypersurface $\Sigma$ to construct Hadamard states in the case of $\Sigma$ compact. The construction was then reworked in GW1 to yield classes of Hadamard states for $P$ in the non-compact case. Let us emphasize that outside of the case of $\Sigma$ compact, the calculus of properly supported pseudodifferential operators, which exists on any smooth manifold, is not sufficient to address the positivity condition $\Lambda^{ \pm} \geq 0$ and the $C C R$ condition $\Lambda^{+}-\Lambda^{-}=\mathrm{i} G$ which have to be satisfied by the two-point functions $\Lambda^{ \pm}$in order to be consistent with 1.2 (see Subsect. 7.1.4). This was tackled in GW1 by assuming that the Cauchy surface $\Sigma$ is diffeomorphic to $\mathbb{R}^{d}$ (so that the spacetime $M$ is diffeomorphic to $\mathbb{R}^{1+d}$ ), with some uniformity conditions on $g$ at spatial infinity, which allowed to use the uniform pseudodifferential calculus on $\mathbb{R}^{d}$.

\footnotetext{
${ }^{3}$ One could call $G_{\mathrm{F}}$ a 'time-ordered Feynman inverse' to make the distinction with the Feynman propagator of Gell-Redman, Haber and Vasy GHV Va, which is a generalized inverse of $P$ considered as a Fredholm operator on suitably chosen spaces; here we just stick to the shorthand terminology.

${ }^{4}$ Let us also mention that on static and cosmological spacetimes with compact Cauchy surface, a different construction of Hadamard states was recently proposed by Brum and Fredenhagen BF1.
} 
In the case of spacetimes with Cauchy surfaces that are either compact or diffeomorphic to $\mathbb{R}^{d}$, the constructions in [Ju1, Ju2, GW1] have the advantage that they yield examples of Hadamard states which (in contrast to the general existence argument from [GW1]) are pure, and it turns out that with some additional effort it is possible to obtain large classes of those. Furthermore, the two-point functions of these states are given by rather explicit formulae (in contrast to [FNW]), from which one can recover the spacetime metric, see Subsect. 1.3 for a discussion in the present, more general context. Unfortunately, many spacetimes of interest, like for instance blackhole spacetimes fall outside the hypotheses in [Ju1, Ju2, GW1.

1.2. Content of the paper. In this paper we rework and extend the results of JJu1, Ju2, GW1 in two essential directions. First of all, we greatly generalize the framework of GW1 by basing our analysis on the pseudodifferential calculus on manifolds of bounded geometry, due to Kordyukov $\mathrm{Ko}$ and Shubin Sh2]. This allows us to work on a much larger class of spacetimes, including examples such as Kerr and Kerr-de Sitter spacetimes. Secondly, the construction of Hadamard states is now obtained as a consequence of a microlocal decomposition of the Cauchy evolution operator $\mathcal{U}_{A}(t, s)$ associated to $P$. Beside simplifying the proofs, this allows us to derive many formulas of independent interest, including for instance expressions for the Feynman inverses canonically associated to the Hadamard states we construct.

Let us now describe in more detail the content of the paper.

The background on Riemannian manifolds of bounded geometry is presented in Sect. 2. We use an equivalent definition of bounded geometry which is much more convenient in practice. In rough terms, it amounts to the existence of chart diffeomorphisms $\left\{\psi_{x}\right\}_{x \in M}$ such that the pull-back metric (on $\mathbb{R}^{n}$ ) $\left(\psi_{x}^{-1}\right)^{*} g$ is (together with all derivatives) bounded above and below and equivalent to the flat metric (together with all derivatives), uniformly w.r.t. $x \in M$.

This leads naturally to the notion of Lorentzian manifolds of bounded geometry and of Cauchy hypersurfaces of bounded geometry, developed in Sect. 3, which is an interesting topic in its own right. The main ingredient is the choice of a reference Riemannian metric $\hat{g}$ used to define bounded tensors. We then introduce in Subsect. 3.3 a class of spacetimes and associated Klein-Gordon operators for which parametrices for the Cauchy problem can be constructed by pseudodifferential calculus:

Hypothesis 1.1. We assume that there exists a neighborhood $U$ of a Cauchy surface $\Sigma$ in $(M, g)$, such that:

$(\mathrm{H})(U, g)$ is conformally embedded in a Lorentzian manifold of bounded geometry $(M, \tilde{g})$ and the conformal factor $\tilde{c}^{2}$ is such that $\nabla_{\hat{g}} \ln \tilde{c}$ is a bounded $(1,0)$ tensor, moreover $\Sigma$ is a so-called Cauchy hypersurface of bounded geometry in $(M, \tilde{g})$;

(M) $\tilde{c}^{2} V$ is a bounded (0,0)-tensor.

We refer to Subsect. 3.3 for the detailed definitions. It turns out, see Sect. 4. that most standard examples of spacetimes, like cosmological spacetimes, Kerr, Kerr-de Sitter, the maximal globally hyperbolic extension of Kerr, or cones, double cones and wedges in Minkowski space belong to this class of spacetimes.

The pseudodifferential calculus on a manifold of bounded geometry is recalled in Sect. 5. A new result of importance for the analysis in the later sections of the paper is a version of Egorov's theorem, see Thm. 5.15 .

Sect. 6 contains the main analytical results of the paper. The condition that $\Sigma$ is a Cauchy hypersurface of bounded geometry allows to identify the neighborhood $U$ with $I \times \Sigma$ (with $I$ an open interval), and the Klein-Gordon equation on $U$ can 
be reduced to the standard form

$$
\partial_{t}^{2} \phi+r(t, x) \partial_{t} \phi+a\left(t, x, \partial_{x}\right) \phi=0,
$$

where $a\left(t, x, \partial_{x}\right)$ is a second order, elliptic differential operator on $\Sigma$. Denoting by $\mathcal{U}_{A}(t, s)$ the Cauchy evolution operator for $[1.3)$, mapping $\rho(s) \phi$ to $\rho(t) \phi$, we construct what we call a microlocal decomposition of $\mathcal{U}_{A}$, i.e. a decomposition

$$
\mathcal{U}_{A}(t, s)=\mathcal{U}_{A}^{+}(t, s)+\mathcal{U}_{A}^{-}(t, s),
$$

where $\mathcal{U}_{A}^{ \pm}$have the following properties, see Thm. 6.5.

(1) $\left\{\mathcal{U}_{A}^{ \pm}(t, s)\right\}_{t, s \in I}$ are two-parameter groups (i.e. $\mathcal{U}_{A}^{ \pm}\left(t, t^{\prime}\right) \mathcal{U}_{A}^{ \pm}\left(t^{\prime}, s\right)=\mathcal{U}_{A}^{ \pm}(t, s)$ for all $\left.t, t^{\prime}, s \in I\right)$ and $\mathcal{U}_{A}^{ \pm}(t, t)=: c^{ \pm}(t)$ are projections,

(2) $\mathcal{U}_{A}^{ \pm}(t, s)$ propagate the wave front set in the upper/lower energy shells $\mathcal{N}^{ \pm}$, i.e. the two respective connected components of the characteristic set of (1.3),

(3) the kernels of $\mathcal{U}_{A}^{ \pm}(t, s)$ are symplectically orthogonal for the canonical symplectic form preserved by the evolution.

We demonstrate in Thm. 6.8 that to such a decomposition one can associate a unique Feynman inverse for $P$.

Sect. 7 is devoted to the construction of Hadamard states from a microlocal decomposition, which can be summarized as follows. We use the 'time-kernel' notation for two-point functions $\Lambda^{ \pm}$, that is we write $\Lambda^{ \pm}(t, s)$ to mean the associated operator-valued Schwartz kernel in the time variable. We say that a state is regular if $\Lambda^{ \pm}(t, t)$ is a matrix of pseudodifferential operators on $\Sigma$ for some $t$.

Theorem 1.2. Let $(M, g)$ be a spacetime satisfying Hypothesis 1.1 and consider the reduced Klein-Gordon equation (1.3). Let $t_{0} \in I$. Then there exists a pure regular Hadamard state with two-point functions given by

$$
\Lambda^{ \pm}(t, s)=\mp \pi_{0} \mathcal{U}_{A}^{ \pm}(t, s) \pi_{1}^{*},
$$

where $\pi_{0}, \pi_{1}$ are the projections to the respective two components of Cauchy data and $\left\{\mathcal{U}_{A}^{ \pm}(t, s)\right\}_{t, s \in I}$ is a microlocal decomposition, such that

$$
\mathcal{U}_{A}^{ \pm}\left(t_{0}, t_{0}\right)=\left(\begin{array}{cc}
\mp\left(b^{+}-b^{-}\right)^{-1} b^{\mp} & \pm\left(b^{+}-b^{-}\right)^{-1} \\
\mp b^{+}\left(b^{+}-b^{-}\right)^{-1} b^{-} & \pm b^{ \pm}\left(b^{+}-b^{-}\right)^{-1}
\end{array}\right)\left(t_{0}\right)
$$

for some pair $b^{ \pm}\left(t_{0}\right)$ of elliptic first order pseudodifferential operators. Moreover, the two-point functions of any pure regular Hadamard state are of this form.

The detailed results are stated in Thm. 7.8 and 7.10, see also Prop. 7.6 for the arguments that allow to get two-point functions for the original Klein-Gordon equation on the full spacetime $(M, g)$ rather than for the reduced equation $(1.3)$ on $I \times \Sigma$.

Since one can get many regular states out of a given one by applying suitable Bogoliubov transformations as in GW1, Thm. 1.2 yields in fact a large class of Hadamard states.

1.3. From quantum fields to spacetime geometry. In our approach, microlocal splittings are obtained by setting

$$
\mathcal{U}_{A}^{ \pm}(t, s):=\mathcal{U}_{A}\left(t, t_{0}\right) \mathcal{U}_{A}^{ \pm}\left(t_{0}, t_{0}\right) \mathcal{U}_{A}\left(t_{0}, s\right)
$$

where $\mathcal{U}_{A}^{ \pm}\left(t_{0}, t_{0}\right)$ is defined by formula 1.6 with $b^{ \pm}(t)$ constructed for $t \in I$ as approximate solutions (i.e. modulo smoothing terms) of the operatorial equation

$$
\left(\partial_{t}+\mathrm{i} b^{ \pm}+r\right) \circ\left(\partial_{t}-\mathrm{i} b^{ \pm}\right)=\partial_{t}^{2}+r \partial_{t}+a,
$$

and satisfying some additional conditions, see Sect. 6 (in particular Thm. 6.1) for details. We note that the approximate factorization (1.7) was already used by Junker in his construction of Hadamard states [Ju1, Ju2]. 
In summary, there is a pair of time-dependent elliptic pseudodifferential operators $b^{ \pm}(t)$ that uniquely determines the choice of a pure regular Hadamard state. It is interesting to remark that $b^{ \pm}(t)$ also determines the spacetime metric. First, by subtracting the members of (1.7) one gets $r(t)$ modulo smoothing errors. Then 1.7) gives $a(t)$ modulo smoothing terms. But since $a(t)$ and $r(t)$ are differential operators (the latter is just a multiplication operator), they can be determined exactly. Furthermore, the reduced operator on the r.h.s. of (1.7) is just the Klein-Gordon operator in Gaussian normal coordinates near a Cauchy hypersurface $\Sigma_{t_{0}}$ (see Subsect. 7.2), so the metric can be read in these coordinates from the knowledge of $a$ and $r$.

This way, both quantum fields (derived from pure Hadamard states) and the underlying spacetime metric are encoded by a time-dependent elliptic pseudodifferential operator $b^{+}(t) \oplus b^{-}(t)$. As long as one considers only pure Hadamard states (and spacetimes for which Gaussian normal coordinates make sense globally), this provides in particular a solution to the problem discussed in [ST] which consists in finding a description of Hadamard states without having to specify the spacetime metric explicitly. It would be thus interesting to try to build a theory where $b^{+}(t) \oplus b^{-}(t)$ is treated as a dynamical quantity that accounts for both quantum degrees of freedom and spacetime geometry.

We also note that the construction does not indicate directly how to select states with specific symmetries (in the case when the spacetime has any), which would be desirable for applications and therefore deserves further investigation (see e.g. [DD for some recent attempts).

1.4. Notation. - if $X, Y$ are sets and $f: X \rightarrow Y$ we write $f: X \stackrel{\sim}{\rightarrow} Y$ if $f$ is bijective. If $X, Y$ are equipped with topologies, we write $f: X \rightarrow Y$ if the map is continuous, and $f: X \stackrel{\sim}{\rightarrow} Y$ if it is a homeomorphism.

- the domain of a closed, densely defined operator $a$ will be denoted by Dom $a$.

- if $a$ is a selfadjoint operator on a Hilbert space $\mathcal{H}$, we write $a>0$ if $a \geq 0$ and Ker $a=\{0\}$. We denote by $\langle a\rangle^{s} \mathcal{H}$ the completion of Dom $|a|^{s}$ for the norm $\|u\|_{s}=\left\|\left(1+a^{2}\right)^{s / 2} u\right\|$.

- if $a, b$ are selfadjoint operators on a Hilbert space $\mathcal{H}$, we write $a \sim b$ if

$$
a, b>0, \operatorname{Dom} a^{\frac{1}{2}}=\operatorname{Dom} b^{\frac{1}{2}}, c^{-1} b \leq a \leq c b,
$$

for some constant $c>0$.

- similarly if $I \subset \mathbb{R}$ is an open interval and $\left\{\mathcal{H}_{t}\right\}_{t \in I}$ is a family of Hilbert spaces with $\mathcal{H}_{t}=\mathcal{H}$ as topological vector spaces, and $a(t), b(t)$ are two selfadjoint operators on $\mathcal{H}_{t}$, we write $a(t) \sim b(t)$ if for each $J \Subset I$ there exist constants $c_{1, J}, c_{2, J}>0$ such that

$$
a(t), b(t) \geq c_{1, J}>0, \quad c_{2, J} b(t) \leq a(t) \leq c_{2, J}^{-1} b(t), t \in J .
$$

- from now on the operator of multiplication by a function $f$ will be denoted by $f$, while the operators of partial differentiation will be denoted by $\bar{\partial}_{i}$, so that $\left[\bar{\partial}_{i}, f\right]=\partial_{i} f$.

- we set $\langle x\rangle=\left(1+x^{2}\right)^{\frac{1}{2}}$ for $x \in \mathbb{R}^{n}$.

\section{RiEmANNIAN MANIFOLDS OF BOUNDED GEOMETRY}

2.1. Definition. We recall the notion of a Riemannian manifold of bounded geometry, see [CG, Ro]. An important property of Riemannian manifolds of bounded geometry is that they admit a nice 'uniform' pseudodifferential calculus, introduced in [Sh2, $\mathrm{Ko}$, which will be recalled in Sect. 5 . 
2.1.1. Notation. We denote by $\delta$ the flat metric on $\mathbb{R}^{n}$ and by $B_{n}(y, r) \subset \mathbb{R}^{n}$ the open ball of center $y$ and radius $r$. If $(M, g)$ is a Riemannian manifold and $x \in M$ we denote by $B_{M}^{g}(x, r)$ (or $B^{g}(x, r)$ if the underlying manifold $M$ is clear from the context) the geodesic ball of center $x$ and radius $r$.

We denote by $r_{x}>0$ the injectivity radius at $x$ and by $\exp _{x}^{g}: B_{T_{x} M}^{g(x)}\left(0, r_{x}\right) \rightarrow M$ the exponential map at $x$.

If $0<r<r_{x}$ it is well known that $\exp _{x}^{g}\left(B_{T_{x} M}^{g(x)}(0, r)\right)=B_{M}^{g}(x, r)$ is an open neighborhood of $x$ in $M$. Choosing a linear isometry $e_{x}:\left(\mathbb{R}^{n}, \delta\right) \rightarrow\left(T_{x} M, g(x)\right)$ we obtain Riemannian normal coordinates at $x$ using the map $\exp _{x}^{g} \circ e_{x}$.

If $T$ is a $(q, p)$ tensor on $M$, we can define the canonical norm of $T(x), x \in M$, denoted by $\|T\|_{x}$, using appropriate tensor powers of $g(x)$ and $g^{-1}(x)$. T is bounded if $\sup _{x \in M}\|T\|_{x}<\infty$.

Let $U \Subset \mathbb{R}^{n}$ be open, relatively compact with smooth boundary. We denote by $C_{\mathrm{b}}^{\infty}(U)=C^{\infty}\left(\mathbb{R}^{n}\right) \uparrow_{U}$ the space of smooth functions on $U$, bounded with all derivatives.

If $V$ is another open set like $U$ and $\chi: U \rightarrow V$ is a diffeomorphism, we will abuse slightly notation and write that $\chi \in C_{\mathrm{b}}^{\infty}(U)$ if all components of $\chi$ belong to $C_{\mathrm{b}}^{\infty}(U)$ and all components of $\chi^{-1}$ belong to $C_{\mathrm{b}}^{\infty}(V)$.

One defines similarly smooth $(q, p)$ tensors on $U$, bounded with all derivatives. For coherence with later notation, this space will be denoted by $\mathrm{BT}_{q}^{p}(U, \delta)$, where $\delta$ is the flat metric on $U$. We equip $\mathrm{BT}_{q}^{p}(U, \delta)$ with its Fréchet space topology.

Definition 2.1. A Riemannian manifold $(M, g)$ is of bounded geometry if

(1) the injectivity radius $r_{g}:=\inf _{x \in M} r_{x}$ is strictly positive,

(2) $\nabla_{g}^{k} R_{g}$ is a bounded tensor for all $k \in \mathbb{N}$, where $R_{g}, \nabla_{g}$ are the Riemann curvature tensor and covariant derivative associated to $g$. tions.

We give an alternative characterization, which is often more useful in applica-

Theorem 2.2. A Riemannian manifold $(M, g)$ is of bounded geometry iff for each $x \in M$, there exists $U_{x}$ open neighborhood of $x$ and

$$
\psi_{x}: U_{x} \stackrel{\sim}{\longrightarrow} B_{n}(0,1)
$$

a smooth diffeomorphism with $\psi_{x}(x)=0$ such that if $g_{x}:=\left(\psi_{x}^{-1}\right)^{*} g$ then:

(C1) the family $\left\{g_{x}\right\}_{x \in M}$ is bounded in $\mathrm{BT}_{2}^{0}\left(B_{n}(0,1), \delta\right)$,

(C2) there exists $c>0$ such that:

$$
c^{-1} \delta \leq g_{x} \leq c \delta, x \in M .
$$

A family $\left\{U_{x}\right\}_{x \in M}$ resp. $\left\{\psi_{x}\right\}_{x \in M}$ as above will be called a family of good chart neighborhoods, resp. good chart diffeomorphisms.

Proof. Let us first prove the $\Rightarrow$ implication. We choose

$$
U_{x}=\exp _{x}^{g} \circ e_{x}\left(B_{n}\left(0, \frac{r}{2}\right)\right)=B_{M}^{g}\left(x, \frac{r}{2}\right),
$$

for $e_{x}: \mathbb{R}^{n} \rightarrow T_{x} M$ a linear isometry and $\psi_{x}^{-1}(v)=\exp _{x}^{g}\left(\frac{r}{2} e_{x} v\right)$ for $v \in B_{n}(0,1)$.

It is known (see e.g. [CGT, Sect. 3]) that if $(M, g)$ is of bounded geometry, then $\left\{g_{x}\right\}_{x \in M}$ is bounded in $\mathrm{BT}_{2}^{0}\left(B_{n}(0,1), \delta\right)$. In fact by [Ro, Prop. 2.4] the Christoffel symbols expressed in normal coordinates at $x$ are uniformly bounded with all derivatives. Since $\nabla_{i} g_{j k}=\partial_{i} g_{j k}-\Gamma_{i j}^{l} g_{l k}=0$, this implies that all derivatives of $g_{x}$ in normal coordinates are bounded, hence $(\mathrm{C} 1)$ holds. Moreover, by $[\mathrm{Ro}$, Lemma 2.2] we know that

$$
m_{x}:=\left(\sup _{X}\left\|\left(\exp _{x}^{g}\right)_{*} X\right\|_{g(x)}+\left\|\left(\exp _{x}^{g}\right)_{*} X\right\|_{g(x)}^{-1}\right),
$$


where $X$ ranges over all unit vector fields on $B_{n}(0,1)$, is uniformly bounded in $x \in M$. This is equivalent to property $(\mathrm{C} 2)$.

Let us now prove $\Leftarrow$. We first check that $\nabla^{k} R$ is a bounded tensor for $k \in \mathbb{N}$. Since $\psi_{x}:\left(U_{x}, g\right) \rightarrow\left(B_{n}(0,1), g_{x}\right)$ is isometric, it suffices to show that

$$
\sup _{x \in M}\left\|\nabla_{g_{x}}^{k} R_{g_{x}}(0)\right\|<\infty .
$$

In (2.1), the norm is associated to $g_{x}$, but by condition $(\mathrm{C} 2)$ we can replace it by the norm associated to the flat metric $\delta$. Then the l.h.s. of 2.1 is a fixed polynomial in the derivatives of $g_{x}$ and $g_{x}^{-1}$ computed at 0 , which are uniformly bounded in $x \in M$, by condition (C1). Therefore (2.1) holds.

It remains to prove that the injectivity radius $r_{g}$ is strictly positive. Let us denote for a moment by $r(x, N, h)$ the injectivity radius at $x \in N$ for $(N, h)$ a Riemannian manifold.

Clearly

$$
r\left(x, U_{x}, g\right) \leq r(x, M, g), \quad x \in M .
$$

By the isometry property of $\psi_{x}$ recalled above, we have:

$$
r\left(x, U_{x}, g\right)=r\left(0, B_{n}(0,1), g_{x}\right) .
$$

Conditions $(\mathrm{C} 1),(\mathrm{C} 2)$ and standard estimates on differential equations imply that $\inf _{x \in M} r\left(0, B_{n}(0,1), g_{x}\right)>0$ hence $r_{g}>0$ by 2.2 , 2.3.

Lemma 2.3. Let $\left\{U_{x}\right\}_{x \in M}$ be the good chart neighborhoods in Thm. 2.2. Then there exists $r>0$ such that $B_{M}^{g}(x, r) \subset U_{x}$ for all $x \in M$.

Proof. From condition (C2) in Thm. 2.2 we obtain the existence of some $r_{1}>0$ such that for any $x \in M B_{g_{x}}\left(0, r_{1}\right) \subset B_{n}\left(0, \frac{1}{2}\right)$. Since $\psi_{x}:\left(U_{x}, g\right) \rightarrow\left(B_{n}(0,1), g_{x}\right)$ is isometric, this implies that $B_{g}\left(x, r_{1}\right) \subset U_{x}$ as claimed.

Theorem 2.4. Let $(M, g)$ be a manifold of bounded geometry and $\epsilon<\inf \left(1, r, r_{g}\right)$, where $r$ is given in Lemma 2.3. Set

$$
\chi_{x}:=\psi_{x} \circ \exp _{x}^{g} \circ e_{x}: B_{n}(0, \epsilon) \rightarrow \psi_{x}\left(B_{M}^{g}(x, \epsilon)\right) .
$$

Then for any multi-index $\alpha$ one has:

$$
\sup _{x \in M, y \in B_{n}(0, \epsilon)}\left\|D_{y}^{\alpha} \chi_{x}(y)\right\|+\sup _{x \in M, y \in \psi_{x}\left(B_{M}^{g}(x, \epsilon)\right)}\left\|D_{y}^{\alpha} \chi_{x}^{-1}(y)\right\|<\infty .
$$

Proof. Set $V_{x}=\psi_{x}\left(B_{M}^{g}(x, \epsilon)\right)$. Since $B_{M}^{g}(x, \epsilon) \subset U_{x}$ by Lemma 2.3 , we see that $V_{x} \subset B_{n}(0,1)$. This implies 2.4 for $\alpha=0$.

Let us now consider the case $|\alpha|=1$. Since $g_{x}=\left(\psi_{x}^{-1}\right)^{*} g$, we have $\chi_{x}^{*} g_{x}=$ $\left(\exp _{x}^{g} \circ e_{x}\right)^{*} g$. Since $(M, g)$ is of bounded geometry, there exists $c>0$ such that

$$
c^{-1} \delta \leq\left(\exp _{x}^{g} \circ e_{x}\right)^{*} g \leq c \delta
$$

Using also condition (C2) of Thm. 2.2 we obtain $c_{1}>0$ such that

$$
c_{1}^{-1} \delta \leq g_{x} \leq c_{1} \delta
$$

hence

$$
c_{1}^{-1} \chi_{x}^{*} \delta \leq \chi_{x}^{*} g_{x} \leq c_{1} \chi_{x}^{*} \delta .
$$

Since $\chi_{x}^{*} g_{x}=\left(\exp _{x}^{g} \circ e_{x}\right)^{*} g$, we obtain:

$$
c_{1}^{-1}\left(\exp _{x}^{g} \circ e_{x}\right)^{*} g \leq \chi_{x}^{*} \delta \leq c_{1}\left(\exp _{x}^{g} \circ e_{x}\right)^{*} g .
$$

Combining 2.5 and (2.6) we obtain $c_{2}>0$ such that

$$
c_{2}^{-1} \delta \leq \chi_{x}^{*} \delta \leq c_{2} \delta
$$

This is equivalent to 2.4 for $|\alpha|=1$. 
To bound higher derivatives we use that $\chi_{x}$ is the exponential map transported by the chart diffeomorphism $\psi_{x}$. Denoting by $\Gamma_{i j, x}^{k}$ the Christoffel symbols for $g_{x}$, we obtain that if $v \in B_{n}(0, \epsilon) \subset T_{x} M$ and $|t| \leq 1$ and $\left(x^{1}(t), \ldots, x^{n}(t)\right):=\chi_{x}(t v)$ we have:

$$
\left\{\begin{array}{l}
\ddot{x}^{k}(t)=\Gamma_{i j, x}^{k}(x(t)) \dot{x}^{i}(t) \dot{x}^{j}(t), \\
x(0)=0 \\
\dot{x}(0)=v
\end{array}\right.
$$

Since $\left\{\Gamma_{i j, x}^{k}\right\}_{x \in M}$ is a bounded family in $C_{\mathrm{b}}^{\infty}\left(B_{n}(0,1)\right)$, it follows from standard arguments on dependence on initial conditions for differential equations that $\chi_{x}$ is uniformly bounded in $C_{\mathrm{b}}^{\infty}\left(B_{n}(0, \epsilon)\right)$ for $x \in M$. Since we already know that $D \chi_{x}^{-1}$ is bounded in $C^{0}\left(V_{x}\right)$, we also obtain that $\chi_{x}^{-1}$ is bounded in $C_{\mathrm{b}}^{\infty}\left(V_{x}\right)$ uniformly in $x \in M$ as claimed. This completes the proof of the theorem.

2.2. Chart coverings and partitions of unity. It is known (see Sh2, Lemma $1.2])$ that if $(M, g)$ is of bounded geometry, there exist coverings by good chart neighborhoods:

$$
M=\bigcup_{i \in \mathbb{N}} U_{i}, \quad U_{i}=U_{x_{i}}, x_{i} \in M
$$

which are in addition uniformly finite, i.e. there exists $N \in \mathbb{N}$ such that $\bigcap_{i \in I} U_{i}=\emptyset$ if $\sharp I>N$. Setting $\psi_{i}=\psi_{x_{i}}$, we will call $\left\{U_{i}, \psi_{i}\right\}_{i \in \mathbb{N}}$ a good chart covering of $M$.

One can associate (see [Sh2, Lemma 1.3]) to a good chart covering a partition of unity:

$$
1=\sum_{i \in \mathbb{N}} \chi_{i}^{2}, \chi_{i} \in C_{0}^{\infty}\left(U_{i}\right)
$$

such that $\left\{\left(\psi_{i}^{-1}\right)^{*} \chi_{i}\right\}_{i \in \mathbb{N}}$ is a bounded sequence in $C_{\mathrm{b}}^{\infty}\left(B_{n}(0,1)\right)$. Such a partition of unity will be called a good partition of unity.

2.3. Bounded tensors, bounded differential operators, Sobolev spaces. We now recall the definition of bounded tensors, bounded differential operators and of Sobolev spaces on $(M, g)$ of bounded geometry, see [Sh2].

\subsubsection{Bounded tensors.}

Definition 2.5. Let $(M, g)$ of bounded geometry. We denote by $\mathrm{BT}_{q}^{p}(M, g)$ the spaces of smooth $(q, p)$ tensors $T$ on $M$ such that if $T_{x}=\left(\exp _{x}^{g} \circ e_{x}\right)^{*} T$ then the family $\left\{T_{x}\right\}_{x \in M}$ is bounded in $\mathrm{BT}_{q}^{p}\left(B_{n}\left(0, \frac{r}{2}\right), \delta\right)$. We equip $\mathrm{BT}_{q}^{p}(M, g)$ with its natural Fréchet space topology.

By Thm. 2.4 we can replace in Def. 2.5 the geodesic maps $\exp _{x}^{g} \circ e_{x}$ by $\psi_{x}^{-1}$, where $\left\{\psi_{x}\right\}_{x \in M}$ is any family of good chart diffeomorphisms as in Thm. 2.2 .

The Fréchet space topology on $\mathrm{BT}_{q}^{p}(M, g)$ is independent on the choice of the family of good chart diffeomorphisms $\left\{\psi_{x}\right\}_{x \in M}$.

2.3.2. Bounded differential operators. If $m \in \mathbb{N}$ we denote by $\operatorname{Diff}^{m}\left(B_{n}(0,1), \delta\right)$ the space of differential operators of order $m$ on $B_{n}(0,1)$ with $C_{\mathrm{b}}^{\infty}\left(B_{n}(0,1)\right)$ coefficients, equipped with its Fréchet space topology.

Definition 2.6. Let $(M, g)$ of bounded geometry. We denote by $\operatorname{Diff}^{m}(M, g)$ the space of differential operators $P$ of order $m$ on $M$ such that if $P_{x}=\left(\psi_{x}^{-1}\right)^{*} P$ then the family $\left\{P_{x}\right\}_{x \in M}$ is bounded in $\operatorname{Diff}^{m}\left(B_{n}(0,1), \delta\right)$. 
2.3.3. Sobolev spaces. Let $-\Delta_{g}$ be the Laplace-Beltrami operator on $(M, g)$, defined as the closure of its restriction to $C_{0}^{\infty}(M)$.

Definition 2.7. For $s \in \mathbb{R}$ we define the Sobolev space $H^{s}(M, g)$ as:

$$
H^{s}(M, g):=\left\langle-\Delta_{g}\right\rangle^{-s / 2} L^{2}(M, d g),
$$

with its natural Hilbert space topology.

It is known (see e.g. [Ko, Sect. 3.3]) that if $\left\{U_{i}, \psi_{i}\right\}_{i \in \mathbb{N}}$ is a good chart covering and $1=\sum_{i} \chi_{i}^{2}$ is a subordinate good partition of unity, then an equivalent norm on $H^{s}(M, g)$ is given by:

$$
\|u\|_{s}^{2}=\sum_{i \in \mathbb{N}}\left\|\left(\psi_{i}^{-1}\right)^{*} \chi_{i} u\right\|_{H^{s}\left(B_{n}(0,1)\right)}^{2} .
$$

2.4. Embeddings of bounded geometry. We now recall the definition of embeddings of bounded geometry, see [El].

Definition 2.8. Let $(M, g)$ an $n$-dimensional Riemannian manifold of bounded geometry, $\Sigma$ an $n-1$ dimensional manifold. An embedding $i: \Sigma \rightarrow M$ is called of bounded geometry if there exists a family $\left\{U_{x}, \psi_{x}\right\}_{x \in M}$ of good chart diffeomorphisms for $g$ such that if $\Sigma_{x}:=\psi_{x}\left(i(\Sigma) \cap U_{x}\right)$ for we have

$$
\Sigma_{x}=\left\{\left(v^{\prime}, v_{n}\right) \in B_{n}(0,1): v_{n}=F_{x}\left(v^{\prime}\right)\right\},
$$

where $\left\{F_{x}\right\}_{x \in M}$ is a bounded family in $C_{\mathrm{b}}^{\infty}\left(B_{n-1}(0,1)\right)$.

The following fact is shown in [El, Lemma 2.27].

Lemma 2.9. Assume $i: \Sigma \rightarrow M$ is an embedding of bounded geometry of $\Sigma$ in $(M, g)$. Then $\left(\Sigma, i^{*} g\right)$ is of bounded geometry.

Lemma 2.10. Let $i: \Sigma \rightarrow M$ an embedding of bounded geometry. Then there exists a family $\left\{U_{x}, \psi_{x}\right\}_{x \in M}$ of good chart diffeomorphisms as in Def. 2.8 such that if $x \in i(\Sigma)$ one has

$$
\Sigma_{x}=\psi_{x}\left(i(\Sigma) \cap U_{x}\right)=\left\{\left(v^{\prime}, v_{n}\right) \in B_{n}(0,1): v_{n}=0\right\} .
$$

Proof. Since the family $\left\{F_{x}\right\}_{x \in \Sigma}$ in Def. 2.8 is uniformly bounded in $C_{0}^{\infty}\left(B_{n-1}(0,1)\right)$ we can find $\alpha, \beta>0$ such that if $\phi_{x}\left(v^{\prime}, v_{n}\right)=\left(v^{\prime}, \alpha\left(v_{n}-F_{x}\left(v^{\prime}\right)\right)\right)$ we have $B_{n}(0,1) \subset$ $\phi_{x}\left(B_{n}(0,1)\right) \subset B_{n}(0, \beta)$. Clearly $\left\{\phi_{x}\right\}_{x \in \Sigma}$ is a bounded family of diffeomorphisms in $C_{\mathrm{b}}^{\infty}\left(B_{n}(0,1)\right)$. For $x \in \Sigma$ we replace $U_{x}$ by $\left(\phi_{x} \circ \psi_{x}\right)^{-1} B_{n}(0,1)$ and $\psi_{x}$ by $\phi_{x} \circ \psi_{x}$. For $x \notin \Sigma, U_{x}$ and $\psi_{x}$ are left unchanged.

2.5. Equivalence classes of Riemannian metrics. The results of this subsection are due to $\mathrm{Ou}$.

Proposition 2.11. Let $(M, g)$ be of bounded geometry. Let $k$ be another Riemannian metric on $M$ such that $k \in \mathrm{BT}_{2}^{0}(M, g)$ and $k^{-1} \in \mathrm{BT}_{0}^{2}(M, g)$. Then

(1) $(M, k)$ is of bounded geometry;

(2) $\operatorname{BT}_{q}^{p}(M, g)=\mathrm{BT}_{q}^{p}(M, k), H^{s}(M, g)=H^{s}(M, k)$ as topological vector spaces. Let us write $k \sim g$ if the above conditions are satisfied. Then $\sim$ is an equivalence relation on the class of bounded geometry Riemannian metrics on $M$.

Proof. Let us first prove (1). We equip $M$ with a good chart covering $\left\{U_{x}, \psi_{x}\right\}_{x \in M}$ for $g$. Then conditions (C1), (C2) of Thm. 2.2 are satisfied by $k$, hence $(M, k)$ is of bounded geometry and $\left\{U_{x}, \psi_{x}\right\}_{x \in M}$ is a good chart covering for $k$. Using that $k \in \mathrm{BT}_{2}^{0}(M, g)$ and $k^{-1} \in \mathrm{BT}_{0}^{2}(M, g)$ this implies that $\mathrm{BT}_{q}^{p}(M, g)=\mathrm{BT}_{q}^{p}(M, k)$. The statement about Sobolev spaces follows from the equivalent norm given in (2.7). 
Let us show that $\sim$ is symmetric. If $g_{1} \sim g_{2}$, then we have seen that $\mathrm{BT}_{q}^{p}\left(M, g_{1}\right)=$ $\mathrm{BT}_{q}^{p}\left(M, g_{2}\right)$. Since $\left(M, g_{2}\right)$ is of bounded geometry, we have $g_{2} \in \mathrm{BT}_{2}^{0}\left(M, g_{2}\right)=$ $\operatorname{BT}_{2}^{0}\left(M, g_{1}\right), g_{2}^{-1} \in \operatorname{BT}_{0}^{2}\left(M, g_{2}\right)=\operatorname{BT}_{0}^{2}\left(M, g_{1}\right)$, hence $g_{2} \sim g_{1}$. The same argument shows that $\sim$ is transitive.

We conclude this subsection with an easy fact.

Proposition 2.12. Let $g_{i}, i=1,2$ be two Riemannian metrics of bounded geometry having a common family of good chart diffeomorphisms $\left\{U_{x}, \psi_{x}\right\}_{x \in M}$. Then $g_{1} \sim$ $g_{2}$.

Proof. This follows directly from the remark below Def. 2.5 and the definition of the equivalence relation $g_{1} \sim g_{2}$. $\square$

2.6. Examples. We now recall some well-known examples of manifolds of bounded geometry, which will be useful later on.

2.6.1. Compact manifolds and compact perturbations. Clearly any compact Riemannian manifold is of bounded geometry. Similarly if $\left(M, g_{1}\right)$ is of bounded geometry and if $g_{2}=g_{1}$ outside some compact set, then $\left(M, g_{2}\right)$ is of bounded geometry and $g_{1} \sim g_{2}$.

2.6.2. Gluing of Riemannian manifolds. Let $\left(M_{i}, g_{i}\right), i=1,2$ be two Riemannian manifolds of bounded geometry, $K_{i} \subset M_{i}$ be open and relatively compact and $j: K_{1} \rightarrow K_{2}$ an isometry. Then the Riemannian manifold $(M, g)$ obtained by gluing $M_{1}$ and $M_{2}$ along $K_{1} \stackrel{j}{\sim} K_{2}$ is of bounded geometry.

2.6.3. Cartesian products. If $\left(M_{i}, g_{i}\right) i=1,2$ are Riemannian manifolds of bounded geometry then $\left(M_{1} \times M_{2}, g_{1} \oplus g_{2}\right)$ is of bounded geometry.

2.6.4. Warped products. We provide a further useful argument that gives manifolds of bounded geometry in the form of warped products.

Proposition 2.13. Let $(K, h)$ be a Riemannian manifold of bounded geometry, and $M=\mathbb{R}_{s} \times K, g=d s^{2}+F^{2}(s) h$, where:

(1) $F(s) \geq c_{0}>0, \forall s \in \mathbb{R}$, for some $c_{0}>0$;

(2) $\left|F^{(k)}(s)\right| \leq c_{k} F(s), \forall s \in \mathbb{R}, k \geq 1$.

Then $(M, g)$ is of bounded geometry.

Proof. Let $r$ the injectivity radius of $(K, h)$, and $e_{y}:\left(\mathbb{R}^{n-1}, \delta\right) \rightarrow\left(T_{y} K, h(y)\right)$ for $y \in K$ be linear isometries. We set for $x=(\sigma, y) \in M$ and $c_{0}$ the constant in (1):

We have:

$$
\begin{aligned}
\left.\psi_{x}^{-1}: \quad\right]-1,1\left[\times B_{n-1}\left(0, \frac{r}{2} c_{0}\right) \rightarrow\right]-1,1\left[\times B_{K}^{h}\left(y, \frac{r c_{0}}{2 F(\sigma)}\right)\right. \\
(s, v) \mapsto\left(s+\sigma, \exp _{y}^{h}\left(F(\sigma)^{-1} e_{y} v\right)\right) .
\end{aligned}
$$

$$
g_{x}=\left(\psi_{x}^{-1}\right)^{*} g=d s^{2}+\frac{F^{2}(s+\sigma)}{F^{2}(\sigma)} h_{y}\left(e_{y} F(\sigma)^{-1} v\right) d v^{2},
$$

where $h_{y}=\left(\exp _{y}^{h} \circ e_{y}\right)^{*} h$. By (2) we have $\left|\ln \left(\frac{F(s+\sigma)}{F(\sigma)}\right)\right| \leq \int_{\sigma}^{s+\sigma}\left|\frac{F^{\prime}(u)}{F(u)}\right| d u \leq c_{1}|s|$, hence:

$$
\left.\mathrm{e}^{-c_{1}} \leq \frac{F(s+\sigma)}{F(\sigma)} \leq \mathrm{e}^{c_{1}}, \sigma \in \mathbb{R}, s \in\right]-1,1[.
$$

This implies that $g_{x}$ is uniformly equivalent to the flat metric $\delta$. Moreover from conditions (1), (2) we obtain that $\left\{g_{x}\right\}_{x \in M}$ is bounded in $\mathrm{BT}_{2}^{0}(]-1,1\left[\times B_{n-1}\left(0, \frac{r}{2} c_{0}\right)\right)$.

We now choose $\rho>0$ such that $\left.B_{n}(0, \rho) \subset\right]-1,1\left[\times B_{n-1}\left(0, \frac{r}{2} c_{0}\right)\right.$, and compose $\psi_{x}^{-1}$ with a fixed diffeomorphism between $B_{n}(0, \rho)$ and $B_{n}(0,1)$. Conditions $(\mathrm{C} 1)$, (C2) of Thm. 2.2 are then satisfied. 


\section{LORENTZIAN MANIFOLDS OF BOUNDED GEOMETRY}

In this section we consider Lorentzian manifolds $(M, g)$. Reference Riemannian metrics on $M$ with be denoted by $\hat{g}$. We still denote by $\exp _{x}^{g}$ the exponential map at $x \in M$ for $g$. The results of this section are due to $\mathrm{Ou}]$.

\subsection{Definitions.}

Definition 3.1. A smooth Lorentzian manifold $(M, g)$ is of bounded geometry if there exists a Riemannian metric $\hat{g}$ on $M$ such that:

(1) $(M, \hat{g})$ is of bounded geometry;

(2) $g \in \mathrm{BT}_{2}^{0}(M, \hat{g})$ and $g^{-1} \in \mathrm{BT}_{0}^{2}(M, \hat{g})$.

Clearly the above conditions only depend on the equivalence class of $\hat{g}$ for the equivalence relation $\sim$ introduced in Subsect. 2.5. The following theorem is a partial converse to this property.

Theorem 3.2. Let $(M, g)$ a Lorentzian manifold and $\hat{g}_{i}, i=1,2$ two Riemannian metrics on $M$ such that:

(i) $\left(M, \hat{g}_{i}\right)$ is of bounded geometry;

(ii) $g \in \mathrm{BT}_{2}^{0}\left(M, \hat{g}_{i}\right)$ and $g^{-1} \in \mathrm{BT}_{0}^{2}\left(M, \hat{g}_{i}\right)$.

Then the following are equivalent:

(1) $\hat{g}_{1} \sim \hat{g}_{2}$,

(2) there exists $c>0$ such that $c^{-1} \hat{g}_{2}(x) \leq \hat{g}_{1}(x) \leq c \hat{g}_{2}(x), \forall x \in M$,

(3) there exists $c>0$ such that $\hat{g}_{2}(x) \leq c \hat{g}_{1}(x), \forall x \in M$.

Proof. We start by some preparations. Let $(M, g)$ be a smooth Lorentzian manifold and $\hat{g}$ a Riemannian metric on $M$ such that $(M, \hat{g})$ is of bounded geometry and $g \in \mathrm{BT}_{2}^{0}(M, \hat{g}), g^{-1} \in \mathrm{BT}_{0}^{2}(M, \hat{g})$. Let $\left\{U_{x}, \psi_{x}\right\}_{x \in M}$ be a family of good chart diffeomorphisms for $\hat{g}$ and let $g_{x}=\left(\psi_{x}^{-1}\right)^{*} g$.

By the above property of $g$ and $g^{-1}$, we obtain that there exists $0<r, r^{\prime}<1$ such that $\exp _{0}^{g_{x}}$ is well defined on $B_{n}(0, r)$, is a smooth diffeomorphism on its image, and moreover $B_{n}\left(0, r^{\prime}\right) \subset \exp _{0}^{g_{x}} B_{n}(0, r)$, and the family $\left\{\exp _{0}^{g_{x}}\right\}_{x \in M}$ is bounded in $C_{\mathrm{b}}^{\infty}\left(B_{n}(0, r)\right)$.

Let us identify $B^{\hat{g}(x)}(0,1) \subset T_{x} M$ with $B_{n}(0,1) \subset \mathbb{R}^{n}$ with isometries $e_{x}$ : $\left(T_{x} M, \hat{g}(x)\right) \rightarrow\left(\mathbb{R}^{n}, \delta\right)$ and set

$$
\phi_{x}: B_{n}(0,1) \ni v \mapsto \exp _{x}^{g} \circ e_{x}(r v) \in M,
$$

$V_{x}:=\phi_{x}\left(B_{n}(0,1)\right), \chi_{x}=\phi_{x}^{-1}$. Since $\exp _{0}^{g_{x}}$ equals $\exp _{x}^{g}$ transported by $\psi_{x}$, it follows from the properties of $\left\{\exp _{0}^{g_{x}}\right\}_{x \in M}$ shown above that $\left\{V_{x}, \chi_{x}\right\}_{x \in M}$ is a family of good chart diffeomorphisms for $\hat{g}$.

Let now $\hat{g}_{i}, i=1,2$ as in the theorem and let $r=\inf \left(r_{1}, r_{2}\right)$, where $r_{i}$ is the radius $r$ above for $\hat{g}_{i}$. We choose isometries $e_{i, x}:\left(\mathbb{R}^{n}, \delta\right) \rightarrow\left(T_{x} M, \hat{g}_{i}(x)\right)$ and denote by $\left\{V_{i, x}, \chi_{i, x}\right\}_{x \in M}$ the families of good chart diffeomorphisms for $\hat{g}_{i}$ constructed above.

Let us compute the map $T_{x}:=\chi_{1, x} \circ \chi_{2, x}^{-1}$, which is defined on some neighborhood of 0 in $B_{n}(0,1)$. Denoting by $\lambda_{r}: \mathbb{R}^{n} \rightarrow \mathbb{R}^{n}$ the multiplication by $r$, we have:

$$
\begin{aligned}
\chi_{1, x} \circ \chi_{2, x}^{-1} & =\lambda_{r} \circ\left(\exp _{x}^{g} \circ e_{1, x}\right)^{-1} \circ\left(\lambda_{r} \circ\left(\exp _{x}^{g} \circ e_{2, x}\right)^{-1}\right)^{-1} \\
& =\lambda_{r} \circ e_{1, x}^{-1} \circ e_{2, x} \circ \lambda_{r}^{-1}=e_{1, x}^{-1} \circ e_{2, x} .
\end{aligned}
$$

We claim that property (1) is equivalent to

$$
\sup _{x \in M}\left\|T_{x}\right\|+\left\|T_{x}^{-1}\right\|<\infty
$$

where $\|\cdot\|$ is the norm on $L\left(\mathbb{R}^{n}\right)$ inherited from $\delta$. To prove the claim we set:

$$
\hat{g}_{i, x}=\left(\chi_{1, x}^{-1}\right)^{*} \hat{g}_{i}, \quad \tilde{g}_{i, x}=\left(\chi_{i, x}^{-1}\right)^{*} \hat{g}_{i},
$$


so that

$$
\tilde{g}_{2, x}=T_{x}^{*} \hat{g}_{2, x}
$$

We have seen above that $\left\{\chi_{i, x}\right\}_{x \in M}$ is a family of good chart diffeomorphisms for $\hat{g}_{i}$. Therefore $\left\{\tilde{g}_{2, x}\right\}_{x \in M}$ and $\left\{\tilde{g}_{2, x}^{-1}\right\}_{x \in M}$ are bounded in $\mathrm{BT}_{2}^{0}\left(B_{n}(0,1), \delta\right)$ and $\mathrm{BT}_{0}^{2}\left(B_{n}(0,1), \delta\right)$. Moreover by Prop. 2.11 we know that $\hat{g}_{2} \sim \hat{g}_{1}$ iff $\left\{\hat{g}_{2, x}\right\}_{x \in M}$ and $\left\{\hat{g}_{2, x}^{-1}\right\}_{x \in M}$ are bounded in $\mathrm{BT}_{2}^{0}\left(B_{n}(0,1), \delta\right)$ and $\mathrm{BT}_{0}^{2}\left(B_{n}(0,1), \delta\right)$. By (3.3), this is equivalent to the fact that $\left\{\left(T_{x}^{-1}\right)^{*} \delta\right\}_{x \in M}$ and $\left\{\left(T_{x}^{-1}\right)^{*} \delta^{-1}\right\}$ are bounded in $\mathrm{BT}_{2}^{0}\left(B_{n}(0,1), \delta\right)$ and $\mathrm{BT}_{0}^{2}\left(B_{n}(0,1), \delta\right)$. Since $T_{x}$ are linear maps this is clearly equivalent to 3.2 , which completes the proof of the claim.

Let now $g_{i, x}:=\left(\chi_{i, x}^{-1}\right)^{*} g=\left(\lambda_{r}^{-1}\right)^{*}\left(e_{i, x}\right)^{*}\left(\exp _{x}^{g}\right)^{*} g$. The same argument as in (3.1) shows that:

$$
g_{2, x}=T_{x}^{*} g_{1, x}={ }^{\mathrm{t}} T_{x} \circ g_{1, x} \circ T_{x} .
$$

Computing the determinant of the quadratic forms $g_{i, x}(0)$ using $\delta$, this implies that

$$
\left(\operatorname{det} T_{x}\right)^{2}=\operatorname{det} g_{2, x}(0) \operatorname{det} g_{1, x}^{-1}(0) .
$$

Since $g_{i}$ and $g_{i}^{-1}$ are bounded tensors, we obtain that there exists $c>0$ such that $c^{-1} \leq\left|\operatorname{det} T_{x}\right| \leq c$. This implies that 3.2 is equivalent to

$$
\sup _{x \in M}\left\|T_{x}^{-1}\right\|<\infty .
$$

Finally the discussion above shows that property (2) is equivalent to $c^{-1} \tilde{g}_{2, x} \leq$ $\hat{g}_{2, x} \leq c \tilde{g}_{2, x} \forall x \in M$, which is equivalent to (3.2). Property (3) is equivalent to $\hat{g}_{2, x} \leq c \tilde{g}_{2, x} \forall x \in M$ which is equivalent to 3.4 . Since we have seen that (1), 3.2 and (3.4) are equivalent, the proof is complete.

3.2. Cauchy hypersurfaces of bounded geometry. We adopt the convention that a spacetime $(M, g)$ is a Hausdorff, paracompact, connected time orientable Lorentzian manifold equipped with a time orientation. Lorentzian manifolds are naturally endowed with a causal structure; we refer the reader to [Wa, Chap. 8] or [BGP, Sect. 1.3] for details.

In the sequel we denote by $I_{M}^{ \pm, g}(U),\left(\operatorname{resp} . J_{M}^{ \pm, g}(U)\right)$ for $U \subset M$ the future/past time-like (resp. causal) shadow of $U$. If $(M, g)$ is clear from the context we use instead the notation $I^{ \pm}(U)$ (resp. $J^{ \pm}(U)$ ). We denote by $C_{\mathrm{sc}}^{\infty}(M)$ the space of smooth space-compact functions, i.e. with support included in $J^{+}(K) \cup J^{-}(K)$ for some compact set $K \Subset M$.

A smooth hypersurface $\Sigma$ is a Cauchy hypersurface if any inextensible piecewise smooth time-like curve intersects $\Sigma$ at one and only one point.

A spacetime having a Cauchy hypersurface is called globally hyperbolic (see BS] for the equivalence with the alternative definition where $\Sigma$ is not required to be smooth). Global hyperbolic spacetimes are natural Lorentzian manifolds on which to study Klein-Gordon operators.

Definition 3.3. Let $(M, g)$ be an $n$-dimensional Lorentzian manifold of bounded geometry and $\hat{g}$ a Riemannian metric as in Def. 3.1. Assume also that $(M, g)$ is globally hyperbolic. Let $\Sigma \subset M$ a spacelike Cauchy hypersurface. Then $\Sigma$ is called $a$ bounded geometry Cauchy hypersurface if:

(1) the injection $i: \Sigma \rightarrow M$ is of bounded geometry for $\hat{g}$,

(2) if $n(y)$ for $y \in \Sigma$ is the future directed unit normal for $g$ to $\Sigma$ one has:

$$
\sup _{y \in \Sigma} n(y) \cdot \hat{g}(y) n(y)<\infty .
$$

We recall now a well-known result about geodesic normal coordinates to a Cauchy hypersurface $\Sigma$. 
Proposition 3.4. Let $\Sigma$ be a space-like Cauchy hypersurface in a globally hyperbolic spacetime $(M, g)$. Then there exists a neighborhood $U$ of $\{0\} \times \Sigma$ in $\mathbb{R} \times \Sigma$ and a neighborhood $V$ of $\Sigma$ in $M$ such that the map:

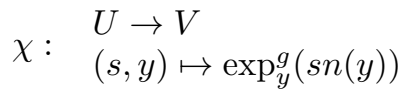

is a diffeomorphism. Moreover $\chi^{*} g\left\lceil_{V}=-d s^{2}+h_{s}\right.$ where $h_{s}$ is a smooth, $s$-dependent family of Riemannian metrics on $\Sigma$.

Proof. The first statement is shown in ['N1, Prop. 26]. To prove the second statement, we can work near a point in $\Sigma$ and introduce local coordinates $y$ on $\Sigma$. In [Wa, Sect. 3.3] it is shown that the normal geodesics are orthogonal to the hypersurfaces $\Sigma_{t}=\{s=t\}$. Since in the normal coordinates, $\partial_{s}$ is a tangent vector to the normal geodesics, and $\partial_{y^{i}}$ span $T \Sigma_{t}$ this implies that the metric does not contain $d s d y^{i}$ terms. If $n$ is the future directed normal vector field to the family $\Sigma_{t}$, then $n \cdot g n=-1$ first on $\Sigma_{0}$ and then on all $\Sigma_{t}$ by the geodesic equation. This completes the proof.

In the next theorem we study properties of the normal coordinates for Cauchy hypersurfaces of bounded geometry.

Theorem 3.5. Let $(M, g)$ a Lorentzian manifold of bounded geometry and $\Sigma$ a bounded geometry Cauchy hypersurface. Then the following holds:

(1) there exists $\delta>0$ such that the normal geodesic flow to $\Sigma$ :

$$
\begin{aligned}
\chi: \quad]-\delta, \delta[\times \Sigma \rightarrow M \\
\quad(s, y) \mapsto \exp _{y}^{g}(\operatorname{sn}(y))
\end{aligned}
$$

is well defined and is a smooth diffeomorphism on its image;

(2) $\chi^{*} g=-d s^{2}+h_{s}$, where $\left\{h_{s}\right\}_{s \in]-\delta, \delta[}$ is a smooth family of Riemannian metrics on $\Sigma$ with

$$
\begin{aligned}
\text { i) } & \left(\Sigma, h_{0}\right) \text { is of bounded geometry, } \\
\text { ii) } & s \mapsto h_{s} \in C_{\mathrm{b}}^{\infty}(]-\delta, \delta\left[, \mathrm{BT}_{2}^{0}\left(\Sigma, h_{0}\right)\right), \\
\text { iii) } & s \mapsto h_{s}^{-1} \in C_{\mathrm{b}}^{\infty}(]-\delta, \delta\left[, \mathrm{BT}_{0}^{2}\left(\Sigma, h_{0}\right)\right) .
\end{aligned}
$$

Proof. Let us first prove (1). The proof consists of several steps.

Step 1: since $g$ is of bounded geometry for the reference metric $\hat{g}$, we first see by standard arguments that there exists $\rho_{2}, c_{2}>0$ such that for all $x \in M$,

$$
\exp _{x}^{g}: B_{T_{x} M}^{\hat{g}(x)}\left(0, \rho_{2}\right) \rightarrow M
$$

is well defined and $c_{2}$-Lipschitz if we equip $B_{T_{x} M}^{\hat{g}(x)}\left(0, \rho_{2}\right)$ with the distance associated to $\hat{g}(x)$ and $M$ with the distance associated with $\hat{g}$.

Step 2: Recall that $i: \Sigma \rightarrow M$ is the natural injection. For $y \in \Sigma$, we set $A_{y}=D_{(0, y)} \chi \in L\left(\mathbb{R} \times T_{y} \Sigma, T_{y} M\right)$. We have:

$$
\begin{aligned}
& A_{y}(\alpha, v)=\alpha n(y)+D_{y} i v, \alpha \in \mathbb{R}, v \in T_{y} \Sigma \\
& A_{y}^{-1} w=\left(-n(y) \cdot g(y) w,\left(D_{y} i\right)^{-1}(w+n(y) \cdot g(y) w n(y))\right) .
\end{aligned}
$$

If we equip $T_{y} \Sigma$ with the metric $i^{*} \hat{g}(y)$ and $T_{y} M$ with $\hat{g}(y)$, we deduce from conditions (1) and (2) in Def. 3.3 that the norms of $A_{y}$ and $A_{y}^{-1}$ are uniformly bounded in $y$.

By the local inversion theorem, there exists $\delta_{1}>0$ such that for any $y \in \Sigma \chi$ is well defined on $]-\delta_{1}, \delta_{1}\left[\times B^{\hat{g}}\left(y, \delta_{1}\right) \cap \Sigma\right.$ and is a diffeomorphism on its image.

Step 3: let now $c_{1}=\sup _{y \in \Sigma} n(y) \cdot \hat{g}(y) n(y)<\infty$ and

$$
\delta=\min \left(\delta_{1}, \rho_{2}\right)\left(2+2 c_{1}+4 c_{1} c_{2}\right)^{-1},
$$


where $\rho_{2}, c_{2}$ are introduced at the beginning of the proof. We claim that

$$
\chi:]-\delta, \delta[\times \Sigma \rightarrow M
$$

is a smooth diffeomorphism on its image, which will complete the proof of (1). By the above discussion, $\chi$ is a local diffeomorphism, so it remains to prove that $\chi$ is injective. Let $\left.\left(s_{i}, y_{i}\right) \in\right]-\delta, \delta\left[\times \Sigma\right.$ such that $\chi\left(s_{1}, y_{1}\right)=\chi\left(s_{2}, y_{2}\right)=x$.

If $y \in \Sigma$ and $|s|<\delta$, we have $\|s n(y)\|_{\hat{g}} \leq c_{1} \delta<\rho_{2}$, hence by the Lipschitz property of $\exp _{x}^{g}$ in Step 1 we have:

$$
d_{\hat{g}}\left(y, \exp _{y}^{g}(\operatorname{sn}(y))\right) \leq c_{2}|s|\|n(y)\|_{\hat{g}}
$$

This yields

$$
\begin{aligned}
d_{\hat{g}}\left(y_{1}, y_{2}\right) & \leq d_{\hat{g}}\left(y_{1}, x\right)+d_{\hat{g}}\left(y_{2}, x\right) \\
& =d_{\hat{g}}\left(y_{1}, \exp _{y_{1}}^{g}\left(s_{1} n_{y_{1}}\right)\right)+d_{\hat{g}}\left(y_{2}, \exp _{y_{2}}^{g}\left(s_{2} n_{y_{2}}\right)\right) \\
& \leq c_{2}\left|s_{1}\right|\left\|n_{y_{1}}\right\|_{\hat{g}}+c_{2}\left|s_{2}\right|\left\|n_{y_{2}}\right\|_{\hat{g}} \\
& \leq 2 c_{2} c_{1} \delta \leq \frac{\delta_{1}}{2}
\end{aligned}
$$

It follows that $\left.\left(s_{i}, y_{i}\right) \in\right]-\delta_{1}, \delta_{1}\left[\times B^{\hat{g}}\left(y_{1}, \delta_{1}\right) \cap \Sigma\right.$. Since by Step $2 \chi$ is injective on this set, we have $\left(s_{1}, y_{1}\right)=\left(s_{2}, y_{2}\right)$, which completes the proof of $(1)$.

Let us now prove $(2)$. For $x \in \Sigma$, we choose $U_{x}, \psi_{x}$ as in Lemma 2.10. We recall that $\Sigma_{x}=\psi_{x}\left(\Sigma \cap U_{x}\right), g_{x}=\left(\psi_{x}^{-1}\right)^{*} g$ and denote by $n_{x}$ the future-directed unit normal vector field to $\Sigma_{x}$ for $g_{x}$. We have $\Sigma_{x}=\left\{v \in B_{n}(0,1): v_{n}=0\right\} \sim$ $B_{n-1}(0,1)$ and we can decompose $n_{x}$ as $n_{x}=n_{x}^{\prime}+\lambda_{x} e_{n}$, where $n_{x}^{\prime}$ is tangent to $\Sigma_{x}$. Then $\left\{g_{x}\right\}_{x \in \Sigma},\left\{g_{x}^{-1}\right\}_{x \in \Sigma},\left\{n_{x}^{\prime}\right\}_{x \in \Sigma}, \lambda_{x}$ are bounded in $\mathrm{BT}_{2}^{0}\left(B_{n}(0,1), \delta\right)$, $\mathrm{BT}_{0}^{2}\left(B_{n}(0,1), \delta\right), \mathrm{BT}_{0}^{1}\left(B_{n-1}(0,1), \delta\right)$ and $\mathrm{BT}_{0}^{0}\left(B_{n-1}(0,1), \delta\right)$ respectively.

By standard estimates on differential equations, this implies that there exists $\delta^{\prime}>0$ such that the normal geodesic flow

$$
\begin{gathered}
\left.\chi_{x}: \quad\right]-\delta^{\prime}, \delta^{\prime}\left[\times B_{n-1}\left(0, \frac{1}{2}\right) \rightarrow B_{n}(0,1)\right. \\
\left(s, v^{\prime}\right) \mapsto \exp _{\left(v^{\prime}, 0\right)}^{g_{x}}\left(s n_{x}\left(v^{\prime}, 0\right)\right)
\end{gathered}
$$

is a diffeomorphism on its image, with $\left\{\chi_{x}\right\}_{x \in \Sigma}$ bounded in $C_{\mathrm{b}}^{\infty}(]-\delta^{\prime}, \delta^{\prime}\left[\times B_{n-1}\left(0, \frac{1}{2}\right)\right)$. Moreover if $V_{x}:=\chi_{x}(]-\delta^{\prime}, \delta^{\prime}\left[\times B_{n-1}\left(0, \frac{1}{2}\right)\right)$, then $\chi_{x}^{-1}$ is the restriction to $V_{x}$ of a map $\phi_{x}: B_{n}(0,1) \rightarrow \mathbb{R}^{n}$ such that $\left\{\phi_{x}\right\}_{x \in \Sigma}$ is bounded in $C_{\mathrm{b}}^{\infty}\left(B_{n}(0,1)\right)$.

We have $\chi_{x}^{*} g_{x}=-d s^{2}+h_{x}\left(s, v^{\prime}\right) d v^{\prime 2}$, where $h_{x}\left(s, v^{\prime}\right) d v^{\prime 2}$ is an $s$-dependent Riemannian metric on $B_{n-1}(0,1)$.

To prove statement (2) it remains to check that $\left\{h_{x}\right\}_{x \in \Sigma}$ and $\left\{h_{x}^{-1}\right\}_{x \in \Sigma}$ are bounded in $\mathrm{BT}_{2}^{0}(]-\delta^{\prime}, \delta^{\prime}\left[\times B_{n-1}\left(0, \frac{1}{2}\right)\right)$ and $\mathrm{BT}_{0}^{2}(]-\delta^{\prime}, \delta^{\prime}\left[\times B_{n-1}\left(0, \frac{1}{2}\right)\right)$ respectively. This follows from the same properties of $g_{x}, g_{x}^{-1}$ and $\chi_{x}$ recalled above. The proof is complete.

Remark 3.6. Since the diffeomorphisms $\chi_{x}$ in 3.5 are bounded with all derivatives (in good coordinates for the reference Riemannian metric $\hat{g}$ ), we see that $\chi^{*} \hat{g}$ is equivalent to $d s^{2}+h_{0} d y^{2}$ on $I \times \Sigma$, or more precisely that one can extend $\chi^{*} \hat{g}$ to $\mathbb{R} \times \Sigma$ such that the extension is equivalent to $d s^{2}+h_{0} d y^{2}$ on $\mathbb{R} \times \Sigma$.

3.3. A framework for Klein-Gordon operators. In Sects. 6, 7we will consider Klein-Gordon operators on a globally hyperbolic spacetime $(M, g)$ :

$$
P=-\nabla^{a} \nabla_{a}+V, V \in C^{\infty}(M ; \mathbb{R}),
$$

and in particular the Cauchy problem on a Cauchy hypersurface $\Sigma$. In this subsection we formulate a rather general framework which will allow us later on to apply 
tools from the pseudodifferential calculus on manifolds of bounded geometry, see Sect. 5 for the construction of parametrices for the Cauchy problem for $P$.

If $\left(M_{i}, g_{i}\right)$ are two spacetimes, a spacetime embedding $i:\left(M_{1}, g_{1}\right) \rightarrow\left(M_{2}, g_{2}\right)$ is by definition an embedding that is isometric and preserves the time-orientation. In addition, if $\left(M_{i}, g_{i}\right)$ globally hyperbolic, one says that $i$ is causally compatible if:

$$
I_{M_{1}}^{ \pm, g_{1}}(U)=i^{-1}\left(I_{M_{2}}^{ \pm, g_{2}}(U)\right), \forall U \subset M_{1}
$$

We fix a globally hyperbolic spacetime $(M, g)$, a Cauchy hypersurface $\Sigma$ and a function $V \in C^{\infty}(M ; \mathbb{R})$. We assume that there exist:

(1) a neighborhood $U$ of $\Sigma$ in $M$,

(2) a Lorentzian metric $\tilde{g}$ on $M$,

(3) a function $\tilde{c} \in C^{\infty}(M ; \mathbb{R}), \tilde{c}>0$,

such that:

(H1) $\left(M, \tilde{c}^{2} \tilde{g}\right)$ is globally hyperbolic, $i:(U, g) \rightarrow\left(M, \tilde{c}^{2} \tilde{g}\right)$ is causally compatible,

(H2) $\tilde{g}$ is of bounded geometry for some reference Riemannian metric $\hat{g}, \Sigma$ is a

Cauchy hypersurface of bounded geometry in $(M, \tilde{g})$,

(H3) $d \ln \tilde{c}$ belongs to $\operatorname{BT}_{1}^{0}(M, \hat{g})$,

(M) $\tilde{c}^{2} V$ belongs to $\mathrm{BT}_{0}^{0}(M, \hat{g})$.

Proposition 3.7. Assume hypotheses $(\mathrm{H})$. Then there exist:

(1) an open interval $I$ with $0 \in I$, a diffeomorphism $\chi: I \times \Sigma \rightarrow U$,

(2) a smooth family $\left\{h_{t}\right\}_{t \in I}$ of Riemannian metrics on $\Sigma$ with

$\left(\Sigma, h_{0}\right)$ is of bounded geometry,

$$
I \ni t \mapsto h_{t} \in C_{\mathrm{b}}^{\infty}\left(I ; \mathrm{BT}_{2}^{0}\left(\Sigma, h_{0}\right)\right), I \ni t \mapsto h_{t}^{-1} \in C_{\mathrm{b}}^{\infty}\left(I ; \mathrm{BT}_{0}^{2}\left(\Sigma, h_{0}\right)\right),
$$

(3) a function $c \in C^{\infty}(I \times \Sigma), c>0$ with

$$
\nabla_{h_{0}} \ln c \in C_{\mathrm{b}}^{\infty}\left(I ; \mathrm{BT}_{0}^{1}\left(\Sigma, h_{0}\right)\right), \partial_{t} \ln c \in C_{\mathrm{b}}^{\infty}\left(I ; \mathrm{BT}_{0}^{0}\left(\Sigma, h_{0}\right)\right),
$$

or equivalently

$$
d c \in \mathrm{BT}_{1}^{0}\left(I \times \Sigma, d t^{2}+h_{0}\right),
$$

such that

$$
\chi^{*} g=c^{2}(t, y)\left(-d t^{2}+h_{t}(y) d y^{2}\right) \text { on } U \text {. }
$$

If moreover hypothesis (M) holds then:

$$
c^{2} V \circ \chi^{-1} \in C_{\mathrm{b}}^{\infty}\left(I ; \mathrm{BT}_{0}^{0}\left(\Sigma, h_{0}\right)\right) .
$$

Proof. We apply Thm. 3.5 to $\tilde{g}$ to obtain $I, U, \chi$. We set $c=\tilde{c} \circ \chi$, so that (3.7) follows from $g=\tilde{c}^{2} \tilde{g}$ on $U$. Property (2) of $t \mapsto h_{t}$ follow from Thm. 3.5, property (3) of $c$ from hypothesis (H3) and the fact that $\chi^{*} \hat{g}$ is equivalent to $d t^{2}+h_{0} d y^{2}$ on $I \times \Sigma$, by Remark 3.6. Finally 3.8 follows from hypothesis $(\mathrm{M})$.

The following proposition is a converse to Prop. 3.7 .

Proposition 3.8. Let $(M, \tilde{g})$ be a globally hyperbolic spacetime with

$$
M=\mathbb{R}_{t} \times \Sigma_{y}, \tilde{g}=-d t^{2}+h_{t}(y) d y^{2},
$$

such that $\Sigma$ is a Cauchy hypersurface in $(M, \tilde{g})$. Let $c \in C^{\infty}(M), c>0$ and $W \in C^{\infty}(M ; \mathbb{R})$. Assume that conditions (2), (3) and identity (3.7) in Prop. 3.7 are satisfied by $\left\{h_{t}\right\}_{t \in I}, c$ for some bounded open interval $I$ and $\chi=\mathrm{Id}$.

Then for any $J \Subset I$ conditions (H1), (H2), (H3) are satisfied for $g=c^{2} \tilde{g}, \tilde{c}=c$ and $U=J \times \Sigma$. If moreover $\tilde{V} \in C^{\infty}(M ; \mathbb{R})$ is such that 3.8 is satisfied for $\chi=\mathrm{Id}$, then there exist $V \in C^{\infty}(M ; \mathbb{R})$ such that $V=\tilde{V}$ on $J \times \Sigma$ and $V$ satisfies condition $(M)$. 
Proof. We extend the maps $t \mapsto h_{t}$ and $t \mapsto c(t, \cdot)$ from $I$ to $\mathbb{R}$, in such a way that conditions (2) and (3)are satisfied with $I$ replaced by $\mathbb{R}$, taking $h_{t}=h_{0}$, $c(t, \cdot)=c(0, \cdot)$ for $|t|$ large. As reference Riemannian metric on $M$ we take $\hat{g}=$ $d t^{2}+h_{t}(y) d y^{2}$. The fact that $(M, \hat{g})$ is of bounded geometry is easy. The remaining conditions in (H2), (H3) follow immediately from (2) and (3). If (3.8) holds, we can similarly construct $V$ with $V=\tilde{V}$ on $I \times \Sigma, V=0$ for $|t|$ large such that $V$ satisfies (M).

\section{Examples}

In this section we give several examples of spacetimes to which the framework of Subsect. 3.3 applies.

4.1. Cosmological spacetimes. Let $(\Sigma, h)$ a Riemannian manifold, a $\in C^{\infty}(\mathbb{R} ; \mathbb{R})$ and consider $M=\mathbb{R}_{t} \times \Sigma_{y}$ with metric

$$
g=-d t^{2}+\mathrm{a}^{2}(t) h_{i j}(y) d y^{i} d y^{j} .
$$

If $(\Sigma, h)$ is of bounded geometry, $(M, g)$ satisfies conditions $(\mathrm{H})$ for $\Sigma=\{t=0\}$, $\tilde{c}=1, U=I \times \Sigma, I \Subset \mathbb{R}$ an interval. Condition $(\mathrm{M})$ is satisfied in particular for $V=m^{2}, m \in \mathbb{R}$.

Remark 4.1. The construction of propagators and Hadamard states for KleinGordon equations on cosmological spacetimes can be done without the pseudodifferential calculus used in Sects. 6, 7 in the general case. Instead one can rely on the functional calculus for $\epsilon=\left(-\Delta_{h}\right)^{\frac{1}{2}}$. All objects constructed in Sects. 6. 7, like the propagators $\mathcal{U}_{A}^{ \pm}(t, s)$ (see Subsect. 6.5 ) or the covariances $\lambda^{ \pm}(t)$ (see Thm. 7.8) can be written as functions of $(t, s)$ and of the selfadjoint operator $\epsilon$. This amounts to what is known in the physics literature as the mode decomposition, see e.g. [JS, Ol, BT, $\mathrm{Av}$, for related results.

\subsection{Kerr and Kerr-de Sitter exterior spacetimes.}

4.2.1. The Kerr-de Sitter family. Let us recall the family of Kerr-de Sitter metrics. One sets $M=\mathbb{R}_{t} \times I_{r} \times \mathbb{S}_{\theta, \varphi}^{2}$, where $I$ is some open interval and $\theta \in[0, \pi], \varphi \in \mathbb{R} / 2 \pi \mathbb{Z}$ are the spherical coordinates on $\mathbb{S}^{2}$. The metric is given in the coordinates $(t, r, \theta, \varphi)$ (Boyer-Lindquist coordinates) by:

$$
\begin{aligned}
g= & \rho^{2}\left(\frac{d r^{2}}{\Delta_{r}}+\frac{d \theta^{2}}{\Delta_{\theta}}\right)+\frac{\Delta_{\theta} \sin ^{2} \theta}{(1+\alpha)^{2} \rho^{2}}\left(\mathrm{adt} t^{2}-\left(r^{2}+\mathrm{a}^{2}\right) d \varphi\right)^{2} \\
& -\frac{\Delta_{r}}{(1+\alpha)^{2} \rho^{2}}\left(d t-\operatorname{asin}^{2} \theta d \varphi\right)^{2} \\
= & : g_{t t} d t^{2}+g_{\varphi \varphi} d \varphi^{2}+2 g_{t \varphi} d t d \varphi+g_{r r} d r^{2}+g_{\theta \theta} d \theta^{2},
\end{aligned}
$$

for

$$
\begin{aligned}
& \Delta_{r}=\left(1-\frac{\alpha}{a^{2}} r^{2}\right)\left(r^{2}+a^{2}\right)-2 M r \\
& \Delta_{\theta}=1+\alpha \cos ^{2} \theta, \rho^{2}=r^{2}+a^{2} \cos ^{2} \theta \\
& \sigma^{2}=\left(r^{2}+a^{2}\right)^{2} \Delta_{\theta}-a^{2} \Delta_{r} \sin ^{2} \theta .
\end{aligned}
$$

Here $\alpha=\frac{\Lambda \mathrm{a}^{2}}{3}, M, \mathrm{a}, \Lambda>0$ are respectively the mass of the blackhole, its angular momentum and the cosmological constant. The Kerr metric corresponds to $\Lambda=0$.

If $\Lambda=0$ (Kerr) one assumes that $|a|<M$ (slow Kerr) which implies that for $r_{h}=M+\sqrt{M^{2}-a^{2}}$ one has:

$$
\left.r_{h}>0, \Delta_{r}\left(r_{h}\right)=0, \Delta_{r}>0 \text { on }\right] r_{h},+\infty[
$$


and one takes $I=] r_{h},+\infty[$. If $\Lambda \neq 0$ (Kerr-de Sitter) one assumes that there exists $r_{h}<r_{c}$ such that

$$
\begin{aligned}
\text { i) } & \left.r_{h}>0, \Delta_{r}>0 \text { on }\right] r_{h}, r_{c}\left[, \Delta_{r}\left(r_{h}\right)=\Delta_{r}\left(r_{c}\right)=0,\right. \\
\text { ii) } & \partial_{r} \Delta_{r}\left(r_{h}\right)>0, \partial_{r} \Delta_{r}\left(r_{c}\right)<0, \\
\text { iii) } & \sup _{\left[r_{h}, r_{c}[\right.} \Delta_{r}>\sup _{[0, \pi]} \Delta_{\theta},
\end{aligned}
$$

and one takes $I=] r_{h}, r_{c}[$. The set $S$ of parameters $(a, M, \Lambda)$ such that such $r_{h}, r_{c}$ exist is open and contains the set $\{|a|<M, \Lambda=0\}$ (slow Kerr) and $\left\{\mathrm{a}=0,9 \Lambda M^{2}<1\right\}$ (Schwarzschild-de Sitter).

It is easy to check that if $(a, M, \Lambda) \in S$ then there exists $c>0$ such that $\sigma^{2}(r, \theta) \geq c$ for all $\theta \in[0, \pi]$.

The part of the boundary $r=r_{h}$ of $M$ is the (outer) black hole horizon, the part $r=r_{c}$ in the Kerr-de Sitter case is the cosmological horizon. Condition iii) means that the region $\Delta_{r}>\Delta_{\theta}$ where $\frac{\partial}{\partial t}$ is time-like is not empty; one chooses the time orientation so that $\frac{\partial}{\partial t}$ is future oriented in this region. The spacetime $M$ is usually called the outer region of the Kerr or Kerr-de Sitter spacetime.

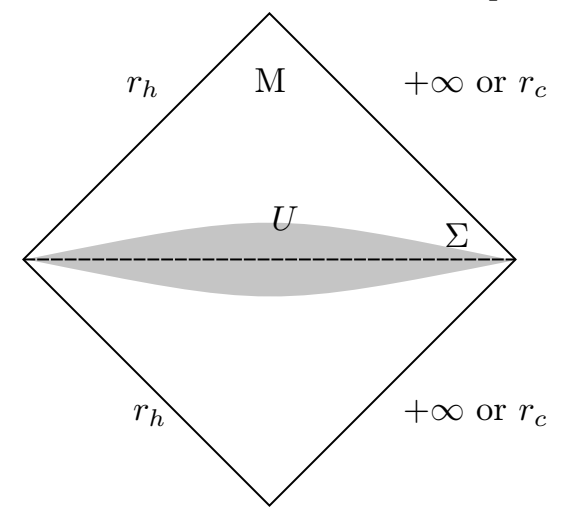

Fig. 1 Kerr-de Sitter exterior region

4.2.2. Verification of conditions (H). The first step consists in expressing the metric in rotating coordinates. We have:

$$
g=\left(g_{t t}-g_{t \varphi}^{2} g_{\varphi \varphi}^{-1}\right) d t^{2}+g_{\varphi \varphi}\left(d \varphi+g_{t \varphi} g_{\varphi \varphi}^{-1} d t\right)^{2}+g_{r r} d r^{2}+g_{\theta \theta} d \theta^{2} .
$$

We set $R=g_{t \varphi} g_{\varphi \varphi}^{-1}, \tilde{\varphi}=\varphi+t R(r, \theta)$. Denoting again $\tilde{\varphi}$ by $\varphi$ we obtain:

$$
g=\left(g_{t t}-g_{t \varphi}^{2} g_{\varphi \varphi}^{-1}\right) d t^{2}+g_{\varphi \varphi}\left(d \varphi-t \partial_{r} R d r-t \partial_{\theta} R d \theta\right)^{2}+g_{r r} d r^{2}+g_{\theta \theta} d \theta^{2} .
$$

Then one introduces Regge-Wheeler coordinates on $I$, defining $s=s(r)$ by

$$
\frac{d s}{d r}=(1+\alpha) \frac{r^{2}+\mathrm{a}^{2}}{\Delta_{r}} .
$$

(The integration constant is irrelevant). The spacetime $M$ becomes $\mathbb{R}_{t} \times \mathbb{R}_{s} \times \mathbb{S}_{\omega}^{2}$ and we choose the Cauchy hypersurface:

$$
\Sigma=M \cap\{t=0\} \sim \mathbb{R}_{s} \times \mathbb{S}_{\omega}^{2} .
$$

We set now:

$$
\tilde{c}^{2}:=-g_{t t}+g_{t \varphi}^{2} g_{\varphi \varphi}^{-1}
$$

and write

$$
g=\tilde{c}^{2} \tilde{g} \text { for } \tilde{g}=-d t^{2}+h_{t}, h_{t} \text { Riemannian metric on } \Sigma,
$$

with $h_{t}=: h_{0}-2 t \hat{h}_{1}+t^{2} \hat{h}_{2}$.

Proposition 4.2. (1) $\left(\Sigma, h_{0}\right)$ is of bounded geometry; 
(2) for $J=[-\epsilon, \epsilon]$ and $\epsilon>0$ small enough one has

$$
J \ni t \mapsto h_{t} \in C_{\mathrm{b}}^{\infty}\left(J ; \mathrm{BT}_{2}^{0}\left(\Sigma, h_{0}\right)\right), J \ni t \mapsto h_{t}^{-1} \in C_{\mathrm{b}}^{\infty}\left(J ; \mathrm{BT}_{0}^{2}\left(\Sigma, h_{0}\right)\right),
$$

(3) One has

$$
\nabla_{h_{0}} \ln \tilde{c} \in \mathrm{BT}_{0}^{1}\left(\Sigma, h_{0}\right), \tilde{c} \in \mathrm{BT}_{0}^{0}\left(\Sigma, h_{0}\right) .
$$

Remark 4.3. By Prop. 3.8 we see that conditions (H) are satisfied. Moreover $V=m^{2}$ satisfies condition (M).

Some technical computations used in the proof of Prop. 4.2 are collected in Subsect. A.1. where the reader can also find the definitions of the function classes $S_{\mathrm{KdS}}^{p}$ and $S_{\mathrm{K}}^{m}, p$, see Def. A.4

Proof of Prop. 4.2, A routine computation gives:

$$
\tilde{c}^{2}=\frac{\Delta_{r} \Delta_{\theta} \rho^{2}}{(1+\alpha)^{2} \sigma^{2}}, g_{\varphi \varphi}=\frac{\sin ^{2} \theta \sigma^{2}}{(1+\alpha)^{2} \rho^{2}}, g_{r r}=\frac{\rho^{2}}{\Delta_{r}}, g_{\theta \theta}=\frac{\rho^{2}}{\Delta_{\theta}} .
$$

We set also:

$$
F(s):=(1+\alpha)^{2} \frac{\left(r^{2}+\mathrm{a}^{2}\right)^{2}}{\Delta_{r}}, \quad G(s, \theta):=\frac{\sigma^{2}}{\left(r^{2}+\mathrm{a}^{2}\right)^{2} \Delta_{\theta}},
$$

and

$$
d \omega^{2}=d \theta^{2}+\frac{1+\alpha \cos ^{2} \theta}{1+\alpha} \sin ^{2} \theta d \varphi^{2}
$$

By Lemma A.2 $d \omega^{2}$ is a smooth Riemannian metric on $\mathbb{S}^{2}$. From the identity in Lemma A.2 we have

$$
\begin{aligned}
h_{0} & =\frac{\sigma^{2}}{\left(r^{2}+a^{2}\right)^{2} \Delta_{\theta}} d s^{2}+\frac{(1+\alpha)^{2} \sigma^{2}}{\Delta_{r} \Delta_{\theta} \rho^{2}}\left(g_{\theta \theta} d \theta^{2}+g_{\varphi \varphi} d \varphi^{2}\right) \\
& =G(s, \theta)\left(d s^{2}+\frac{F(s)}{\Delta_{\theta}} d \omega^{2}+F(s) w\right),
\end{aligned}
$$

for

$$
w=\left(\frac{a^{2}}{(1+\alpha) \rho^{2}}+\frac{2 m a^{2} r}{(1+\alpha)^{2} \rho^{4}}\right)\left(\sin ^{2} \theta d \varphi\right)^{2} \in \mathrm{T}_{2}^{0}(\Sigma) .
$$

From Lemma A.7 $v$ ) we obtain that $\inf F(s)>0$ and $\left|\partial_{s}^{\alpha} F(s)\right| \leq C_{\alpha} F(s)$, hence if $k_{0}=d s^{2}+F(s) d \omega^{2},\left(\Sigma, k_{0}\right)$ is of bounded geometry by Prop. 2.13

Next we see from Lemma A.7 vi) that $G, G^{-1} \in \mathrm{BT}_{0}^{0}\left(\Sigma, k_{0}\right)$ since $\inf G>0$ and $\partial_{s}^{\alpha}\left(F(s)^{-\frac{1}{2}} \partial_{\omega}\right)^{\beta} G$ is bounded on $\Sigma$ for any $(\alpha, \beta) \in \mathbb{N}^{3}$.

The factor in front of $\left(\sin ^{2} \theta d \varphi\right)^{2}$ in $w$ belongs to $S_{\mathrm{KdS}}^{0}$ resp. to $S_{\mathrm{K}}^{-2,0}$. The same argument as the one used for $G$, using the estimates in Lemma A.7 shows that $F(s) w \in \mathrm{BT}_{2}^{0}\left(\Sigma, k_{0}\right)$. This implies that $h_{0} \in \mathrm{BT}_{2}^{0}\left(\Sigma, k_{0}\right)$. Since $w \geq 0$ we immediately have that $h_{0}^{-1} \in \mathrm{BT}_{0}^{2}\left(\Sigma, k_{0}\right)$, i.e. $h_{0} \sim k_{0}$, which proves $(1)$.

To prove (2) we need to compute $\hat{h}_{1}$ and $\hat{h}_{2}$. We have:

$$
\begin{aligned}
\hat{h}_{1} & =\tilde{c}^{-2} g_{\varphi \varphi} R_{r} d r d \varphi+\tilde{c}^{-2} g_{\varphi \varphi} R_{\theta}(\sin 2 \theta d \theta) d \varphi \\
& =\frac{\sigma^{4}}{\Delta_{r} \Delta_{\theta} \rho^{4}} R_{r} d r\left(\sin ^{2} \theta d \varphi\right)+\frac{\sigma^{4}}{\Delta_{r} \Delta_{\theta} \rho^{4}} R_{\theta}(\sin 2 \theta d \theta)\left(\sin ^{2} \theta d \varphi\right) \\
& =\frac{\sigma^{4}}{\Delta_{\theta} \rho^{4}(1+\alpha)\left(r^{2}+a^{2}\right)} R_{r} d s\left(\sin ^{2} \theta d \varphi\right)+\frac{\sigma^{4}}{\Delta_{r} \Delta_{\theta} \rho^{4}} R_{\theta}(\sin 2 \theta d \theta)\left(\sin ^{2} \theta d \varphi\right) \\
& =: h_{1, s \varphi} d s\left(\sin ^{2} \theta d \varphi\right)+h_{1, \theta \varphi}(\sin 2 \theta d \theta)\left(\sin ^{2} \theta d \varphi\right) .
\end{aligned}
$$


Similarly:

$$
\begin{aligned}
\hat{h}_{2}= & \tilde{c}^{-2} g_{\varphi \varphi}\left(R_{r}\right)^{2} d r^{2}+\tilde{c}^{-2} g_{\varphi \varphi}\left(R_{\theta}\right)^{2}(\sin 2 \theta d \theta)^{2}+2 \tilde{c}^{-2} g_{\varphi \varphi} R_{r} R_{\theta} d r(\sin 2 \theta d \theta) \\
= & \frac{\sigma^{4}}{\Delta_{r} \Delta_{\theta} \rho^{4}} \sin ^{2} \theta\left(R_{r}\right)^{2} d r^{2}+\frac{\sigma^{4}}{\Delta_{r} \Delta_{\theta} \rho^{4}} \sin ^{2} \theta\left(R_{\theta}\right)^{2}(\sin 2 \theta d \theta)^{2} \\
& +2 \frac{\sigma^{4}}{\Delta_{r} \Delta_{\theta} \rho^{4}} \sin ^{2} \theta R_{r} R_{\theta} d r(\sin 2 \theta d \theta) \\
= & \frac{\sigma^{4}}{\Delta_{\theta} \rho^{4}\left(r^{2}+a^{2}\right)^{2}(1+\alpha)^{2}} \sin ^{2} \theta \Delta_{r}\left(R_{r}\right)^{2} d s^{2}+\frac{\sigma^{4}}{\Delta_{r} \Delta_{\theta} \rho^{4}} \sin ^{2} \theta\left(R_{\theta}\right)^{2}(\sin 2 \theta d \theta)^{2} \\
& +2 \frac{\sigma^{4}}{(1+\alpha)\left(r^{2}+a^{2}\right) \Delta_{\theta} \rho^{4}} \sin ^{2} \theta R_{r} R_{\theta} d s(\sin 2 \theta d \theta) \\
= & : h_{2, s s} d s^{2}+h_{2, \theta \theta}(\sin 2 \theta d \theta)^{2}+2 h_{2, s \theta} d s(\sin 2 \theta d \theta) .
\end{aligned}
$$

We now collect the properties of the coefficients of $\hat{h}_{1}, \hat{h}_{2}$. From A.1 and estimates similar to those in Lemma A.7 we obtain:

$$
\begin{aligned}
& h_{1, s \varphi} \in S_{\mathrm{KdS}}^{0}, \text { resp. } \in S_{\mathrm{K}}^{-1,0}, h_{1, \theta \varphi} \in S_{\mathrm{KdS}}^{0}, \text { resp. } \in S_{\mathrm{K}}^{0,0}, \\
& h_{2, s s} \in S_{\mathrm{KdS}}^{-1}, \text { resp. } \in S_{\mathrm{K}}^{-4,-1}, h_{2, \theta \theta} \in S_{\mathrm{KdS}}^{-1}, \text { resp. } \in S_{\mathrm{K}}^{-2,-1}, \\
& h_{2, s \theta} \in S_{\mathrm{KdS}}^{-1}, \text { resp. } \in S_{\mathrm{K}}^{-3,-1} .
\end{aligned}
$$

Since $\sin 2 \theta d \theta$ and $\sin ^{2} \theta d \varphi$ are smooth forms on $\mathbb{S}^{2}$, this implies that $\hat{h}_{i} \in \mathrm{BT}_{2}^{0}\left(\Sigma, h_{0}\right)$, $i=1,2$. If $J=[-\epsilon, \epsilon]$ for $\epsilon$ small enough we have hence

$$
J \ni t \mapsto h_{t} \in C_{\mathrm{b}}^{\infty}\left(J, \mathrm{BT}_{2}^{0}\left(\Sigma, h_{0}\right)\right), J \ni t \mapsto h_{t}^{-1} \in C_{\mathrm{b}}^{\infty}\left(J, \mathrm{BT}_{0}^{2}\left(\Sigma, h_{0}\right)\right),
$$

which proves (2).

From 4.2 we obtain that $\tilde{c}^{2} \in S_{\mathrm{KdS}}^{-1}$, resp. $\in S_{\mathrm{K}}^{0,-1}$. This implies (3).

4.3. Kerr-Kruskal spacetime. In this subsection we consider the maximal globally hyperbolic extension of the outer Kerr region considered in Subsect. 4.2. For the sake of brevity we call it the Kerr-Kruskal extension. In the slow Kerr case $(|\mathrm{a}|<M, \Lambda=0), \Delta_{r}$ has two roots $0<r_{-}<r_{+},\left(r_{+}\right.$was previously denoted by $\left.r_{h}\right)$. The region $r>r_{+}$of $\mathbb{R}_{t} \times \mathbb{R}_{r} \times \mathbb{S}_{\omega}^{2}$ considered earlier is called the (Boyer-Lindquist) block I, the region $r_{-}<r<r_{+}$is called the block II.

The construction of the Kerr-Kruskal extension of block I is as follows (see O’N2, Chap. 2] for details): a block II is glued to the future of block I along $r=r_{+}, t>0$ using Kerr-star coordinates, and a block II', i.e. a block II with reversed time orientation, is glued to the past of block I along $r=r_{+}, t<0$ using star-Kerr coordinates. Then a block I', i.e. a block I with reversed time orientation, is glued to the past of block II and the future of block II'. The four blocks can be smoothly glued together at $r=t=0$ (the so-called crossing sphere), see ['N2, Sect. 3.4]. The time orientation of block I can be extended to a global time orientation, and it can be shown that the resulting spacetime $\left(M^{\text {ext }}, g\right)$ is globally hyperbolic, with $\Sigma^{\text {ext }}=\{t=0\}$ as a Cauchy hypersurface. 


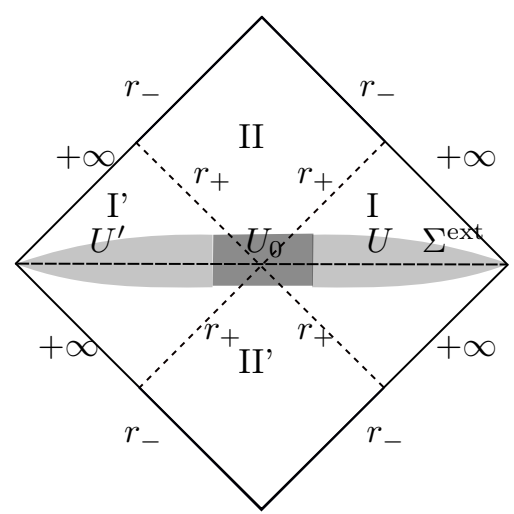

Fig. 2 Kerr-Kruskal extension

We claim that the Kerr-Kruskal extension $M^{\text {ext }}$ satisfies the conditions (H). In fact let $U$ be a neighborhood of $\Sigma$ in block I of the form $\{|t|\langle\epsilon, r>R\}$, such that Prop. 4.2 holds on $[-\epsilon, \epsilon]$, and let $U^{\prime}$ be its copy in block I'. We also fix a relatively compact neighborhood $U_{0}$ of the crossing sphere such that $V=U^{\prime} \cup U_{0} \cup U$ is a neighborhood of $\Sigma^{\text {ext }}$ in $M^{\text {ext }}$. It is clear that the hypotheses of Prop. 3.8 are satisfied, since they are satisfied over $U$ and $U^{\prime}$, and $U_{0}$ is relatively compact.

4.4. Double cones, wedges and lightcones in Minkowski. In this subsection we consider the Klein-Gordon operator $P=-\nabla^{a} \nabla_{a}+m^{2}$ on double cones, wedges and lightcones in Minkowski spacetime.

\subsubsection{Double cones.}

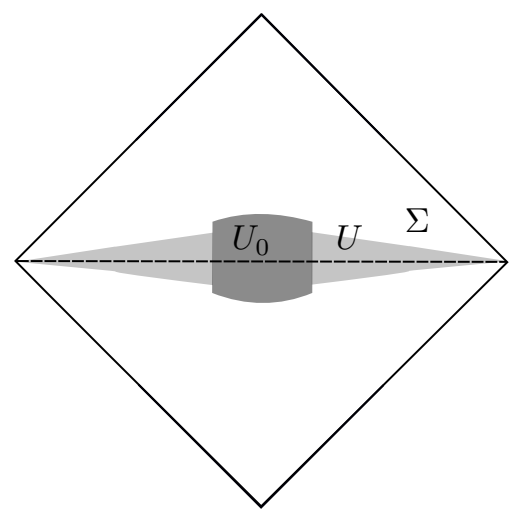

Fig. 3 The double cone

The standard double cone is

$$
M=\left\{(t, x) \in \mathbb{R}^{1+d}:|t|<1-|x|\right\}, d s^{2}=-d t^{2}+d x^{2} .
$$

We follow the framework of Subsect. 3.3 with $\Sigma=M \cap\{t=0\}, V=m^{2}$. We set

$$
U=\left\{|t|<\delta(1-|x|), t^{2}+(1-|x|)^{2}<\delta\right\} \text { for } 0<\delta \ll 1,
$$

and fix a relatively compact open set $U_{0}$ such that $U \cup U_{0}$ is a neighborhood of $\Sigma$, see Fig. 3. It suffices to check conditions $(\mathrm{H})$ over $U$, since $U_{0}$ is relatively compact in $M$. We introduce polar coordinates $x=r \omega$ and set

$$
r=1-\mathrm{e}^{-X} \cos T, \quad t=\mathrm{e}^{-X} \sin T .
$$

We are reduced to

$$
\begin{aligned}
U & =]-\alpha, \alpha\left[T_{T} \times\right] C,+\infty\left[X \times \mathbb{S}_{\omega}^{d-1}, \Sigma=\{T=0\},\right. \\
d s^{2} & =\mathrm{e}^{-2 X} \cos (2 T)\left(-d T^{2}+d X^{2}+2 \tan (2 T) d T d X+\left(\mathrm{e}^{X}-\cos T\right)^{2} d \omega^{2}\right)
\end{aligned}
$$


We take $\tilde{c}(T, X)=\mathrm{e}^{-X} \cos (2 T)$ and choose the reference Riemannian metric

$$
\hat{g}=d T^{2}+d X^{2}+\mathrm{e}^{2 X} d \omega^{2},
$$

which is of bounded geometry by Prop. 2.13. The Lorentzian metric

$$
\tilde{g}=-d T^{2}+d X^{2}+2 \tan (2 T) d T d X+\left(\mathrm{e}^{X}-\cos T\right)^{2} d \omega^{2}
$$

is of bounded geometry for $\hat{g}$. Clearly $\Sigma=\{T=0\}$ is a bounded hypersurface of $(U, \hat{g})$. Its normal vector field for $\tilde{g}$ is $\partial_{T}$, from which it follows that $\Sigma$ is a Cauchy surface of bounded geometry, hence (H2) is satisfied. One easily checks that $\tilde{c}$ satisfies (H3) and that (M) is satisfied for $V=m^{2}$.

\subsubsection{Wedges.}

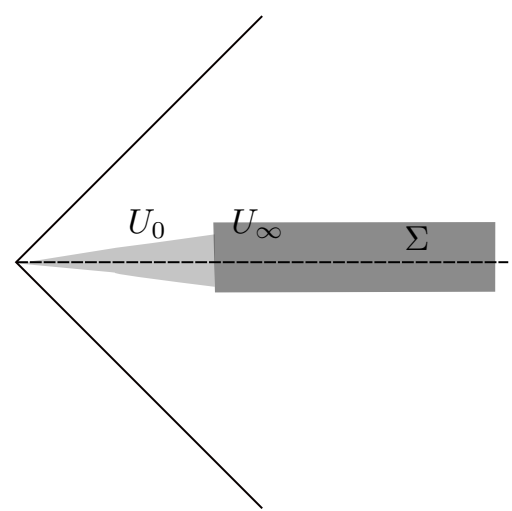

Fig. 4 The standard wedge

The standard wedge is

$$
M=\left\{\left(t, x_{1}, x^{\prime}\right) \in \mathbb{R}^{1+d}:|t|<x_{1}\right\}, \quad d s^{2}=-d t^{2}+d x_{1}^{2}+d x^{\prime 2} .
$$

We take again $\Sigma=M \cap\{t=0\}$. We take:

$$
U_{0}=\left\{|t|<\delta x_{1}, \quad t^{2}+x_{1}^{2}<1\right\}, U_{\infty}=\left\{|t|<\delta, 2<x_{1}\right\} .
$$

We check hypotheses $(\mathrm{H})$ over $U_{0}$ as above, replacing $1-r$ by $x_{1}$ and $\omega$ by $x^{\prime}$. Hypotheses $(\mathrm{H})$ over $U_{\infty}$ are immediate since $g$ is the Minkowski metric. Thus, $(\mathrm{H})$ is satisfied over $U_{0} \cup U_{\infty}$. Hypothesis (M) is again satisfied for $V=m^{2}$.

\subsubsection{Lightcones in Minkowski.}

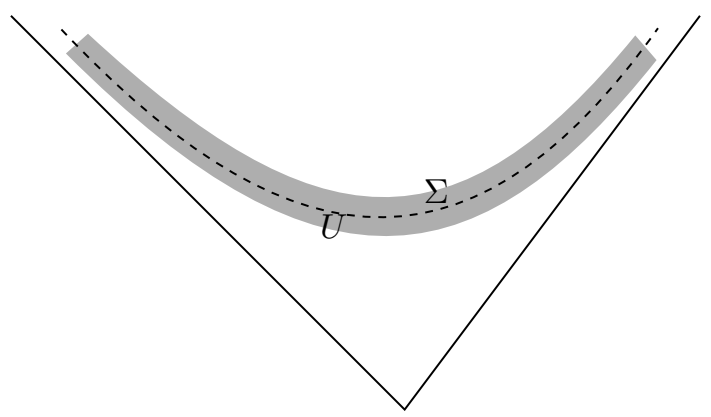

Fig.5 The future lightcone

The future lightcone is

$$
M=\left\{(t, x) \in \mathbb{R}^{1+d}: t>|x|\right\}, d s^{2}=-d t^{2}+d x^{2} .
$$

We choose $\Sigma=M \cap\left\{t^{2}-x^{2}=1\right\}$, use polar coordinates $x=r \omega$ and set

$$
r=\mathrm{e}^{-T} \operatorname{sh} X, t=\mathrm{e}^{-T} \operatorname{ch} X,
$$


so that

$$
\begin{array}{r}
M=\mathbb{R}_{T} \times \mathbb{R}_{X} \times \mathbb{S}_{\omega}^{d-1}, \Sigma=\{T=0\}, \\
d s^{2}=\mathrm{e}^{-2 T}\left(-d T^{2}+d X^{2}+\operatorname{sh}^{2} X d \omega^{2}\right) .
\end{array}
$$

We take $U=]-\delta, \delta\left[T_{T} \times \mathbb{R}_{X} \times \mathbb{S}_{\omega}^{d-1}\right.$ as neighborhood of $\Sigma$. As before it suffices to check hypotheses $(\mathrm{H})$ over $U \cap\{|X|>1\}$. We take $\tilde{c}(T, X)=\mathrm{e}^{-T}$ and choose the reference Riemannian metric

$$
\hat{g}=d T^{2}+d X^{2}+\mathrm{e}^{2|X|} d \omega^{2}
$$

which is of bounded geometry by Prop. 2.13. Then $\tilde{g}=-d T^{2}+d X^{2}+\operatorname{sh}^{2} X d \omega^{2}$ and hypotheses $(\mathrm{H})$ are clearly satisfied, as is hypothesis (M) for $V=m^{2}$.

\section{Pseudodifferential Calculus on manifolds of Bounded GeOmetry}

5.1. Introduction. In this section we recall the uniform pseudodifferential calculus on a manifold of bounded geometry, due to Kordyukov [Ko and Shubin [Sh1. This calculus generalizes for example the pseudodifferential calculus on a compact manifold and the uniform pseudodifferential calculus on $\mathbb{R}^{n}$. An important result for us is the generalization of Seeley's theorem [Se], originally proved on a compact manifold.

More precisely, if $A \in \Psi^{m}(M)$ is an elliptic pseudodifferential operator of order $m \geq 0$ on $M$, symmetric and strictly positive on $C_{0}^{\infty}(M)$, then $A$ has a unique selfadjoint extension, still denoted by $A$, with domain $H^{m}(M)$. Then Seeley's theorem asserts that $A^{z}$ is a holomorphic family of pseudodifferential operators of order $m \operatorname{Re} z$.

The extension of Seeley's theorem to pseudodifferential operators on manifolds of bounded geometry is due to ALNV, which we will closely follow.

Another important result proved in this section is Egorov's theorem. It is usually formulated as the fact that if $A$ is a pseudodifferential operator and $\mathcal{U}$ a Fourier integral operator then $B=\mathcal{U}^{-1} A \mathcal{U}$ is again a pseudodifferential operator. In our case we will take $\mathcal{U}=\mathcal{U}_{\epsilon}(t, s)$ equal to the evolution group generated by a smooth time-dependent family $\epsilon(t)$ of elliptic first order $\Psi$ DOs, with real principal symbol.

It will be convenient to consider also time-dependent pseudodifferential operators $A=A(t) \in C_{\mathrm{b}}^{\infty}\left(I ; \Psi^{m}(M)\right)$ for $I \subset \mathbb{R}$ an open interval. It turns out that the framework of ALNV is general enough to accommodate this extension without much additional work.

5.2. Symbol classes. In this subsection we recall well-known definitions about symbol classes.

5.2.1. Symbol classes on $\mathbb{R}^{n}$. Let $U \subset \mathbb{R}^{n}$ be an open set, equipped with the flat metric $\delta$ on $\mathbb{R}^{n}$.

we denote by $S^{m}\left(T^{*} U\right), m \in \mathbb{R}$, the space of $a \in C^{\infty}\left(U \times \mathbb{R}^{n}\right)$ such that

$$
\langle\xi\rangle^{-m+|\beta|} \partial_{x}^{\alpha} \partial_{\xi}^{\beta} a(x, \xi) \text { is bounded on } U \times \mathbb{R}^{n}, \forall \alpha, \beta \in \mathbb{N}^{n},
$$

equipped with its canonical seminorms $\|\cdot\|_{m, \alpha, \beta}$.

We set

$$
S^{-\infty}\left(T^{*} U\right):=\bigcap_{m \in \mathbb{R}} S^{m}\left(T^{*} U\right), \quad S^{\infty}\left(T^{*} U\right):=\bigcup_{m \in \mathbb{R}} S^{m}\left(T^{*} U\right),
$$

with their canonical Fréchet space topologies.

If $m \in \mathbb{R}$ and $a_{m-i} \in S^{m-i}\left(T^{*} U\right)$ we write

$$
a \simeq \sum_{i \in \mathbb{N}} a_{m-i}
$$


if for each $p \in \mathbb{N}$

$$
r_{p}(a):=a-\sum_{i=0}^{p} a_{m-i} \in S^{m-p-1}\left(T^{*} U\right) .
$$

It is well-known (see e.g. [Sh1, Sect. 3.3]) that if $a_{m-i} \in S^{m-i}\left(T^{*} U\right)$, there exists $a \in S^{m}\left(T^{*} U\right)$, unique modulo $S^{-\infty}\left(T^{*} U\right)$ such that $a \simeq \sum_{i \in \mathbb{N}} a_{m-i}$.

We denote by $S_{\mathrm{h}}^{m}\left(T^{*} U\right) \subset S^{m}\left(T^{*} U\right)$ the space of $a$ such that $a(x, \lambda \xi)=\lambda^{m} a(x, \xi)$, for $x \in U,|\xi| \geq C, C>0$.

We denote by $S_{\mathrm{ph}}^{m}\left(T^{*} U\right) \subset S^{m}\left(T^{*} U\right)$ the space of $a$ such that $a \simeq \sum_{i \in \mathbb{N}} a_{m-i}$ for a sequence $a_{m-i} \in S_{\mathrm{h}}^{m-i}\left(T^{*} U\right)$.

Following [ALNV], we equip $S_{\mathrm{ph}}^{m}\left(T^{*} U\right)$ with the topology defined by the seminorms of $a_{m-i}$ in $S^{m-i}\left(T^{*} U\right)$ and $r_{p}(a)$ in $S^{m-p-1}\left(T^{*} U\right)$, (see (5.1)). This topology is strictly stronger than the topology induced by $S^{m}\left(T^{*} U\right)$.

The space $S_{\mathrm{ph}}^{m}\left(T^{*} U\right) / S_{\mathrm{ph}}^{m-1}\left(T^{*} U\right)$ is isomorphic to $S_{\mathrm{h}}^{m}\left(T^{*} U\right)$, and the image of $a$ under the quotient map is called the principal part of $a$ and denoted by $a_{\mathrm{pr}}$.

Finally we note that if $U=B_{n}(0,1)$ (more generally if $U$ is relatively compact with smooth boundary), there exists a continuous extension map $E: S^{m}\left(T^{*} U\right) \rightarrow$ $S^{m}\left(T^{*} \mathbb{R}^{n}\right)$ such that $\left.E a\right|_{T^{*} U}=a$. Moreover $E$ maps $S_{\mathrm{ph}}^{m}\left(T^{*} U\right)$ into $S_{\mathrm{ph}}^{m}\left(T^{*} \mathbb{R}^{n}\right)$ and is continuous for the topologies of $S_{\mathrm{ph}}^{m}\left(T^{*} U\right)$ and $S_{\mathrm{ph}}^{m}\left(T^{*} \mathbb{R}^{n}\right)$, which means that all the maps

are continuous.

$$
a \mapsto(E a)_{m-i}, a \mapsto r_{p}(E a),
$$

5.2.2. Time-dependent symbol classes on $\mathbb{R}^{n}$. let $I \subset \mathbb{R}$ an open interval. We will also need to consider time-dependent symbols $a(t, x, \xi) \in C^{\infty}\left(I \times T^{*} U\right)$.

The space $C_{\mathrm{b}}^{\infty}\left(I ; S^{m}\left(T^{*} U\right)\right)$ is naturally defined as the space of $a \in C^{\infty}\left(I \times T^{*} U\right)$ such that

$$
\langle\xi\rangle^{-m+|\beta|} \partial_{t}^{\gamma} \partial_{x}^{\alpha} \partial_{\xi}^{\beta} a(x, \xi) \text { is bounded on } I \times U \times \mathbb{R}^{n}, \forall \alpha, \beta \in \mathbb{N}^{n}, \gamma \in \mathbb{N},
$$

equipped with its canonical seminorms $\|\cdot\|_{m, \alpha, \beta, \gamma}$. The notation $a \sim \sum_{i} a_{m-i}$ and the subspaces $C_{\mathrm{b}}^{\infty}\left(I ; S_{\mathrm{ph}}^{m}\left(T^{*} U\right)\right)$ are defined accordingly, by requiring uniform estimates on $I$ of all time derivatives.

\subsubsection{Symbol classes on $M$.}

Definition 5.1. We denote by $S^{m}\left(T^{*} M\right)$ the space of $a \in C^{\infty}\left(T^{*} M\right)$ such that for each $x \in M, a_{x}:=\left(\psi_{x}^{-1}\right)^{*} a \in S^{m}\left(T^{*} B_{n}(0,1)\right)$ and the family $\left\{a_{x}\right\}_{x \in M}$ is bounded in $S^{m}\left(T^{*} B_{n}(0,1)\right)$. We equip $S^{m}\left(T^{*} M\right)$ with the seminorms

$$
\|a\|_{m, \alpha, \beta}=\sup _{x \in M}\left\|a_{x}\right\|_{m, \alpha, \beta} .
$$

Similarly we denote by $S_{\mathrm{ph}}^{m}\left(T^{*} M\right)$ the space of $a \in S^{m}\left(T^{*} M\right)$ such that for each $x \in$ $M, a_{x} \in S_{\mathrm{ph}}^{m}\left(T^{*} B_{n}(0,1)\right)$ and the family $\left\{a_{x}\right\}_{x \in M}$ is bounded in $S_{\mathrm{ph}}^{m}\left(T^{*} B_{n}(0,1)\right)$. We equip $S_{\mathrm{ph}}^{m}\left(T^{*} M\right)$ with the seminorms

$$
\|a\|_{m, i, p, \alpha, \beta}=\sup _{x \in M}\left\|a_{x}\right\|_{m, i, p, \alpha, \beta} .
$$

where $\|\cdot\|_{m, i, p, \alpha, \beta}$ are the seminorms defining the topology of $S_{\mathrm{ph}}^{m}\left(T^{*} B_{n}(0,1)\right)$.

It is easy to see that the definition of $S^{m}\left(T^{*} M\right), S_{\mathrm{ph}}^{m}\left(T^{*} M\right)$ and their Fréchet space topologies are independent on the choice of the $\left\{U_{x}, \psi_{x}\right\}_{x \in M}$, with the above properties.

The notation $a \simeq \sum_{i \in \mathbb{N}} a_{m-i}$ for $a_{m-i} \in S_{\mathrm{ph}}^{m-i}\left(T^{*} M\right)$ is defined as before. If $a \in S_{\mathrm{ph}}^{m}\left(T^{*} M\right)$, we denote again by $a_{\mathrm{pr}}$ the image of $a$ in $S_{\mathrm{ph}}^{m}\left(T^{*} M\right) / S_{\mathrm{ph}}^{m-1}\left(T^{*} M\right)$. 
If $I \subset \mathbb{R}$ is an open interval, the spaces $C_{\mathrm{b}}^{\infty}\left(I ; S^{m}\left(T^{*} M\right)\right)$ and $C_{\mathrm{b}}^{\infty}\left(I ; S_{\mathrm{ph}}^{m}\left(T^{*} M\right)\right)$ are defined as in 5.2 .2

5.3. Pseudodifferential operators. We now recall standard facts about the associated pseudodifferential operators, see [Ko, Sh1, ALNV].

5.3.1. Pseudodifferential operators on $\mathbb{R}^{n}$. If $a \in S^{m}\left(T^{*} \mathbb{R}^{n}\right)$, we denote by $\mathrm{Op}^{\mathrm{w}}(a)$ its Weyl quantization, defined by

$$
\mathrm{Op}^{\mathrm{w}}(a) u(x)=(2 \pi)^{-n} \int \mathrm{e}^{\mathrm{i}(x-y) \cdot \xi} a\left(\frac{x+y}{2}, \xi\right) u(y) d y d \xi
$$

We recall the following well-known properties:

(1) $\mathrm{Op}^{\mathrm{w}}(a): C_{0}^{\infty}\left(\mathbb{R}^{n}\right) \rightarrow \mathcal{E}^{\prime}\left(\mathbb{R}^{n}\right)$ is continuous,

(2) Op : $S^{m}\left(T^{*} \mathbb{R}^{n}\right) \rightarrow \bigcap_{s \in \mathbb{R}} B\left(H^{s}\left(\mathbb{R}^{n}\right), H^{s-m}\left(\mathbb{R}^{n}\right)\right)$ is continuous, where $H^{s}\left(\mathbb{R}^{n}\right)$ is the Sobolev space of order $s$ on $\mathbb{R}^{n}$.

(3) there exists a bilinear continuous map

$$
S^{\infty}\left(T^{*} \mathbb{R}^{n}\right) \times S^{\infty}\left(T^{*} \mathbb{R}^{n}\right) \ni(a, b) \mapsto a \sharp b \in S^{\infty}\left(T^{*} \mathbb{R}^{n}\right)
$$

such that $\mathrm{Op}^{\mathrm{w}}(a) \mathrm{Op}^{\mathrm{w}}(b)=\mathrm{Op}^{\mathrm{w}}(a \sharp b)$.

5.3.2. Time-dependent pseudodifferential operators on $\mathbb{R}^{n}$. If $I \subset \mathbb{R}$ is an open interval and $a=a(t) \in C_{\mathrm{b}}^{\infty}\left(I ; S^{m}\left(T^{*} M\right)\right)$ we can consider the time-dependent pseudodifferential operator $\mathrm{Op}^{\mathrm{w}}(a(t))$. We have

(1) $\mathrm{Op}^{\mathrm{w}}(a(t)): C_{\mathrm{b}}^{\infty}\left(I ; C_{0}^{\infty}\left(\mathbb{R}^{n}\right)\right) \rightarrow C_{\mathrm{b}}^{\infty}\left(I ; \mathcal{E}^{\prime}\left(\mathbb{R}^{n}\right)\right)$ is continuous,

(2) Op : $C_{\mathrm{b}}^{\infty}\left(I ; S^{m}\left(T^{*} \mathbb{R}^{n}\right)\right) \rightarrow \bigcap_{r, s \in \mathbb{R}} B\left(H^{r}\left(I ; H^{s}\left(\mathbb{R}^{n}\right)\right), H^{r}\left(I ; H^{s-m}\left(\mathbb{R}^{n}\right)\right)\right)$ is continuous, where $H^{r}\left(I ; H^{s}\left(\mathbb{R}^{n}\right)\right)$ is the Sobolev space of bi-order $r, s$ on $I \times \mathbb{R}^{n}$.

5.3.3. Quantization maps. We now recall the quantization procedure on a manifold of bounded geometry. Let $\left\{U_{i}, \psi_{i}\right\}_{i \in \mathbb{N}}$ be a good chart covering of $M$ and

$$
\sum_{i \in \mathbb{N}} \chi_{i}^{2}=\mathbb{1}
$$

a subordinate good partition of unity, see Subsect. 2.2. Let

$$
\left(\psi_{i}^{-1}\right)^{*} d g=: m_{i} d x
$$

so that $\left\{m_{i}\right\}_{i \in \mathbb{N}}$ is bounded in $C_{\mathrm{b}}^{\infty}\left(B_{n}(0,1)\right)$. We set also:

$$
\begin{aligned}
T_{i}: \quad & L^{2}\left(U_{i}, d g\right) \rightarrow L^{2}\left(B_{n}(0,1), d x\right), \\
& u \mapsto m_{i}^{\frac{1}{2}}\left(\psi_{i}^{-1}\right)^{*} u,
\end{aligned}
$$

so that $T_{i}: L^{2}\left(U_{i}, d g\right) \rightarrow L^{2}\left(B_{n}(0,1), d x\right)$ is unitary.

Definition 5.2. Let $a=a(t) \in C_{\mathrm{b}}^{\infty}\left(I ; S^{m}\left(T^{*} M\right)\right)$. We set

$$
\mathrm{Op}(a):=\sum_{i \in \mathbb{N}} \chi_{i} T_{i}^{*} \circ \mathrm{Op}^{\mathrm{w}}\left(E a_{i}\right) \circ T_{i} \chi_{i}
$$

where $a_{i}=a_{x_{i}}$ (see Def. 5.1), and $E$ is the extension map (see Subsect. 5.2). Clearly $\mathrm{Op}(a): C_{\mathrm{b}}^{\infty}\left(I ; C_{0}^{\infty}(\bar{M})\right) \rightarrow C_{\mathrm{b}}^{\infty}\left(I ; \mathcal{E}^{\prime}(M)\right)$ is continuous.

Such a map Op obtained from a good chart covering and partition of unity will be called a good quantization map.

Note that $\operatorname{Op}(1)=\mathbb{1}$, and that $\operatorname{Op}(a)^{*}(t)=\mathrm{Op}(\bar{a})(t)$ on $C_{0}^{\infty}(M)$, where $A^{*}$ is the adjoint of $A$ for the scalar product

$$
(u \mid v)_{M}=\int_{M} \bar{u} v d \operatorname{vol}_{g} .
$$


Note that if $A(t) \in C_{\mathrm{b}}^{\infty}\left(I ; \mathrm{Op}\left(S^{\infty}\left(T^{*} M\right)\right)\right)$, then its distributional kernel $A(t, x, y)$ is supported in

$$
\{(x, y) \in M \times M: d(x, y) \leq C\},
$$

for some $C>0$, where $d$ is the geodesic distance on $M$. It follows that $\operatorname{Op}(a)$ : $C_{0}^{\infty}(M) \rightarrow C_{0}^{\infty}(M)$, hence $\mathrm{Op}(a) \circ \mathrm{Op}(b)$ is well defined. However because of the above support property $\operatorname{Op}\left(S^{\infty}\left(T^{*} M\right)\right)$ is not stable under composition. To obtain an algebra of operators, it is necessary to add to $\operatorname{Op}\left(S^{\infty}\left(T^{*} M\right)\right)$ an ideal of smoothing operators, which we introduce below.

The Sobolev spaces $H^{s}(M, g)$ defined in 2.3 .3 will be simply denoted by $H^{s}(M)$. We will set:

$$
H^{\infty}(M)=\bigcap_{m \in \mathbb{Z}} H^{m}(M), H^{-\infty}(M)=\bigcup_{m \in \mathbb{Z}} H^{m}(M),
$$

equipped with their natural topologies.

Definition 5.3. We set:

$$
\mathcal{W}^{-\infty}(M):=\bigcap_{m \in \mathbb{N}} B\left(H^{-m}(M), H^{m}(M)\right),
$$

equipped with its natural topology given by the seminorms

$$
\|A\|_{m}=\left\|\left(-\Delta_{g}+1\right)^{m / 2} A\left(-\Delta_{g}+1\right)^{m / 2}\right\|_{B\left(L^{2}(M)\right)} .
$$

Similarly we equip

$$
C_{\mathrm{b}}^{\infty}\left(I ; \mathcal{W}^{-\infty}(M)\right)
$$

with the topology given by the seminorms

$$
\|A\|_{m, p}=\sup _{t \in I, k \leq p}\left\|\partial_{t}^{k} A(t)\right\|_{m} .
$$

The following result, showing the independence modulo $C_{\mathrm{b}}^{\infty}\left(I ; W^{-\infty}(M)\right)$ of $\mathrm{Op}\left(C_{\mathrm{b}}^{\infty}\left(I ; S^{\infty}\left(T^{*} M\right)\right)\right)$ of the above choices of $\left\{U_{i}, \psi_{i}, \chi_{i}\right\}$, is easy to prove.

Proposition 5.4. Let $\mathrm{Op}^{\prime}$ another good quantization map. Then

$$
\mathrm{Op}-\mathrm{Op}^{\prime}: C_{\mathrm{b}}^{\infty}\left(I ; S^{\infty}\left(T^{*} M\right)\right) \rightarrow C_{\mathrm{b}}^{\infty}\left(I ; \mathcal{W}^{-\infty}(M)\right) .
$$

is continuous.

5.3.4. The axioms of a Weyl algebra. In ALNV, a set of abstract axioms was introduced, with the aim of defining pseudodifferential operators on a manifold in a very general framework. The main result of [ALNV] is the extension of Seeley's theorem [Se]. We will now check the abstract axioms of [ALNV, Sect. 1] in our situation. Namely, we need to specify a tuple $\left(\cup_{k \geq 1} \mathcal{W}_{k}^{-\infty}, \mathcal{H}, q, \sharp\right)$ that satisfies the following properties (we refer the reader to [ALNV] 1.2] for the precise formulation in the general case):

Axiom (i): the LF-algebra and the Hilbert space: One requires that $\mathcal{H}$ is a Hilbert space and $\mathcal{W}^{-\infty}=\cup_{k \geq 1} \mathcal{W}_{k}^{-\infty}$ is a LF-algebr25 continuously embedded in $B(\mathcal{H})$ and such that the adjoint operation ${ }^{*}$ maps $\mathcal{W}^{-\infty} \rightarrow \mathcal{W}^{-\infty}$ continuously. We choose $\mathcal{H}=L^{2}\left(I ; L^{2}(M, d g)\right)$ and $\mathcal{W}^{-\infty}=\mathcal{W}_{k}^{-\infty}=C_{\mathrm{b}}^{\infty}\left(I ; \mathcal{W}^{-\infty}(M)\right)$. The LF-algebra properties are immediate. Furthermore, we have indeed $C_{\mathrm{b}}^{\infty}\left(I ; \mathcal{W}^{-\infty}(M)\right) \subset B(\mathcal{H})$ and

$$
\left(C_{\mathrm{b}}^{\infty}\left(I ; \mathcal{W}^{-\infty}(M)\right)\right)^{*}=C_{\mathrm{b}}^{\infty}\left(I ; \mathcal{W}^{-\infty}(M)\right) .
$$

Axiom (ii): existence of an injective, self-adjoint operator in $\mathcal{W}^{-\infty}$ : We choose the (time-independent) operator $R=\mathrm{e}^{-\left(\Delta_{g}+1\right)}$. Clearly $R=R^{*} \in C_{\mathrm{b}}^{\infty}\left(I ; \mathcal{W}^{-\infty}(M)\right)$.

\footnotetext{
${ }^{5}$ This means that $\mathcal{W}^{-\infty}$ is a strict inductive limit of Fréchet spaces and is endowed with an algebra structure with some additional grading and continuity properties, see [ALNV] 1.2].
} 
Axiom (iii): quantization map q: We choose $q(a):=\mathrm{Op}(a)$. One needs to check in our case that

$$
\begin{aligned}
& \mathrm{Op}(a): L^{2}\left(I ; H^{-\infty}(M)\right) \rightarrow L^{2}\left(I ; H^{-\infty}(M)\right), \\
& \text { Op : } C_{\mathrm{b}}^{\infty}\left(I ; S^{-\infty}\left(T^{*} M\right)\right) \rightarrow C_{\mathrm{b}}^{\infty}\left(I ; \mathcal{W}^{-\infty}(M)\right),
\end{aligned}
$$

which is straightforward from the properties of Op already listed.

Axiom (iv): It is easy to check using for example the norm given in (2.7) that $\mathrm{Op}(a) \in C_{\mathrm{b}}^{\infty}\left(I ; B\left(H^{s}(M), H^{s-m}(M)\right)\right)$ for $a \in C_{\mathrm{b}}^{\infty}\left(I ; S^{m}\left(T^{*} M\right)\right)$. This implies that

$$
\mathrm{Op}\left(C_{\mathrm{b}}^{\infty}\left(I ; S^{\infty}\left(T^{*} M\right)\right)\right) C_{\mathrm{b}}^{\infty}\left(I ; \mathcal{W}^{-\infty}(M)\right) \subset C_{\mathrm{b}}^{\infty}\left(I ; \mathcal{W}^{-\infty}(M)\right),
$$

which is in our setting the required property of the quantization map $q$.

Axiom (v): existence of a symbolic calculus: from the symbolic calculus in $\mathrm{Op}^{\mathrm{w}}\left(S^{m}\left(T^{*} \mathbb{R}^{n}\right)\right)$ we obtain the existence of a bilinear map

$$
(a, b) \mapsto a \sharp b \text { defined on } C_{\mathrm{b}}^{\infty}\left(I ; S^{\infty}\left(T^{*} M\right)\right)
$$

such that

$$
\mathrm{Op}(a) \mathrm{Op}(b)-\mathrm{Op}(a \sharp b) \in C_{\mathrm{b}}^{\infty}\left(I ; \mathcal{W}^{-\infty}(M)\right), \text { for } a, b \in C_{\mathrm{b}}^{\infty}\left(I ; S^{\infty}\left(T^{*} M\right)\right) .
$$

Concretely we have

$$
a \sharp b=\sum_{i \in \mathbb{N}} \chi_{i}^{2} \psi_{i}^{*}\left(a_{i} \sharp b_{i}\right),
$$

where $a_{i} \sharp b_{i}$ is recalled at the beginning of Subsect. 5.2 . The fact that $a \sharp b$ as an asymptotic expansion in terms of homogeneous bi-differential operators follows from the analogous property of the symbolic calculus on $\mathbb{R}^{n}$.

Axiom (vi): boundedness of $\Psi D O s$ : from the analogous property on $\mathbb{R}^{n}$ we easily obtain that

$$
\text { Op : } C_{\mathrm{b}}^{\infty}\left(I ; S^{0}\left(T^{*} M\right)\right) \rightarrow B\left(L^{2}\left(I ; L^{2}(M)\right)\right) \text { is continuous. }
$$

Axiom (vii): One requires that the map

$$
C_{\mathrm{b}}^{\infty}\left(I ; S^{m}\left(T^{*} M\right)\right) \times C_{\mathrm{b}}^{\infty}\left(I ; \mathcal{W}^{-\infty}(M)\right) \ni(a, T) \mapsto \mathrm{Op}(a) \circ T \in C_{\mathrm{b}}^{\infty}\left(I ; \mathcal{W}^{-\infty}(M)\right)
$$

is continuous. This follows from axiom (vi) in our situation.

Two further important conditions are introduced in [ALNV].

The first condition, called condition $(\sigma)$ in [ALNV] amounts to the property that if $a \in C^{\infty}\left(I ; S_{\mathrm{ph}}^{m}\left(T^{*} M\right)\right)$ and $\operatorname{Op}(a) \in C_{\mathrm{b}}^{\infty}\left(I ; \mathcal{W}^{-\infty}(M)\right)$, then $a$ belongs to $C_{\mathrm{b}}^{\infty}\left(I ; S^{-\infty}\left(T^{*} M\right)\right)$. In our case we deduce from the properties of the $\Psi$ DO calculus on $\mathbb{R}^{n}$ that the sequence $\left\{a_{i}\right\}_{i \in \mathbb{N}}$ is uniformly bounded in $C_{\mathrm{b}}^{\infty}\left(I ; S^{-\infty}\left(T^{*} B_{n}(0,1)\right)\right)$, which implies that $a \in C_{\mathrm{b}}^{\infty}\left(I ; S^{-\infty}\left(T^{*} M\right)\right)$.

The second condition, called condition $(\psi)$ in [ALNV], is the spectral invariance of the algebra $11+C_{\mathrm{b}}^{\infty}\left(I ; \mathcal{W}^{-\infty}(M)\right)$. This condition is stated and proved in the lemma below.

Lemma 5.5. Let $R_{-\infty} \in C_{\mathrm{b}}^{\infty}\left(I ; \mathcal{W}^{-\infty}(M)\right)$ such that $\mathbb{1}-R_{-\infty}$ is invertible in $B\left(L^{2}\left(I ; L^{2}(M)\right)\right)$. Then

$$
\left(\mathbb{1}-R_{-\infty}\right)^{-1}=\mathbb{1}-R_{1,-\infty} \text { for } R_{1,-\infty} \in C_{\mathrm{b}}^{\infty}\left(I ; \mathcal{W}^{-\infty}(M)\right) .
$$

Proof. On $L^{2}\left(I ; L^{2}(M)\right) \sim \int_{I}^{\oplus} L^{2}(M) d t$ we have:

$$
\mathbb{1}-R_{-\infty}=\int_{I}^{\oplus} \mathbb{1}-R_{-\infty}(t) d t
$$

hence

$$
\left(\mathbb{1}-R_{-\infty}\right)^{-1}=\int_{I}^{\oplus}\left(\mathbb{1}-R_{-\infty}(t)\right)^{-1} d t
$$


and

$$
\begin{aligned}
\left\|\left(\mathbb{1}-R_{-\infty}\right)^{-1}\right\|_{B\left(L^{2}\left(I ; L^{2}(M)\right)\right)} & =\operatorname{ess}_{\sup _{t \in I}}\left\|\left(\mathbb{1}-R_{-\infty}(t)\right)^{-1}\right\|_{B\left(L^{2}(M)\right)} \\
& =\sup _{t \in I}\left\|\left(\mathbb{1}-R_{-\infty}(t)\right)^{-1}\right\|_{B\left(L^{2}(M)\right)},
\end{aligned}
$$

since

$$
I \ni t \mapsto\left(\mathbb{1}-R_{-\infty}(t)\right)^{-1} \in B\left(L^{2}(M)\right)
$$

is norm continuous. We have

$$
\left(\mathbb{1}-R_{-\infty}(t)\right)^{-1}=\mathbb{1}+R_{-\infty}(t)+R_{-\infty}(t)\left(\mathbb{1}-R_{-\infty}(t)\right)^{-1} R_{-\infty}(t) .
$$

Since $\left(1-R_{-\infty}(t)\right)^{-1} \in B\left(L^{2}(M)\right)$ and $R_{-\infty}(t) \in \mathcal{W}^{-\infty}(M)$, we see that $R_{-\infty}(t)(1-$ $\left.R_{-\infty}(t)\right)^{-1} R_{-\infty}(t) \in \mathcal{W}^{-\infty}(M)$. To prove that $R_{1,-\infty} \in C_{\mathrm{b}}^{\infty}\left(I ; \mathcal{W}^{-\infty}(M)\right)$ we differentiate (5.3) w.r.t. $t$ using the Leibniz rule and the identity

$$
\partial_{t}\left(\mathbb{1}-R_{-\infty}(t)\right)^{-1}=\left(\mathbb{1}-R_{-\infty}(t)\right)^{-1} \partial_{t} R_{-\infty}(t)\left(\mathbb{1}-R_{-\infty}(t)\right)^{-1} .
$$

5.3.5. Time-dependent pseudodifferential operators on $M$. We can now define classes of time-dependent pseudodifferential operators on $M$, by applying the abstract framework of [ALNV, Sect. 1]. We will only consider classical pseudodifferential operators, i.e. operators obtained from poly-homogeneous symbols.

Definition 5.6. We set for $m \in \mathbb{R}$ :

$$
C_{\mathrm{b}}^{\infty}\left(I ; \Psi^{m}(M)\right):=\operatorname{Op}\left(C_{\mathrm{b}}^{\infty}\left(I ; S_{\mathrm{ph}}^{m}\left(T^{*} M\right)\right)\right)+C_{\mathrm{b}}^{\infty}\left(I ; \mathcal{W}^{-\infty}(M)\right) .
$$

Remark 5.7. An element of $C_{\mathrm{b}}^{\infty}\left(I ; \Psi^{m}(M)\right)$ will usually be denoted by $A$, while $A(t)$ for $t \in I$ will be an element of $\Psi^{m}(M)$. Writing for example $L^{2}\left(I ; L^{2}(M)\right.$ ) as $\int_{I}^{\oplus} L^{2}(M) d t$, we have

$$
A=\int_{I}^{\oplus} A(t) d t .
$$

Note that $C_{\mathrm{b}}^{\infty}\left(I ; \Psi^{-\infty}(M)\right)=C_{\mathrm{b}}^{\infty}\left(I ; \mathcal{W}^{-\infty}(M)\right)$. If necessary we equip the space $C_{\mathrm{b}}^{\infty}\left(I ; \Psi^{m}(M)\right)$ with the quotient topology obtained from the map

$$
C_{\mathrm{b}}^{\infty}\left(I ; S_{\mathrm{ph}}^{m}\left(T^{*} M\right)\right) \times C_{\mathrm{b}}^{\infty}\left(I ; \mathcal{W}^{-\infty}(M)\right) \in(a, R) \mapsto \mathrm{Op}(a)+R \in C_{\mathrm{b}}^{\infty}\left(I ; \Psi^{m}(M)\right) .
$$

It follows that the injection:

$$
C_{\mathrm{b}}^{\infty}\left(I ; \Psi^{m}(M)\right) \rightarrow \bigcap_{s \in \mathbb{R}} C_{\mathrm{b}}^{\infty}\left(I ; B\left(H^{s}(M), H^{s-m}(M)\right)\right)
$$

is continuous.

Definition 5.8. Let $A=\mathrm{Op}(a)+R_{-\infty} \in C_{\mathrm{b}}^{\infty}\left(I ; \Psi^{m}(M)\right)$. We denote by $\sigma_{\mathrm{pr}}(A) \in$ $C_{\mathrm{b}}^{\infty}\left(I ; S_{\mathrm{h}}^{m}\left(T^{*} M\right)\right)$ the principal symbol of $A$ defined as

$$
\sigma_{\mathrm{pr}}(A):=[a] \in C_{\mathrm{b}}^{\infty}\left(I ; S_{\mathrm{ph}}^{m}\left(T^{*} M\right)\right) / C_{\mathrm{b}}^{\infty}\left(I ; S_{\mathrm{ph}}^{m-1}\left(T^{*} M\right)\right) .
$$

By property $(\sigma)$ and Prop. $5.4 \sigma_{\mathrm{pr}}(A)$ is independent on the decomposition of $A$ as $\mathrm{Op}(a)+R_{-\infty}$ and on the choice of the good quantization map Op.

Definition 5.9. $A \in C_{\mathrm{b}}^{\infty}\left(I ; \Psi^{m}(M)\right)$ is elliptic if there exists $C>0$ such that

$$
\left|\sigma_{\mathrm{pr}}(A)(t, x, \xi)\right| \geq C\left(\xi \cdot g^{-1}(x) \xi\right)^{m / 2}, t \in I,(x, \xi) \in T^{*} M .
$$

The main property of elliptic operators is that they admit parametrices, i.e. inverses modulo $C_{\mathrm{b}}^{\infty}\left(I ; \mathcal{W}^{-\infty}(M)\right)$.

Proposition 5.10. Let $A \in C_{\mathrm{b}}^{\infty}\left(I ; \Psi^{m}(M)\right)$ be elliptic. Then there exists $B \in$ $C_{\mathrm{b}}^{\infty}\left(I ; \Psi^{-m}(M)\right)$, unique modulo $C_{\mathrm{b}}^{\infty}\left(I ; \mathcal{W}^{-\infty}(M)\right)$ such that

$$
A B-\mathbb{1} \in C_{\mathrm{b}}^{\infty}\left(I ; \mathcal{W}^{-\infty}(M)\right), \quad B A-\mathbb{1} \in C_{\mathrm{b}}^{\infty}\left(I ;\left(\mathcal{W}^{-\infty}(M)\right) .\right.
$$

Such an operator $B$ is called a parametrix of $A$ and denoted by $A^{(-1)}$. 
Proof. The proof given in [Ko, Thm. 3.3] or [Sh2, Prop. 3.4] extends immediately to the time-dependent situation.

We recall that the notation $a \sim b$ for $a, b$ are two selfadjoint operators on a Hilbert space $\mathcal{H}$ is defined in Subsect. 1.4

Proposition 5.11. Let $A \in C_{\mathrm{b}}^{\infty}\left(I ; \Psi^{m}(M)\right), m \geq 0$ be elliptic such that $A(t)$ is symmetric on $H^{\infty}(M)$ for all $t \in I$. Then

(1) $A(t)$ is essentially selfadjoint on $H^{\infty}(M)$ and

$$
\operatorname{Dom} A^{\mathrm{cl}}(t)=H^{m}(M) \text {. }
$$

(2) If in addition $\sigma_{\mathrm{pr}}(A)(t, x, \xi) \geq c\left(\xi \cdot g^{-1}(x) \xi\right)^{m / 2}$ for some $c>0$, then $A^{\mathrm{cl}}(t)$ is bounded below, uniformly for $t \in I$. Moreover there exists $R \in C_{\mathrm{b}}^{\infty}\left(I ;\left(\mathcal{W}^{-\infty}(M)\right)\right.$ such that

$$
A(t)+R_{-\infty}(t) \sim\left(-\Delta_{g}+1\right)^{m / 2}, \text { uniformly for } t \in I .
$$

(3) $A$ (considered as a linear operator on $L^{2}\left(I ; L^{2}(M)\right)$ ) is essentially selfadjoint on $L^{2}\left(I ; H^{\infty}(M)\right)$ and

$$
\operatorname{Dom} A^{\mathrm{cl}}=L^{2}\left(I ; H^{m}(M)\right) .
$$

Proof. statement (1) follows from [ALNV] Prop. 2.2] and the alternative characterization of Sobolev spaces given in [ALNV, Sect.3]. To prove (2) we may assume that $A=\operatorname{Op}(a)$ since $\mathcal{W}^{-\infty}(M) \subset B\left(L^{2}(M)\right)$. Then

$$
A(t)=\sum_{i \in \mathbb{N}} \chi_{i} T_{i}^{*} A_{i}(t) T_{i} \chi_{i}
$$

where $\left\{A_{i}\right\}_{i \in \mathbb{N}}$ is a bounded family in $\operatorname{Op}^{\mathrm{w}}\left(C_{\mathrm{b}}^{\infty}\left(I ; S^{m}\left(T^{*} \mathbb{R}^{n}\right)\right)\right)$ such that

$$
\sigma_{\mathrm{pr}}\left(A_{i}\right)(t, x, \xi) \geq c|\xi|^{m} \text {, uniformly for } i \in \mathbb{N}, t \in I .
$$

From the $\Psi D O$ calculus on $\mathbb{R}^{n}$ we deduce that $A_{i}(t) \geq c^{\prime} \mathbb{1}$ uniformly in $i \in \mathbb{N}$ and which shows that $A(t)$ is bounded below uniformly in $t \in I$. This also implies that for $c \gg 1$ one has $A(t)+c \sim\left(-\Delta_{g}+1\right)^{m / 2}$. By functional calculus we can find $\chi \in C_{0}^{\infty}(\mathbb{R})$ such that $A(t)+\chi(A(t)) \sim A(t)+c$. By elliptic regularity we know that $\chi(A) \in C_{\mathrm{b}}^{\infty}\left(I ; \mathcal{W}^{-\infty}(M)\right)$, which completes the proof of (2). (3) follows from (1).

We now state the main result of this subsection, which follows directly from ALNV, for the simpler case of real powers.

Theorem 5.12. Let $A \in C_{\mathrm{b}}^{\infty}\left(I ; \Psi^{m}(M)\right)$ be elliptic, selfadjoint with $A(t) \geq c \mathbb{1}$ for $c>0, t \in I$. Then $A^{s} \in C_{\mathrm{b}}^{\infty}\left(I ; \Psi^{m s}(M)\right)$ for any $s \in \mathbb{R}$ and

$$
\sigma_{\mathrm{pr}}\left(A^{s}\right)(t)=\sigma_{\mathrm{pr}}(A(t))^{s} \text {. }
$$

Proof. We consider $A$ as a selfadjoint operator on $L^{2}\left(I ; L^{2}(M)\right)$ and apply ALNV, Thm. 8.9], noting that $A^{s}(t)=A(t)^{s}$.

The following lemma will be used in Subsect. 7.4 .

Lemma 5.13. Let $A \in \Psi^{\infty}(M)$ such that $A: \mathcal{E}^{\prime}(M) \rightarrow C^{\infty}(M)$. Then $A \in$ $\mathcal{W}^{-\infty}(M)$.

Proof. We can assume that $A=\mathrm{Op}(a)$ for $a \in S_{\mathrm{ph}}^{m}\left(T^{*} M\right)$, i.e (see Def. 5.2 ):

$$
A=\sum_{i \in \mathbb{N}} \chi_{i} T_{i}^{*} \circ \mathrm{Op}^{\mathrm{w}}\left(E a_{i}\right) \circ T_{i} \chi_{i},
$$

where $\left\{E a_{i}\right\}_{i \in \mathbb{N}}$ is bounded in $S_{\mathrm{ph}}^{m}\left(T^{*} \mathbb{R}^{n}\right)$. We can fix cutoff functions $\tilde{\chi}_{i}$ such that $T_{i} \chi_{i}=\tilde{\chi}_{i} T_{i} \chi_{i},\left\{\tilde{\chi}_{i}\right\}_{i \in \mathbb{N}}$ is bounded in $C_{0}^{\infty}(B(0,1))$ and define $b_{i}$ by $\tilde{\chi}_{i} \circ \mathrm{Op}^{\mathrm{w}}\left(E a_{i}\right) \circ$ 
$\tilde{\chi}_{i}=\mathrm{Op}^{\mathrm{w}}\left(b_{i}\right)$. The family $\left\{b_{i}\right\}_{i \in \mathbb{N}}$ is bounded in $S_{\mathrm{ph}}^{m}\left(T^{*} \mathbb{R}^{n}\right)$ hence for each $p \in \mathbb{N}$ one has:

$$
b_{i}=\sum_{k=0}^{p} b_{i, m-k}+r_{i, p},
$$

where $\left\{b_{i, m-k}\right\}_{i \in \mathbb{N}}$, resp. $\left\{r_{i, p}\right\}_{i \in \mathbb{N}}$ is bounded in $S_{\mathrm{h}}^{m-k}\left(T^{*} \mathbb{R}^{n}\right)$ resp. $S^{m-p-1}\left(T^{*} \mathbb{R}^{n}\right)$. Since $A: \mathcal{E}^{\prime}(M) \rightarrow C^{\infty}(M)$ it follows that $\mathrm{Op}^{\mathrm{w}}\left(b_{i}\right): L^{2}\left(\mathbb{R}^{n}\right) \rightarrow H^{-m+k}\left(\mathbb{R}^{n}\right)$ for any $k \in \mathbb{N}$. Taking $k=1$ we obtain that $\operatorname{Op}^{\mathrm{w}}\left(b_{i, m}\right): L^{2}\left(\mathbb{R}^{n}\right) \rightarrow H^{-m+1}\left(\mathbb{R}^{n}\right)$, hence $b_{i, m}=0$ since $b_{i, m}$ is homogeneous of degree $m$. Iterating this argument we obtain that $b_{i}=r_{i, p}$ hence $\left\{\mathrm{Op}^{\mathrm{w}}\left(b_{i}\right)\right\}_{i \in \mathbb{N}}$ is bounded in $B\left(H^{s}\left(\mathbb{R}^{n}\right), H^{s-m+p}\left(\mathbb{R}^{n}\right)\right)$. But this implies that $A \in B\left(H^{s}(M), H^{s-m+p}(M)\right)$, using the characterization of Sobolev spaces in 2.3.3. Since $p$ is arbitrary we have $A \in \mathcal{W}^{-\infty}(M)$.

5.4. Egorov's theorem. Let us consider an operator $\epsilon(t)=\epsilon_{1}(t)+\epsilon_{0}(t)$, such that:

$$
\epsilon_{i}(t) \in C_{\mathrm{b}}^{\infty}\left(I ; \Psi^{i}(M)\right), i=0,1
$$

$\epsilon_{1}(t)$ is elliptic, symmetric and bounded from below on $H^{\infty}(M)$.

(see Def. 5.9p. By Prop. 5.11 we know that $\epsilon_{1}(t)$ with domain $\operatorname{Dom} \epsilon(t)=H^{1}(M)$ is selfadjoint, hence $\epsilon(t)$ with the same domain is closed, with non empty resolvent set. We denote by $\mathcal{U}_{\epsilon}(t, s)$ the associated propagator defined by:

$$
\left\{\begin{array}{l}
\frac{\partial}{\partial t} \mathcal{U}_{\epsilon}(t, s)=\mathrm{i} \epsilon(t) \mathcal{U}_{\epsilon}(t, s), t, s \in I, \\
\frac{\partial}{\partial s} \mathcal{U}_{\epsilon}(t, s)=-\mathrm{i} \mathcal{U}_{\epsilon}(t, s) \epsilon(s), t, s \in I, \\
\mathcal{U}_{\epsilon}(s, s)=\mathbb{1}, s \in I .
\end{array}\right.
$$

Note that the propagator $\mathcal{U}_{\epsilon_{1}}(t, s)$ exists and is unitary on $L^{2}(M)$, by e.g. $\mathrm{RS}$, Thm. X.70]. Since $\epsilon(t)-\epsilon_{1}(t)$ is uniformly bounded in $B\left(L^{2}(M)\right)$, one easily deduces the existence of $\mathcal{U}_{\epsilon}(t, s)$, which is strongly continuous in $(t, s) \in I^{2}$ with values in $B\left(L^{2}(M)\right)$, uniformly bounded on $I^{2}$ in $B\left(L^{2}(M)\right)$.

Lemma 5.14. Assume (E). Then

(1) $\mathcal{U}_{\epsilon}(t, s) \in B\left(H^{m}(M)\right)$ for $m \in \mathbb{Z} \cup\{ \pm \infty\}, I^{2} \ni(t, s) \mapsto \mathcal{U}_{\epsilon}(t, s)$ is strongly continuous on $H^{m}(M)$,

(2) if $r_{-\infty} \in \mathcal{W}^{-\infty}(M)$ then $\mathcal{U}_{\epsilon}(t, s) r_{-\infty}, r_{-\infty} \mathcal{U}_{\epsilon}(t, s) \in C_{\mathrm{b}}^{\infty}\left(I_{t, s}^{2}, \mathcal{W}^{-\infty}(M)\right)$.

Proof. Note that (2) follows from (1). If clearly suffices to prove (1) for $m$ finite. We set $a=\left(-\Delta_{g}+\mathbb{1}\right)^{\frac{1}{2}}$ and compute

$$
\begin{aligned}
& \partial_{t}\left(\mathcal{U}_{\epsilon}(s, t) a^{m} \mathcal{U}_{\epsilon}(t, s) a^{-m}\right) \\
& =-\mathrm{i} \mathcal{U}_{\epsilon}(s, t)\left[\epsilon(t), a^{m}\right] \mathcal{U}_{\epsilon}(t, s) a^{-m} \\
& =-\mathrm{i} \mathcal{U}_{\epsilon}(s, t) \times\left[\epsilon(t), a^{m}\right] a^{-m} \times a^{m} \mathcal{U}_{\epsilon}(t, s) a^{-m} .
\end{aligned}
$$

We know that $a^{m} \in \Psi^{m}(M)$, hence $\left[\epsilon(t), a^{m}\right] a^{-m} \in C^{\infty}\left(I ; \Psi^{0}(M)\right)$. Moreover $\mathcal{U}_{\epsilon}(t, s)$ is locally bounded in $B\left(L^{2}(M)\right)$ on $I^{2}$. Therefore

$$
\partial_{t}\left\|\mathcal{U}_{\epsilon}(s, t) a^{m} \mathcal{U}_{\epsilon}(t, s) a^{-m} u\right\| \leq C\left\|\mathcal{U}_{\epsilon}(s, t) a^{m} \mathcal{U}_{\epsilon}(t, s) a^{-m} u\right\|, \quad(t, s) \in I^{2}, u \in L^{2}(M) .
$$

For $m<0$, taking $u \in \operatorname{Dom} a^{-m}$ and using Gronwall's inequality yields (1). For $m>0$ we argue similarly, replacing the unbounded operator $a^{m}$ by $a_{\delta}^{m}=a^{m}(1+$ $\mathrm{i} \delta a)^{-m}$ for $\delta>0$. We obtain from Gronwall's inequality that:

$$
\left\|\mathcal{U}_{\epsilon}(s, t) a_{\delta}^{m} \mathcal{U}_{\epsilon}(t, s) a^{-m}\right\| \leq C\left\|a_{\delta}^{m} a^{-m}\right\|,(t, s) \in I^{2} .
$$

We conclude the proof by using that $\left\|a^{m} u\right\|=\sup _{0<\delta}\left\|a_{\delta}^{m} u\right\| . \square$ 
The following theorem is a version of Egorov's theorem.

Theorem 5.15. Let $a \in \Psi^{m}(M)$ and $\epsilon(t)$ satisfying $(E)$. Then

$$
a(t, s):=\mathcal{U}_{\epsilon}(t, s) a \mathcal{U}_{\epsilon}(s, t) \in C_{\mathrm{b}}^{\infty}\left(I^{2}, \Psi^{m}(M)\right) .
$$

Moreover

$$
\sigma_{\mathrm{pr}}(a)(t, s)=\sigma_{\mathrm{pr}}(a) \circ \Phi(s, t),
$$

where $\Phi(t, s): T^{*} M \rightarrow T^{*} M$ is the flow of the time-dependent Hamiltonian $\sigma_{\mathrm{pr}}(\epsilon)(t)$.

Proof. The proof consists of several steps.

Step 1: we write $a=\mathrm{Op}(c)+a_{-\infty}, c \in S_{\mathrm{ph}}^{m}\left(T^{*} M\right), a_{-\infty} \in \mathcal{W}^{-\infty}$. Then

$$
\begin{aligned}
& \mathcal{U}_{\epsilon}(t, s) a \mathcal{U}_{\epsilon}(s, t)=\mathcal{U}_{\epsilon}(t, s) \operatorname{Op}(c) \mathcal{U}_{\epsilon}(s, t)+\mathcal{U}_{\epsilon}(t, s) a_{-\infty} \mathcal{U}_{\epsilon}(s, t) \\
& =\mathcal{U}_{\epsilon}(t, s) \operatorname{Op}(c) \mathcal{U}_{\epsilon}(s, t)+C_{\mathrm{b}}^{\infty}\left(I^{2}, \mathcal{W}^{-\infty}(M)\right),
\end{aligned}
$$

by Lemma 5.14. Therefore we can assume that $a=\mathrm{Op}(c)$.

Step 2: we write $\epsilon(t)=\operatorname{Op}(b)(t)+\epsilon_{-\infty}(t)$ for $b(t) \in C_{\mathrm{b}}^{\infty}\left(I ; S_{\mathrm{ph}}^{1}\left(T^{*} M\right)\right), \epsilon_{-\infty}(t) \epsilon$ $C_{\mathrm{b}}^{\infty}\left(I^{2}, \mathcal{W}^{-\infty}(M)\right)$.

We write:

$$
\mathcal{U}_{\epsilon}(t, s)=: \mathcal{U}_{\mathrm{Op}(b)}(t, s) \mathcal{V}(t, s)
$$

where

$$
\left\{\begin{array}{l}
\partial_{t} \mathcal{V}(t, s)=-\mathrm{i} \mathcal{U}_{\mathrm{Op}(b)}(s, t) \epsilon_{-\infty}(t) \mathcal{U}_{\epsilon}(t, s)=: \tilde{\epsilon}_{-\infty}(t, s), \\
\mathcal{V}(s, s)=\mathbb{1} .
\end{array}\right.
$$

By Lemma 5.14 we know that $\tilde{\epsilon}_{-\infty}(t, s) \in C_{\mathrm{b}}^{\infty}\left(I^{2}, \mathcal{W}^{-\infty}(M)\right)$, hence

$$
\mathcal{V}(t, s)=\mathbb{1}+C_{\mathrm{b}}^{\infty}\left(I^{2}, \mathcal{W}^{-\infty}(M)\right) .
$$

It follows that:

$$
\begin{aligned}
& \mathcal{U}_{\epsilon}(t, s) \operatorname{Op}(c) \mathcal{U}_{\epsilon}(s, t)=\mathcal{U}_{\mathrm{Op}(b)}(t, s) \mathcal{V}(t, s) \operatorname{Op}(c) \mathcal{V}(s, t) \mathcal{U}_{\mathrm{Op}(b)}(s, t) \\
& =\mathcal{U}_{\mathrm{Op}(b)}(t, s) \mathrm{Op}(c) \mathcal{U}_{\mathrm{Op}(b)}(s, t)+C_{\mathrm{b}}^{\infty}\left(I^{2}, \mathcal{W}^{-\infty}(M)\right),
\end{aligned}
$$

again by Lemma 5.14. Therefore it suffices to consider

$$
a_{1}(t, s):=\mathcal{U}_{\mathrm{Op}(b)}(t, s) \mathrm{Op}(c) \mathcal{U}_{\mathrm{Op}(b)}(s, t) .
$$

Step 3: We try to construct $d(t, s) \in C_{\mathrm{b}}^{\infty}\left(I^{2}, S_{\mathrm{ph}}^{m}\left(T^{*} M\right)\right)$ such that

$$
\left\{\begin{array}{l}
\partial_{t} \mathrm{Op}(d)(t, s)=-[\mathrm{Op}(b)(t), \operatorname{iOp}(d)(t, s)], t, s \in I, \\
\mathrm{Op}(d)(s, s)=\mathrm{Op}(c), s \in I,
\end{array}\right.
$$

modulo error terms in $\mathcal{W}^{-\infty}(M)$. As in [Ta, Sec. 0.9], we write

$$
\begin{aligned}
& c \simeq \sum_{i \in \mathbb{N}} c_{m-i}, c_{m-i} \in S_{\mathrm{h}}^{m-i}\left(T^{*} M\right), \\
& b(t) \simeq \sum_{i \in \mathbb{N}} b_{1-i}(t), b_{1}(t)=\sigma_{\mathrm{pr}}(\epsilon)(t), b_{1-i}(t) \in C^{\infty}\left(I ; S_{\mathrm{ph}}^{1-i}\left(T^{*} M\right)\right),
\end{aligned}
$$

and solve 5.4 with the ansatz

$$
d(t, s) \simeq \sum_{i \in \mathbb{N}} d_{m-i}(t, s), d_{m-i} \in C^{\infty}\left(I ; S_{\mathrm{h}}^{m-i}\left(T^{*} M\right)\right)
$$

We obtain the sequence of transport equations:

$$
\begin{aligned}
& (E 0)\left\{\begin{array}{l}
\partial_{t} d_{m}(t, s)+\left\{\sigma_{\mathrm{pr}}(\epsilon(t)), d_{m}(t, s)\right\}=0, \\
d_{m}(s, s)=c_{m}
\end{array}\right. \\
& (E i)\left\{\begin{array}{l}
\partial_{t} d_{m-i}(t, s)+\left\{\sigma_{\mathrm{pr}}(\epsilon(t)), d_{m}(t, s)\right\}=\sum_{-j+m-k+1-l=m-i} P_{j}\left(d_{m-k}, b_{1-l}\right)(t, s), \\
d_{m-i}(s, s)=0,
\end{array}\right.
\end{aligned}
$$


where $\{\cdot, \cdot\}$ is the Poisson bracket and

$$
P_{j}: S_{\mathrm{h}}^{p_{1}}\left(T^{*} M\right) \times S_{\mathrm{h}}^{p_{2}}\left(T^{*} M\right) \rightarrow S_{\mathrm{h}}^{p_{1}+p_{2}-j}\left(T^{*} M\right)
$$

is a bi-differential operator homogeneous of degree $j$ (see [ALNV, Sect. 1.1]).

This sequence of transport equations can be solved inductively in

$$
C_{\mathrm{b}}^{\infty}\left(I ; S_{\mathrm{h}}^{m-i}\left(T^{*} M\right)\right),
$$

using that $\sigma_{\mathrm{pr}}(\epsilon(t))$ is real-valued and elliptic. We have in particular:

$$
d_{m}(t, s)=c_{m} \circ \Phi(s, t) .
$$

Now we choose $d(t, s) \simeq \sum_{i \in \mathbb{N}} d_{m-i}(t, s)$ and obtain:

$$
\left\{\begin{array}{l}
\left.\partial_{t} \mathrm{Op}(d)(t, s)=-[\mathrm{Op}(b)(t), \mathrm{iOp}(d)(t, s))\right]+C_{\mathrm{b}}^{\infty}\left(I^{2}, \mathcal{W}^{-\infty}(M)\right) \\
\mathrm{Op}(d)(s, s)=\mathrm{Op}(c)+C_{\mathrm{b}}^{\infty}\left(I ; \mathcal{W}^{-\infty}(M)\right) .
\end{array}\right.
$$

It follows that

$$
\begin{aligned}
& \partial_{t}\left(\mathcal{U}_{\mathrm{Op}(b)}(s, t) \mathrm{Op}(d)(t, s) \mathcal{U}_{\mathrm{Op}(b)}(t, s)\right) \\
& =\mathcal{U}_{\mathrm{Op}(b)}(s, t)\left(\partial_{t} \mathrm{Op}(d)(t, s)-\mathrm{i}[\mathrm{Op}(b)(t), \mathrm{Op}(d)(t, s)]\right) \mathcal{U}_{\mathrm{Op}(b)}(t, s) \\
& \in C_{\mathrm{b}}^{\infty}\left(I^{2}, \mathcal{W}^{-\infty}(M)\right), \\
& \mathcal{U}_{\mathrm{Op}(b)}(s, s) \mathrm{Op}(d)(s, s) \mathcal{U}_{\mathrm{Op}(b)}(s, s)=\mathrm{Op}(c)+\mathcal{W}^{-\infty}(M) .
\end{aligned}
$$

Hence by integrating from $s$ to $t$ and using again Lemma 5.14 .

$$
\mathrm{Op}(d)(t, s)=a_{1}(t, s)+C_{\mathrm{b}}^{\infty}\left(I^{2}, \mathcal{W}^{-\infty}(M)\right) .
$$

Hence $a_{1}(t, s) \in C^{\infty}\left(I^{2}, \Psi^{m}(M)\right)$ as claimed. By (5.5) we have:

$$
\sigma_{\mathrm{pr}}\left(a_{1}(t, s)\right)=\sigma_{\mathrm{pr}}(a) \circ \Phi(s, t) .
$$

The proof is complete.

5.5. The wave front set. In this subsection we recall the characterization of the wave front set of a distribution $u \in \mathcal{E}^{\prime}(M)$ using pseudodifferential operators on $M$. One says that $A \in \Psi^{m}(M)$ is elliptic at $\left(x_{0}, \xi_{0}\right) \in T^{*} M \backslash\{0\}$ if

$$
\sigma_{\mathrm{pr}}(A)\left(x_{0}, \xi_{0}\right) \neq 0 \text {. }
$$

Proposition 5.16. Let $u \in \mathcal{D}^{\prime}(M)$. Then $\left(x_{0}, \xi_{0}\right) \in T^{*} M \backslash\{0\}$ does not belong to $\mathrm{WF}(u)$ iff there exists $A \in \Psi^{0}(M)$, elliptic at $\left(x_{0}, \xi_{0}\right)$ and $\chi \in C_{0}^{\infty}(M)$ with $\chi\left(x_{0}\right) \neq 0$ such that $A \chi u \in H^{\infty}(M)$, or equivalently $\chi A \chi u \in C_{0}^{\infty}(M)$.

Let us also recall some more notation. If $M_{i}, i=1,2$ are two manifolds one identifies $T^{*}\left(M_{1} \times M_{2}\right)$ and $T^{*} M_{1} \times T^{*} M_{2}$. If $K: C_{0}^{\infty}\left(M_{2}\right) \rightarrow \mathcal{D}^{\prime}\left(M_{1}\right)$ is continuous, denoting again by $K \in \mathcal{D}^{\prime}\left(M_{1} \times M_{2}\right)$ its distributional kernel, one sets:

$$
\mathrm{WF}(K)^{\prime}:=\left\{\left(X_{1}, X_{2}\right) \in\left(T^{*} M_{1} \times T^{*} M_{2}\right) \backslash\{0\}:\left(X_{1}, \bar{X}_{2}\right) \in \mathrm{WF}(K)\right\},
$$

where $\overline{(x, \xi)}=(x,-\xi)$.

Proposition 5.17. Let $\mathcal{U}_{\epsilon}(t, s)$ be as in Thm. 5.15, Then:

$$
\begin{aligned}
& \operatorname{WF}\left(\mathcal{U}_{\epsilon}(t, s) u\right)=\Phi(t, s)(\mathrm{WF}(u)), u \in H^{-\infty}(M), \\
& \operatorname{WF}\left(\mathcal{U}_{\epsilon}(t, s)\right)^{\prime}=\left\{(X, Y) \in T^{*} M \backslash\{0\} \times T^{*} M \backslash\{0\}: X=\Phi(t, s)(Y)\right\}
\end{aligned}
$$

Proof. This follows immediately from Prop. 5.16. Thm. 5.15 and the fact that $\mathcal{U}_{\epsilon}(t, s)$ preserves $H^{\infty}(M)$. 


\section{Parametrices and Propagators}

6.1. Introduction. In this section we consider a class of model Klein-Gordon equations of the form

$$
(\mathrm{KG}) \bar{\partial}_{t}^{2} \phi+r(t, x) \bar{\partial}_{t} \phi+a\left(t, x, \bar{\partial}_{x}\right) \phi=0
$$

on $I_{t} \times \Sigma$, where $I \subset \mathbb{R}$ is an open interval. We will see that the Klein-Gordon equations introduced in Subsect. 3.3 can be reduced to such model equations. We will consider the associated Cauchy evolution operator $\mathcal{U}_{A}(t, s)$, mapping $\rho(s) \phi$ to $\rho(t) \phi$ for $\rho(t) \phi:=\left(\begin{array}{c}\phi(t) \\ \mathrm{i}^{-1} \partial_{t} \phi(t)\end{array}\right)$. It is well-known (see e.g. [Ch]) that $\mathcal{U}_{A}(t, s)$ can expressed microlocally as the sum of two Fourier integral operators, associated with the symplectic flow $\Phi^{ \pm}(t, s)$ generated by $\pm\left(a_{2}(t, x, \xi)\right)^{\frac{1}{2}}$, where $a_{2}(t, x, \xi)$ is the principal symbol of $a\left(t, x, \bar{\partial}_{x}\right)$.

This fact is not sufficient for our purposes, namely the construction of pure Hadamard states for Klein-Gordon fields. We need a more precise decomposition of $\mathcal{U}_{A}(t, s)$ as a sum

$$
\mathcal{U}_{A}(t, s)=\mathcal{U}_{A}^{+}(t, s)+\mathcal{U}_{A}^{-}(t, s)
$$

which we call a microlocal decomposition (see Subsect. 6.5). The essential properties required of $\mathcal{U}_{A}^{ \pm}(t, s)$ is that they are evolutions groups, propagate the wave front set by the flows $\Phi^{ \pm}(t, s)$ and that their ranges are symplectically orthogonal for the natural symplectic form preserved by $\mathcal{U}_{A}(t, s)$.

On a technical level, we avoid the use of the Fourier integral operators machinery and rely instead on propagators $\mathcal{U}_{b}(t, s)$ generated by time-dependent $\Psi$ DOs, which were studied in Subsect. 5.4. As a by-product of the construction of $\mathcal{U}_{A}^{ \pm}(t, s)$, we also obtain a Feynman inverse for the operator $P$ in $(\mathrm{KG})$, canonically associated with the corresponding state, see Subsect. 6.6.

6.2. The model Klein-Gordon equation. In this subsection we give the precise assumptions on our model Klein-Gordon operator (KG), to which the Klein-Gordon operators considered in Subsect. 3.3 can be reduced.

We fix an open interval $I \subset \mathbb{R}$ with $0 \in I$ and a smooth $d$-dimensional manifold $\Sigma$, equipped with a Riemannian metric $k_{0}$, such that $\left(\Sigma, k_{0}\right)$ is of bounded geometry.

We fix the following objects:

(1) a time-dependent Riemannian metric $h_{t}$ on $\Sigma$ such that $h_{t} \in C_{\mathrm{b}}^{\infty}\left(I ; \mathrm{BT}_{2}^{0}\left(\Sigma, k_{0}\right)\right)$ and $h_{t}^{-1} \in C_{\mathrm{b}}^{\infty}\left(I ; \mathrm{BT}_{0}^{2}\left(\Sigma, k_{0}\right)\right)$

(2) a differential operator $a\left(t, x, \bar{\partial}_{x}\right) \in C_{\mathrm{b}}^{\infty}\left(I ; \operatorname{Diff}^{2}\left(\Sigma, k_{0}\right)\right)$ such that

$$
\begin{aligned}
\text { i) } & \sigma_{\mathrm{pr}}(a)(t, x, \xi)=\xi \cdot h_{t}^{-1}(x) \xi, \\
\text { ii) } & a\left(t, x, \bar{\partial}_{x}\right)=a^{*}\left(t, x, \bar{\partial}_{x}\right)
\end{aligned}
$$

where the adjoint is defined using the time-dependent scalar product

$$
(u \mid v)=\int_{\Sigma} \bar{u} v\left|h_{t}\right|^{\frac{1}{2}} d x .
$$

We define then the model Klein-Gordon operator:

$$
P=\bar{\partial}_{t}^{2}+r(t, x) \bar{\partial}_{t}+a\left(t, x, \bar{\partial}_{x}\right)
$$

for $r(t, x):=\left|h_{t}\right|^{-\frac{1}{2}} \partial_{t}\left|h_{t}\right|^{\frac{1}{2}}$. This way $P$ is formally selfadjoint for the scalar product:

$$
(u \mid v)_{M}=\int_{I \times \Sigma} \bar{u} v\left|h_{t}\right|^{\frac{1}{2}} d t d x .
$$


6.3. Solutions to a Riccati equation. Let us abbreviate $a\left(t, x, \bar{\partial}_{x}\right), r(t, x)$ simply by $a, r$. The essential step in the construction of parametrices of the Cauchy problem for the model Klein-Gordon equation introduced in Subsect. 6.2 is to find time-dependent operators $b^{ \pm}(t) \in C^{\infty}\left(I ; \Psi^{1}(\Sigma)\right)$ such that the associated evolution operators $\mathcal{U}_{b^{ \pm}}(t, s)$ solve

$$
\left(\bar{\partial}_{t}^{2}+r \bar{\partial}_{t}+a\right) \mathcal{U}_{b^{ \pm}}(t, s)=0, \text { modulo smoothing errors. }
$$

The above equation is equivalent to the following Riccati equation:

$$
\mathrm{i} \partial_{t} b^{ \pm}-b^{ \pm 2}+a+\mathrm{i} r b^{ \pm}=0,
$$

again modulo smoothing errors. In GW1 6.2 was solved in the special case $\Sigma=$ $\mathbb{R}^{d}, r=0$, using the uniform pseudodifferential calculus on $\mathbb{R}^{d}$, and an equivalent equation (see (6.10) was solved before by Junker in the case of $\Sigma$ compact [Ju1, Ju2 . In this subsection we extend the construction to the case when $\Sigma$ is a manifold of bounded geometry, using the pseudodifferential calculus described in Sect. 5 . allowing also for $r \neq 0$.

Applying Prop. 5.11 to $a$, we can find $c>0$ and $c_{-\infty} \in C_{\mathrm{b}}^{\infty}\left(I ; \mathcal{W}^{-\infty}(\Sigma)\right)$ such that $a(t)+c_{-\infty}(t) \geq c 1$ for $t \in I$. We set $\epsilon(t)=\left(a(t)+c_{-\infty}(t)\right)^{\frac{1}{2}}$, so that $\epsilon^{2}(t)=a(t)+C_{\mathrm{b}}^{\infty}\left(I ; \mathcal{W}^{-\infty}(\Sigma)\right)$. Since $a$ is elliptic, we know from Thm. 5.12 that $\epsilon \in C_{\mathrm{b}}^{\infty}\left(\mathbb{R}, \Psi^{1}(\Sigma)\right)$, with principal symbol $\left(\xi \cdot h_{t}^{-1}(x) \xi\right)^{\frac{1}{2}}$.

Theorem 6.1. There exists $b \in C_{\mathrm{b}}^{\infty}\left(I ; \Psi^{1}(\Sigma)\right)$, unique modulo $C_{\mathrm{b}}^{\infty}\left(I ; \mathcal{W}^{-\infty}(\Sigma)\right)$ such that

$$
\begin{aligned}
& \text { i) } b=\epsilon+C_{\mathrm{b}}^{\infty}\left(I ; \Psi^{0}(\Sigma)\right), \\
& \text { ii) }\left(b+b^{*}\right)^{-1}=(2 \epsilon)^{-\frac{1}{2}}\left(\mathbb{1}+r_{-1}\right)(2 \epsilon)^{-\frac{1}{2}}, r_{-1} \in C_{\mathrm{b}}^{\infty}\left(I ; \Psi^{-1}(\Sigma)\right), \\
& \text { iii) }\left(b+b^{*}\right)^{-1} \geq c \epsilon^{-1}, \text { for some } c \in C_{\mathrm{b}}^{\infty}(I ; \mathbb{R}), c>0 \\
&\text { iv }) \quad \mathrm{i} \partial_{t} b^{ \pm}-b^{ \pm 2}+a+\mathrm{i} r b^{ \pm}=r_{-\infty}^{ \pm} \in C_{\mathrm{b}}^{\infty}\left(I ; \mathcal{W}^{-\infty}(\Sigma)\right), \\
& \text { for } b^{+}:=b, b^{-}:=-b^{*} .
\end{aligned}
$$

Proof. We follow the proof in GW1, Appendix A3]. We can first replace in 6.2 $a$ by $\epsilon^{2}$, modulo an error term in $C_{\mathrm{b}}^{\infty}\left(I ; \mathcal{W}^{-\infty}(\Sigma)\right)$. Discarding error terms in $C_{\mathrm{b}}^{\infty}\left(I ; \mathcal{W}^{-\infty}(\Sigma)\right)$, we can assume that $\epsilon=\mathrm{Op}(c), c \in C_{\mathrm{b}}^{\infty}\left(I ; S_{\mathrm{ph}}^{1}\left(T^{*} \Sigma\right)\right)$, with $c_{\mathrm{pr}}(t, x, \xi)=\left(\xi \cdot h_{t}^{-1}(x) \xi\right)^{\frac{1}{2}}$. We look for $b$ of the form $b=\mathrm{Op}(c)+\mathrm{Op}(d)$ for $d \in C_{\mathrm{b}}^{\infty}\left(I ; S_{\mathrm{ph}}^{0}\left(T^{*} \Sigma\right)\right)$. Since $\mathrm{Op}(c)$ is elliptic, it admits parametrices, see Prop. 5.10. We fix a symbol $\hat{c} \in C_{\mathrm{b}}^{\infty}\left(I ; S_{\mathrm{ph}}^{-1}\left(T^{*} \Sigma\right)\right)$ such that $\mathrm{Op}(\hat{c})$ is a parametrix of $\mathrm{Op}(c)$.

The equation 6.2 becomes, modulo error terms in $C_{\mathrm{b}}^{\infty}\left(I ; \mathcal{W}^{-\infty}(\Sigma)\right)$ :

$$
\mathrm{Op}(d)=\frac{\mathrm{i}}{2}\left(\mathrm{Op}(\hat{c}) \mathrm{Op}\left(\partial_{t} c\right)+\mathrm{Op}(\hat{c}) r \mathrm{Op}(c)\right)+F(\mathrm{Op}(d))
$$

$$
F(\mathrm{Op}(d))=\frac{1}{2} \mathrm{Op}(\hat{c})\left(\mathrm{iOp}\left(\partial_{t} d\right)+[\mathrm{Op}(c), \mathrm{Op}(d)]+\mathrm{i} r \mathrm{Op}(d)-\mathrm{Op}(d)^{2}\right) .
$$

From symbolic calculus, we obtain that:

$$
F(\mathrm{Op}(d))=\operatorname{Op}(\tilde{F}(d))+C_{\mathrm{b}}^{\infty}\left(I ; \mathcal{W}^{-\infty}(\Sigma)\right)
$$

for

$$
\tilde{F}(d)=\frac{1}{2} \hat{c} \sharp\left(\mathrm{i} \partial_{t} d+c \sharp d-d \sharp c+\mathrm{i} r \sharp d-d \sharp d\right),
$$

where the operation $\sharp$ (the Moyal product) is recalled in 5.3.4. The equation 6.3 becomes:

$$
d=a_{0}+\tilde{F}(d),
$$


for

$$
a_{0}=\frac{\mathrm{i}}{2}\left(\hat{c} \sharp \partial_{t} c+\hat{c} \sharp r \sharp c\right) \in C_{\mathrm{b}}^{\infty}\left(I ; S_{\mathrm{ph}}^{0}\left(T^{*} \Sigma\right)\right) .
$$

The map $\tilde{F}$ has the following property:

$$
\begin{aligned}
& d_{1}, d_{2} \in C_{\mathrm{b}}^{\infty}\left(I ; S_{\mathrm{ph}}^{0}\left(T^{*} \Sigma\right)\right), d_{1}-d_{2} \in C_{\mathrm{b}}^{\infty}\left(I ; S_{\mathrm{ph}}^{-j}\left(T^{*} \Sigma\right)\right) \\
& \Rightarrow \tilde{F}\left(d_{1}\right)-\tilde{F}\left(d_{2}\right) \in C_{\mathrm{b}}^{\infty}\left(I ; S_{\mathrm{ph}}^{-j-1}\left(T^{*} \Sigma\right)\right) .
\end{aligned}
$$

This allows to solve symbolically (6.4) by setting

$$
d_{-1}=0, d_{n}:=a_{0}+\tilde{F}\left(d_{n-1}\right),
$$

and

$$
d \simeq \sum_{n \in \mathbb{N}} d_{n}-d_{n-1}
$$

which is an asymptotic series since by 6.5 we see that $d_{n}-d_{n-1} \in C_{\mathrm{b}}^{\infty}\left(I ; S_{\mathrm{ph}}^{-n}\left(T^{*} \Sigma\right)\right)$. It follows that $\mathrm{Op}(c+d)$ solves 6.2 modulo $C_{\mathrm{b}}^{\infty}\left(I ; \mathcal{W}^{-\infty}(\Sigma)\right)$.

We observe then that if $b \in C_{\mathrm{b}}^{\infty}\left(I ; \Psi^{\infty}(\Sigma)\right)$ we have:

$$
\left(\partial_{t} b\right)^{*}=\partial_{t}\left(b^{*}\right)+r b^{*}-b^{*} r
$$

(recall that the adjoint is computed w.r.t the time-dependent scalar product (6.1)). This implies that $-\mathrm{Op}(d)^{*}$ is also a solution of 6.2 modulo $C_{\mathrm{b}}^{\infty}\left(I ; \mathcal{W}^{-\infty}(\Sigma)\right)$.

To complete the construction of $b^{ \pm}$, we consider

$$
s=\mathrm{Op}(c+d)+\mathrm{Op}(c+d)^{*},
$$

which is selfadjoint, with principal symbol equal to $2\left(\xi \cdot h_{t}^{-1}(x) \xi\right)^{\frac{1}{2}}$. By Prop. 5.11 . there exists $r_{-\infty} \in C_{\mathrm{b}}^{\infty}\left(I ; \mathcal{W}^{-\infty}(\Sigma)\right)$ such that

$$
s+r_{-\infty} \sim \epsilon,
$$

where we recall that the notation $\sim$ is defined in 1.8 . We set now:

$$
b:=\mathrm{Op}(c+d)+\frac{1}{2} r_{-\infty} .
$$

Properties i) and $i v$ ) follow from the same properties of $\mathrm{Op}(c+d)$. Property iii) follows from (6.6) and the Kato-Heinz theorem. To prove property ii) we write

$$
b+b^{*}=(2 \epsilon)^{\frac{1}{2}}\left(\mathbb{1}+\tilde{r}_{-1}\right)(2 \epsilon)^{\frac{1}{2}},
$$

where $\tilde{r}_{-1} \in C_{\mathrm{b}}^{\infty}\left(I ; \Psi^{-1}(\Sigma)\right)$, by Thm. 5.12 . Since $\left(\mathbb{1}+\tilde{r}_{-1}\right)$ is boundedly invertible, we have again by Thm. 5.12

$$
\left(\mathbb{1}+\tilde{r}_{-1}\right)^{-1}=\mathbb{1}+r_{-1}, r_{-1} \in C_{\mathrm{b}}^{\infty}\left(I ; \Psi^{-1}(\Sigma)\right),
$$

which implies ii). The proof is complete.

Note that by iv) one has (by subtracting the two identities)

$$
r=\mathrm{i}^{-1}\left(b^{+}+b^{-}\right)-\left(b^{+}-b^{-}\right)^{-1} \partial_{t}\left(b^{+}-b^{-}\right)
$$

modulo smoothing errors. Thus, the pair $b^{ \pm}$contains full information about $r$, and thus about $a$ (using $i v$ ) again).

6.4. Approximate diagonalization. In this subsection we perform a diagonalization modulo smoothing errors of the Cauchy evolution operator $\mathcal{U}_{A}(t, s)$, see 6.4 .1

We extend the notation in Sect. 5 to matrix-valued symbols, operators, etc., by introducing the sets $C_{\mathrm{b}}^{\infty}\left(I ; \Psi^{m}\left(\Sigma, \mathbb{C}_{p}^{n}\right)\right), n, p \in \mathbb{N}$ etc. We will frequently omit the extra symbol $\mathbb{C}_{p}^{n}$ when the nature of the objects is clear from the context. We also extend to this situation the notation $\mathcal{U}_{\epsilon}(t, s)$ when $\epsilon \in C_{\mathrm{b}}^{\infty}\left(I ; \Psi^{m}\left(\Sigma, \mathbb{C}_{n}^{n}\right)\right)$. 
6.4.1. KG equation as a first order system. As usual we write

$$
\left(\partial_{t}^{2}+r(t) \partial_{t}+a(t)\right) \phi(t)=0
$$

as a first order system:

$$
\mathrm{i}^{-1} \partial_{t} \psi(t)=A(t) \psi(t), \quad \text { where } A(t)=\left(\begin{array}{cc}
0 & \mathbb{1} \\
a(t) & \mathrm{i} r(t)
\end{array}\right),
$$

by setting

$$
\psi(t)=\left(\begin{array}{c}
\phi(t) \\
\mathrm{i}^{-1} \partial_{t} \phi(t)
\end{array}\right)=: \rho(t) \phi
$$

We equip $L^{2}\left(\Sigma ; \mathbb{C}^{2}\right)$ with the time-dependent scalar product obtained from (6.1), by setting:

$$
(f \mid g):=\int_{\Sigma}\left(\bar{f}_{1} g_{1}+\bar{f}_{0} g_{0}\right)\left|h_{t}\right|^{\frac{1}{2}} d x .
$$

We will use it to define adjoints of linear operators and to identify sesquilinear forms on $L^{2}\left(\Sigma ; \mathbb{C}^{2}\right)$ with linear operators. Note that if $\phi_{i}$ are $C^{\infty}$ solutions with $\phi_{i} \uparrow_{\Sigma}$ compactly supported then

$$
\mathrm{i} \bar{\phi}_{1} \cdot \sigma \phi_{2}=\left(\rho(t) \phi_{1} \mid q \rho(t) \phi_{2}\right)
$$

for:

$$
q:=\left(\begin{array}{ll}
0 & 1 \\
1 & 0
\end{array}\right)
$$

is independent on $t$. The evolution operator $\mathcal{U}_{A}(t, s)$ is symplectic:

$$
q=\mathcal{U}_{A}^{*}(s, t) q \mathcal{U}_{A}(s, t), s, t \in I .
$$

6.4.2. First reduction. The Riccati equation

$$
\mathrm{i} \partial_{t} b^{ \pm}-b^{ \pm 2}+a+\mathrm{i} r b^{ \pm}=r_{-\infty}^{ \pm}
$$

implies that:

$$
\left(\bar{\partial}_{t}+\mathrm{i} b^{ \pm}+r\right) \circ\left(\bar{\partial}_{t}-\mathrm{i} b^{ \pm}\right)=\bar{\partial}_{t}^{2}+r \bar{\partial}_{t}+a-r_{-\infty}^{ \pm}
$$

which is a factorization of the Klein-Gordon operator $P$ modulo smoothing errors. One can also deduce from 6.10 a time-dependent diagonalization of the evolution operator for $P$, which we now define. We set

$$
\tilde{\psi}(t):=\left(\begin{array}{l}
\bar{\partial}_{t}-\mathrm{i} b^{-}(t) \\
\bar{\partial}_{t}-\mathrm{i} b^{+}(t)
\end{array}\right) \phi(t)
$$

and obtain $\tilde{\psi}(t)=S^{-1}(t) \psi(t)$ with

$$
S^{-1}(t)=\mathrm{i}\left(\begin{array}{cc}
-b^{-}(t) & 1 \\
-b^{+}(t) & 1
\end{array}\right), \quad S(t)=\mathrm{i}^{-1}\left(\begin{array}{cc}
1 & -1 \\
b^{+}(t) & -b^{-}(t)
\end{array}\right)\left(b^{+}(t)-b^{-}(t)\right)^{-1},
$$

which makes sense thanks to $b^{+}(t)-b^{-}(t)$ being invertible by Thm. 6.1. We obtain from 6.10 that

$$
\begin{aligned}
& \left(\begin{array}{cc}
\bar{\partial}_{t}+\mathrm{i} b^{-}+r & 0 \\
0 & \bar{\partial}_{t}+\mathrm{i} b^{+}+r
\end{array}\right) \tilde{\psi}(t)=\left(\begin{array}{c}
\bar{\partial}_{t}^{2}+a+r \bar{\partial}_{t}-r_{-\infty}^{-} \\
\bar{\partial}_{t}^{2}+a+r \bar{\partial}_{t}-r_{-\infty}^{+}
\end{array}\right) \phi(t) \\
& =\left(\begin{array}{c}
P \phi \\
P \phi
\end{array}\right)-\left(\begin{array}{cc}
r_{-\infty}^{-} & 0 \\
r_{-\infty}^{+} & 0
\end{array}\right) S(t) \tilde{\psi}(t),
\end{aligned}
$$

Let

$$
B(t)=\tilde{B}(t)+S_{-\infty}(t)
$$


for

$$
\tilde{B}(t)=\left(\begin{array}{cc}
-b^{-}+\mathrm{i} r & 0 \\
0 & -b^{+}+\mathrm{i} r
\end{array}\right), S_{-\infty}(t)=\left(\begin{array}{cc}
r_{-\infty}^{-} & -r_{-\infty}^{-} \\
r_{-\infty}^{+} & -r_{-\infty}^{+}
\end{array}\right)\left(b^{+}-b^{-}\right)^{-1} .
$$

Then since $P \phi=0$ we deduce from 6.12 that:

$$
\left(\bar{\partial}_{t}-\mathrm{i} B(t)\right) \tilde{\psi}(t)=0,
$$

hence:

$$
\mathcal{U}_{A}(t, s)=S(t) \mathcal{U}_{B}(t, s) S(s)^{-1} .
$$

We have thus a formula that relates $\mathcal{U}_{A}(t, s)$ and the evolution generated by a timedependent operator $B(t)$ that is diagonal up to $\mathcal{W}^{-\infty}(\Sigma)$ remainders and whose on-diagonal terms have principal symbols $\pm\left(\xi \cdot h_{t}^{-1}(x) \xi\right)^{\frac{1}{2}}$.

Let us now discuss the symplectic properties of $\mathcal{U}_{B}(t, s)$. Since

$$
S(s)^{*} q S(s)=\left(b^{+}-b^{-}\right)^{-1}(s)\left(\begin{array}{cc}
1 & 0 \\
0 & -1
\end{array}\right)=: q_{B}(s)
$$

we obtain from 6.13, 6.8 that:

$$
q_{B}(t)=\mathcal{U}_{B}^{*}(s, t) q_{B}(s) \mathcal{U}_{B}(s, t), s, t \in I .
$$

6.4.3. Second reduction. To get rid of the $\left(b^{+}-b^{-}\right)^{-1}(s)$ factor in $q_{B}(s)$ we set

$$
\mathcal{U}_{C}(t, s):=\left(b^{+}-b^{-}\right)^{-\frac{1}{2}}(t) \mathcal{U}_{B}(t, s)\left(b^{+}-b^{-}\right)^{\frac{1}{2}}(s) .
$$

It follows that:

$$
\mathcal{U}_{A}(t, s)=T(t) \mathcal{U}_{C}(t, s) T(s)^{-1},
$$

for:

$$
\begin{aligned}
& T(t):=S(t)\left(b^{+}-b^{-}\right)^{\frac{1}{2}}(t)=\mathrm{i}^{-1}\left(\begin{array}{cc}
1 & -1 \\
b^{+} & -b^{-}
\end{array}\right)\left(b^{+}-b^{-}\right)^{-\frac{1}{2}}, \\
& T^{-1}(t)=\mathrm{i}\left(b^{+}-b^{-}\right)^{-\frac{1}{2}}\left(\begin{array}{cc}
-b^{-} & 1 \\
-b^{+} & 1
\end{array}\right) .
\end{aligned}
$$

Note that:

$$
T^{*}(t) q T(t)=\left(\begin{array}{cc}
1 & 0 \\
0 & -1
\end{array}\right)=: \hat{q},
$$

so that $\mathcal{U}_{C}(t, s)$ is symplectic for $\hat{q}$ :

$$
\mathcal{U}_{C}(t, s)^{*} \hat{q} \mathcal{U}_{C}(t, s)=\hat{q} .
$$

The generator of $\mathcal{U}_{C}(t, s)$ is:

$$
C(t)=\tilde{C}(t)+R_{-\infty}(t)
$$

for

$$
\begin{aligned}
\tilde{C}(t) & :=\left(b^{+}-b^{-}\right)^{-\frac{1}{2}} \tilde{B}(t)\left(b^{+}-b^{-}\right)^{\frac{1}{2}}-\mathrm{i} \partial_{t}\left(b^{+}-b^{-}\right)^{-\frac{1}{2}}\left(b^{+}-b^{-}\right)^{\frac{1}{2}} \\
& =\left(\begin{array}{cc}
-b^{-}+r_{0}^{-} & 0 \\
0 & -b^{+}+r_{0}^{+}
\end{array}\right)
\end{aligned}
$$

where

$$
r_{0}^{ \pm}=\mathrm{i} r+\left[\left(b^{+}-b^{-}\right)^{-\frac{1}{2}}, b^{ \pm}\right]-\mathrm{i} \partial_{t}\left(b^{+}-b^{-}\right)^{-\frac{1}{2}}\left(b^{+}-b^{-}\right)^{\frac{1}{2}} \in C_{\mathrm{b}}^{\infty}\left(I ; \Psi^{0}(\Sigma)\right) .
$$

and

$$
R_{-\infty}=-\left(b^{+}-b^{-}\right)^{-\frac{1}{2}} S_{-\infty}\left(b^{+}-b^{-}\right)^{\frac{1}{2}} \in C_{\mathrm{b}}^{\infty}\left(I ; \mathcal{W}^{-\infty}(\Sigma)\right)
$$


Remark 6.2. Let us explain another motivation for the introduction of the maps $T(t)$. There are two natural topologies on the space of Cauchy data for (6.7). The first is the energy space topology given by the topology of $H^{1}(\Sigma) \oplus L^{2}(\Sigma)$, ubiquitous in the PDE literature. The second is the charge space topology, given by the topology of $H^{\frac{1}{2}}(\Sigma) \oplus H^{-\frac{1}{2}}(\Sigma)$, related to the quantization of the Klein-Gordon equation. It is easy to see that $S(t)$ is an isomorphism from $L^{2}(\Sigma) \oplus L^{2}(\Sigma)$ to $H^{1}(\Sigma) \oplus L^{2}(\Sigma)$, while $T(t)$ is an isomorphism from $L^{2}(\Sigma) \oplus L^{2}(\Sigma)$ to $H^{\frac{1}{2}}(\Sigma) \oplus H^{-\frac{1}{2}}(\Sigma)$.

6.4.4. Interaction picture. From 6.18 we know that the generator of $\mathcal{U}_{C}(t, s)$ is diagonal, modulo a smoothing error term. It follows from standard arguments that $\mathcal{U}_{C}(t, s)$ is also diagonal, modulo smoothing errors. We review this argument, known as the 'interaction picture' in the physics literature.

Let $H(t)=H_{0}(t)+V(t)$ be a time-dependent Hamiltonian, $\mathcal{U}(\cdot, \cdot)$ and $\mathcal{U}_{0}(\cdot, \cdot)$ the associated propagators. We fix $t_{1} \in \mathbb{R}$ and set:

$$
\mathcal{U}(t, s)=: \mathcal{U}_{0}\left(t, t_{1}\right) \mathcal{V}_{t_{1}}(t, s) \mathcal{U}_{0}\left(t_{1}, s\right)
$$

(Typically $H_{0}$ does not depend on time and one sets $t_{1}=0$ ). It follows that $\mathcal{V}_{t_{1}}(\cdot, \cdot)$ is an evolution group and solves

$$
\left\{\begin{array}{l}
\partial_{t} \mathcal{V}_{t_{1}}(t, s)=\mathrm{i} \tilde{V}_{t_{1}}(t) \mathcal{V}_{t_{1}}(t, s) \text { for } \tilde{V}_{t_{1}}(t)=\mathcal{U}_{0}\left(t_{1}, t\right) V(t) \mathcal{U}_{0}\left(t, t_{1}\right) \\
\mathcal{V}_{t_{1}}(s, s)=\mathbb{1}
\end{array}\right.
$$

Note the following covariance property:

$$
\mathcal{V}_{t_{2}}(t, s)=\mathcal{U}_{0}\left(t_{1}, t_{2}\right) \mathcal{V}_{t_{1}}(t, s) \mathcal{U}_{0}\left(t_{2}, t_{1}\right), t_{1}, t_{2} \in \mathbb{R}
$$

6.4.5. Parametrix for the Cauchy problem. We apply the above procedure to $C=$ $\tilde{C}+R_{-\infty}$, fix some $t_{1} \in I$ and set:

$$
\mathcal{U}_{C}(t, s)=: \mathcal{U}_{\tilde{C}}\left(t, t_{1}\right) \mathcal{V}_{t_{1}}(t, s) \mathcal{U}_{\tilde{C}}\left(t_{1}, s\right)
$$

where $\mathcal{V}_{t_{1}}(t, s)$ is the evolution generated by $R_{t_{1},-\infty}(t)=\mathcal{U}_{\tilde{C}}\left(t_{1}, t\right) R_{-\infty}(t) \mathcal{U}_{\tilde{C}}\left(t, t_{1}\right)$, i.e.

$$
\left\{\begin{array}{l}
\partial_{t} \mathcal{V}_{t_{1}}(t, s)=\mathrm{i} R_{t_{1},-\infty}(t) \mathcal{V}_{t_{1}}(t, s) \\
\mathcal{V}_{t_{1}}(s, s)=\mathbb{1}
\end{array}\right.
$$

Note that $\tilde{C}(t)$ is diagonal, with entries satisfying condition (E) in Subsect. 5.4 . Therefore by Lemma 5.14 we know that $R_{t_{1},-\infty}(t) \in C_{\mathrm{b}}^{\infty}\left(I ; \mathcal{W}^{-\infty}(\Sigma)\right)$. For any $s \in \mathbb{R}$, the equation (6.21) can be solved in $C_{\mathrm{b}}^{\infty}\left(I^{2} ; B\left(H^{s}(\Sigma)\right)\right)$ by a convergent series. This implies easily that:

$$
\begin{aligned}
& \mathcal{V}_{t_{1}}(t, s)=\mathbb{1}+C_{\mathrm{b}}^{\infty}\left(I^{2}, \Psi^{-\infty}(\Sigma)\right), \\
& \mathcal{U}_{C}(t, s)=\mathcal{U}_{\tilde{C}}(t, s)+C_{\mathrm{b}}^{\infty}\left(I^{2}, \Psi^{-\infty}(\Sigma)\right) .
\end{aligned}
$$

We summarize this discussion with the following theorem.

Theorem 6.3. Let

$$
\mathcal{U}_{\tilde{A}}(t, s):=T(t) \mathcal{U}_{\tilde{C}}(t, s) T(s)^{-1}
$$

Then $\left\{\mathcal{U}_{\tilde{A}}(t, s)\right\}_{(t, s) \in I^{2}}$ is an evolution group and:

$$
\mathcal{U}_{A}(t, s)=\mathcal{U}_{\tilde{A}}(t, s)+C_{\mathrm{b}}^{\infty}\left(I^{2}, \Psi^{-\infty}(\Sigma)\right) .
$$

It follows that the group $\left\{\mathcal{U}_{\tilde{A}}(t, s)\right\}_{(t, s) \in I^{2}}$ is a parametrix for the Cauchy problem.

Note that since $\tilde{C}(t)$ is diagonal, we have:

$$
\mathcal{U}_{\tilde{C}}(t, s)=\left(\begin{array}{cc}
\mathcal{U}_{-b^{-}+r_{0}^{-}}(t, s) & 0 \\
0 & \mathcal{U}_{-b^{+}+r_{0}^{+}}(t, s)
\end{array}\right) .
$$


6.5. Decomposition of the Cauchy evolution. Basing on the constructions in Subsect. 6.4 it is easy to construct a microlocal decomposition of the evolution $\mathcal{U}_{A}(t, s)$. In fact let

$$
\pi^{+}=\left(\begin{array}{ll}
\mathbb{1} & 0 \\
0 & 0
\end{array}\right), \quad \pi^{-}=\left(\begin{array}{ll}
0 & 0 \\
0 & \mathbb{1}
\end{array}\right)
$$

We fix a reference time $t_{0} \in I$ for example $t_{0}=0$ and set:

(6.26) $c^{ \pm}(0):=T(0) \pi^{ \pm} T^{-1}(0)=\left(\begin{array}{cc}\mp\left(b^{+}-b^{-}\right)^{-1} b^{\mp} & \pm\left(b^{+}-b^{-}\right)^{-1} \\ \mp b^{+}\left(b^{+}-b^{-}\right)^{-1} b^{-} & \pm b^{ \pm}\left(b^{+}-b^{-}\right)^{-1}\end{array}\right)(0)$.

We have:

$$
c^{ \pm}(0)^{2}=c^{ \pm}(0), c^{+}(0)+c^{-}(0)=\mathbb{1}, c^{ \pm}(0) \in C_{\mathrm{b}}^{\infty}\left(I ; \Psi^{\infty}(\Sigma)\right) .
$$

It follows that $c^{+}(0), c^{-}(0)$ is a pair of complementary projections. Moreover from 6.16 , we obtain that:

$$
c^{\mp *}(0) q c^{ \pm}(0)=0
$$

i.e. the ranges of the projections $c^{ \pm}(0)$ are $q$-orthogonal. We set:

$$
\mathcal{U}_{A}^{ \pm}(t, s):=\mathcal{U}_{A}(t, 0) c^{ \pm}(0) \mathcal{U}_{A}(0, s) .
$$

Definition 6.4. A pair $\left\{\mathcal{U}_{A}^{ \pm}(t, s)\right\}_{(t, s) \in I^{2}}$ as in 6.28 will be called a microlocal decomposition of the evolution group $\left\{\mathcal{U}_{A}(t, s)\right\}_{(t, s) \in I^{2}}$.

Theorem 6.5. The following properties are true:

i) $\mathcal{U}_{A}^{ \pm}(t, s) \mathcal{U}_{A}^{ \pm}\left(s, t^{\prime}\right)=\mathcal{U}_{A}^{ \pm}\left(t, t^{\prime}\right)$,

ii) $\mathcal{U}_{A}^{+}(t, s)+\mathcal{U}_{A}^{-}(t, s)=\mathcal{U}_{A}(t, s)$,

iii) $\mathcal{U}_{A}^{ \pm}(t, s)^{*} q \mathcal{U}_{A}^{\mp}(t, s)=0$,

iv) $\left(\bar{\partial}_{t}-\mathrm{i} A(t)\right) \mathcal{U}_{A}^{ \pm}(t, s)=\mathcal{U}_{A}^{ \pm}(t, s)\left(\bar{\partial}_{s}-\mathrm{i} A(s)\right)=0$,

v) $\mathrm{WF}\left(\mathcal{U}_{A}^{ \pm}(t, s)\right)^{\prime}=\left\{\left(X, X^{\prime}\right) \in T^{*} \Sigma \times T^{*} \Sigma: X=\Phi^{ \pm}(t, s)\left(X^{\prime}\right)\right\}$,

where $\Phi^{ \pm}(t, s): T^{*} \Sigma \rightarrow T^{*} \Sigma$ is the symplectic flow generated by the time-dependent Hamiltonian $\pm\left(\xi \cdot h_{t}^{-1}(x) \xi\right)^{\frac{1}{2}}$.

Proof. $i$ ) and $i i)$ follow from the fact that $c^{ \pm}(0)$ are complementary projections. iii) follows from (6.8) and (6.27). $i v)$ is immediate. From (6.24) and Prop. 5.17 we obtain that $\mathcal{U}_{C}(t, 0) \pi^{ \pm} \mathcal{U}_{C}(0, s)$ has the wave front set stated in $\left.v\right)$. The result follows then from the fact that $\mathcal{U}_{A}^{ \pm}(t, s)=T(t) \mathcal{U}_{C}(t, 0) \pi^{ \pm} \mathcal{U}_{C}(0, s) T^{-1}(s)$.

We now gather a couple of formulae that relate various objects at different times. The proof is a routine computation that uses the first three statements in Thm. 6.5 .

Proposition 6.6. Let

$$
c^{ \pm}(t):=\mathcal{U}_{A}^{ \pm}(t, t)=\mathcal{U}_{A}(t, 0) c^{ \pm}(0) \mathcal{U}_{A}(0, t) .
$$

Then:

$$
\begin{aligned}
& c^{ \pm}(t)^{2}=c^{ \pm}(t), c^{+}(t)+c^{-}(t)=\mathbb{1}, \\
& c^{ \pm}(t)=\mathcal{U}_{A}(t, s) c^{ \pm}(s) \mathcal{U}_{A}(s, t), \\
& c^{\mp}(t) q c^{ \pm}(t)^{*}=0, c^{ \pm}(t) \mathcal{U}_{A}(t, s) c^{\mp}(s)=0, \\
& \mathcal{U}_{A}^{ \pm}(t, s)=c^{ \pm}(t) \mathcal{U}_{A}(t, s) c^{ \pm}(s)=c^{ \pm}(t) \mathcal{U}_{A}(t, s)=\mathcal{U}_{A}(t, s) c^{ \pm}(s) .
\end{aligned}
$$


6.6. The Feynman inverse associated to a microlocal decomposition. In this subsection we work in the setup of Subsect. 6.2

6.6.1. Distinguished parametrices for the Klein-Gordon operator. In our terminology, a continuous map $G: C_{0}^{\infty}(M) \rightarrow C^{\infty}(M)$ is a (two-sided) parametrix of the Klein-Gordon operator $P$ if $P G-\mathbb{1}$ and $\mathbb{1}-G P$ have smooth kernels. In what follows we recall the classification of parametrices of $P$ due to Duistermaat and Hörmander in $[\mathrm{DH}]$.

For $x \in M$ we denote by $V_{x \pm} \subset T_{x} M$ the future/past solid lightcones and by $V_{x \pm}^{*} \subset T_{x}^{*} M$ the dual cones $V_{x \pm}^{*}=\left\{\xi \in T_{x}^{*} M: \xi \cdot v>0, \forall v \in V_{x \pm}, v \neq 0\right\}$. We write

$$
\xi \triangleright 0 \text { (resp. } \xi \triangleleft 0) \text { if } \xi \in V_{x+}^{*} \text { (resp. } V_{x-}^{*} \text { ). }
$$

For $X=(x, \xi) \in T^{*} M \backslash\{0\}$ denote $p(X)=\xi \cdot g^{-1}(x) \xi$ the principal symbol of $P$ and $\mathcal{N}=p^{-1}(0) \cap T^{*} M \backslash\{0\}$ the characteristic manifold of $P$. If $H_{p}$ is the Hamiltonian vector field of $p$, integral curves of $H_{p}$ in $\mathcal{N}$ are called bicharacteristics. $\mathcal{N}$ splits into the upper/lower energy shells

$$
\mathcal{N}=\mathcal{N}^{+} \cup \mathcal{N}^{-}, \quad \mathcal{N}^{ \pm}=\mathcal{N} \cap\{ \pm \xi \triangleright 0\} .
$$

For $X_{1}, X_{2} \in \mathcal{N}$ we write $X_{1} \sim X_{2}$ if $X_{1}, X_{2}$ lie on the same bicharacteristic. For $X_{1} \sim X_{2}$, we write $X_{1} \succ X_{2}$ (resp. $X_{1} \prec X_{2}$ ) if $X_{1}$ comes strictly after (before) $X_{2}$ w.r.t. the natural parameter on the bicharacteristic through $X_{1}$ and $X_{2}$. Finally one sets

$$
\mathcal{C}=\left\{\left(X_{1}, X_{2}\right) \in \mathcal{N} \times \mathcal{N}: X_{1} \sim X_{2}\right\}, \quad \Delta=\left\{(X, X): X \in T^{*} M \backslash\{0\}\right\},
$$

and

$$
\begin{aligned}
& \mathcal{C}_{\text {ret } / \text { adv }}=\left\{\left(X_{1}, X_{2}\right) \in \mathcal{C}: x_{1} \in J^{ \pm}\left(x_{2}\right)\right\}, \\
& \mathcal{C}_{\mathrm{F}}=\left\{\left(X_{1}, X_{2}\right) \in \mathcal{C}: X_{1} \prec X_{2}\right\}, \\
& \mathcal{C}_{\overline{\mathrm{F}}}=\left\{\left(X_{1}, X_{2}\right) \in \mathcal{C}: X_{1} \succ X_{2}\right\} .
\end{aligned}
$$

The main results of $[\mathrm{DH}]$ relevant to us is the following theorem.

Theorem 6.7. $\mathrm{DH}$, Thm. 6.5.3] For $\sharp=$ ret, adv, F, $\overline{\mathrm{F}}$ there exists a parametrix $G_{\sharp}$ of $P$ such that

$$
\mathrm{WF}\left(G_{\sharp}\right)^{\prime}=\Delta \cup \mathcal{C}_{\sharp} .
$$

Any other parametrix $G$ with $\mathrm{WF}(G)^{\prime} \subset \Delta \cup \mathcal{C}_{\sharp}$ equals $G_{\sharp}$ modulo a smooth kernel.

A parametrix satisfying 6.30 for $\sharp=$ ret/adv resp. $\sharp=F / \bar{F}$ will be called a retarded/advanced resp. Feynman/anti-Feynman parametrix (or inverse if $P G_{\sharp}=\mathbb{1}$ and $G_{\sharp} P=\mathbb{1}$ hold exactly).

6.6.2. The Feynman inverse associated to a microlocal decomposition. We now show how to associate to the decomposition of the Cauchy evolution constructed in Subsect. 6.5 a Feynman inverse for the Klein-Gordon operator $P$.

In the next theorem, we will use the 'time kernel' notation: namely if $A$ : $C_{0}^{\infty}\left(M ; \mathbb{C}^{p}\right) \rightarrow C^{\infty}\left(M ; \mathbb{C}^{q}\right)$ we denote by $A(t, s): C_{0}^{\infty}\left(\Sigma ; \mathbb{C}^{p}\right) \rightarrow C^{\infty}\left(\Sigma ; \mathbb{C}^{q}\right)$ its operator-valued kernel, defined by

$$
A u(t)=\int_{\mathbb{R}} A(t, s) u(s) d s, u \in C_{0}^{\infty}\left(M ; \mathbb{C}^{p}\right) .
$$

We denote by $\pi_{i}: L^{2}\left(\Sigma ; \mathbb{C}^{2}\right) \rightarrow L^{2}(\Sigma)$ for $i=0,1$ the projection on the first or second component and by $\theta(s)$ the Heaviside function. 
Theorem 6.8. $\operatorname{Let}_{\mathcal{A}}^{ \pm}(t, s)$ be a microlocal decomposition and let

$$
G_{\mathrm{F}}(t, s)=\mathrm{i}^{-1} \pi_{0}\left(\mathcal{U}_{A}^{+}(t, s) \theta(t-s)-\mathcal{U}_{A}^{-}(t, s) \theta(s-t)\right) \pi_{1}^{*} .
$$

Then $G_{\mathrm{F}}: C_{0}^{\infty}(M) \rightarrow C^{\infty}(M)$ is continuous and:

$$
P \circ G_{\mathrm{F}}=G_{\mathrm{F}} \circ P=\mathbb{1} .
$$

Moreover $\mathrm{WF}\left(G_{\mathrm{F}}\right)^{\prime}=\Delta \cup \mathcal{C}_{\mathrm{F}}$, hence $G_{\mathrm{F}}$ is a Feynman inverse.

Proof. The fact that $G_{\mathrm{F}}: C_{0}^{\infty}(M) \rightarrow C^{\infty}(M)$ is continuous follows from Thm. 6.3 . Lemma 5.14 and the fact that $C_{0}^{\infty}(M) \subset C_{\mathrm{b}}^{\infty}\left(\mathbb{R} ; H^{\infty}(\Sigma)\right) \subset C^{\infty}(M)$ continuously. In the rest of the proof we will use freely the time-kernel notation. We will denote by $\rho$ the map $C_{0}^{\infty}(M) \ni u \mapsto\left(u, \mathrm{i}^{-1} \partial_{t} u\right) \in C_{0}^{\infty}\left(M ; \mathbb{C}^{2}\right)$, whose kernel is $\delta(t-s) \rho(s)$.

To prove that $P \circ G_{\mathrm{F}}=\mathbb{1}$, we set $R(t, s)=\mathcal{U}_{A}^{+}(t, s) \theta(t-s)-\mathcal{U}_{A}^{-}(t, s) \theta(s-t)$. Since $\left(\bar{\partial}_{t}-\mathrm{i} A(t)\right) \circ \mathcal{U}_{A}^{ \pm}=0$, we obtain

$$
\begin{aligned}
& \left(\left(\bar{\partial}_{t}-\mathrm{i} A(t)\right) \circ R\right)(t, s)=\mathcal{U}_{A}^{+}(t, s) \delta(t-s)+\mathcal{U}_{A}^{-}(t, s) \delta(s-t) \\
& =\left(c^{+}(s)+c^{-}(s)\right) \delta(t-s)=\mathbb{1}_{\Sigma} \delta(t-s),
\end{aligned}
$$

hence $\left(\bar{\partial}_{t}-\mathrm{i} A(t)\right) \circ R=\mathbb{1}$. This implies that

$$
\pi_{0} \circ\left(\bar{\partial}_{t}-\mathrm{i} A(t)\right) \circ R \circ \pi_{1}^{*}=0, \quad \pi_{0} \circ\left(\bar{\partial}_{t}-\mathrm{i} A(t)\right) \circ R \circ \pi_{1}^{*}=\mathbb{1},
$$

which by an easy computation implies that $P \circ G_{\mathrm{F}}=\mathbb{1}$.

To prove that $G_{\mathrm{F}} \circ P=\mathbb{1}$ we note that

$$
\pi_{1}^{*} \circ P=\mathrm{i}\left(\bar{\partial}_{t}-\mathrm{i} A(t)\right) \circ \rho, \quad \theta( \pm(t-s)) \circ \bar{\partial}_{s}=\bar{\partial}_{s} \circ \theta( \pm(t-s)) \pm \delta(t-s) .
$$

Using then that

$$
\mathcal{U}_{A}^{ \pm} \circ\left(\bar{\partial}_{t}-\mathrm{i} A(t)\right)=0, \quad \mathcal{U}_{A}^{+}(s, s)+\mathcal{U}_{A}^{-}(s, s)=c^{+}(s)+c^{-}(s)=\mathbb{1},
$$

we obtain that $G_{\mathrm{F}} \circ P=\pi_{0} \circ \rho=\mathbb{1}$. Writing $X=(t, x, \tau, \xi) \in T^{*}(\mathbb{R} \times \Sigma) \backslash\{0\}$ we have:

$$
X_{1} \prec X_{2} \Leftrightarrow \tau_{i}= \pm\left(\xi_{i} \cdot h^{-1}\left(t_{i}, x_{i}\right) \xi_{i}\right)^{\frac{1}{2}},\left(x_{1}, \xi_{1}\right)=\phi^{ \pm}\left(t_{1}, t_{2}\right)\left(x_{2}, \xi_{2}\right) .
$$

Using Thm. 6.5 v) this easily implies that $\mathrm{WF}\left(G_{\mathrm{F}}\right)^{\prime}=\Delta \cup \mathcal{C}_{\mathrm{F}}$.

\section{HadAmard States}

In this section we associate to a microlocal decomposition as in Def. 6.4 a unique pure Hadamard state $\omega$. The Cauchy surface two-point functions (see Def. 7.4 are (matrices of) pseudodifferential operators on $\Sigma$. We give the relation between the spacetime two-point functions of $\omega$ and the operators $\mathcal{U}_{A}^{ \pm}(\cdot, \cdot)$ in Def. 6.4 .

We say that a state is regular if its Cauchy surface two-point functions are (matrices) of pseudodifferential operators (in the sense of the calculus on manifolds of bounded geometry). We show that any pure regular Hadamard state is actually associated to a microlocal decomposition.

7.1. Klein-Gordon fields. We start by reviewing classical material about quasifree states for Klein-Gordon fields, see e.g. [DG, KM, HW. We use the complex formalism, based on charged (i.e., complex) fields $\psi, \psi^{*}$, which turns out to be more convenient for our analysis. 
7.1.1. Bosonic quasi-free states. Let $\mathcal{V}$ be a complex vector space, $\mathcal{V}^{*}$ its anti-dual and let us denote $L_{\mathrm{h}}\left(\mathcal{V}, \mathcal{V}^{*}\right)$ the space of hermitian sesquilinear forms on $\mathcal{V}$. A pair $(\mathcal{V}, q)$ consisting of a complex vector space $\mathcal{V}$ and a non-degenerate hermitian form $q$ on $\mathcal{V}$ will be called a phase space. We denote by $U(\mathcal{V}, q)$ the pseudo-unitary group for $(\mathcal{V}, q)$.

As outlined in the introduction, given a phase space $(\mathcal{V}, q)$ one can define the $C C R$ *-algebra $\operatorname{CCR}(\mathcal{V}, q)$ (see e.g. [DG, Sect. 8.3.1]) 6] The (complex) field operators $\mathcal{V} \ni v \mapsto \psi(v), \psi^{*}(v)$, which generate $\operatorname{CCR}(\mathcal{V}, q)$, are anti-linear, resp. linear in $v$ and satisfy the canonical commutation relations

$$
[\psi(v), \psi(w)]=\left[\psi^{*}(v), \psi^{*}(w)\right]=0, \quad\left[\psi(v), \psi^{*}(w)\right]=\bar{v} q w \mathbb{1}, \quad v, w \in \mathcal{V} .
$$

The complex covariances $\Lambda^{ \pm} \in L_{\mathrm{h}}\left(\mathcal{V}, \mathcal{V}^{*}\right)$ of a state $\omega$ on $\operatorname{CCR}(\mathcal{V}, q)$ are defined in terms of the abstract field operators by

$$
\bar{v} \cdot \Lambda^{+} w:=\omega\left(\psi(v) \psi^{*}(w)\right), \quad \bar{v} \cdot \Lambda^{-} w:=\omega\left(\psi^{*}(w) \psi(v)\right), \quad v, w \in \mathcal{V}
$$

Note that $\Lambda^{ \pm} \geq 0$ and $\Lambda^{+}-\Lambda^{-}=q$ by the canonical commutation relations. Conversely if $\Lambda^{ \pm}$are Hermitian forms on $\mathcal{V}$ such that

$$
\Lambda^{+}-\Lambda^{-}=q, \quad \Lambda^{ \pm} \geq 0,
$$

then there is a unique quasi-free state $\omega$ such that 7.1 holds, see e.g. DG, Sect. 17.1].

In order to discuss purity of quasi-free states in terms of their two-point functions, one needs to work in a $C^{*}$-algebraic framework instead.

If $\mathcal{V}_{\mathbb{R}}$ is $\mathcal{V}$ considered as a real vector space and $\sigma=\mathrm{i}^{-1} q$, then $\left(\mathcal{V}_{\mathbb{R}}, \operatorname{Re} \sigma\right)$ is a real symplectic space. We denote by $\mathrm{W}(\mathcal{V}, q)$ the Weyl $C^{*}$-algebra over $\left(\mathcal{V}_{\mathbb{R}}, \operatorname{Re} \sigma\right)$, see e.g. [DG, Sect. 8.5.3], whose generators are denoted by $W(v)$. We still denote by $\omega$ the quasi-free state on $\mathrm{W}(\mathcal{V}, q)$ defined by

$$
\omega(W(v))=\mathrm{e}^{-\frac{1}{2} v \eta v}, \text { for } \eta=\operatorname{Re}\left(\Lambda^{ \pm} \mp \frac{1}{2} q\right),
$$

see [GW1, Sect. 2.3]. By definition $\omega$ is pure if it is pure as a state on the $C^{*}$-algebra $\mathrm{W}(\mathcal{V}, q)$.

Note that 7.2 implies that $\operatorname{Ker}\left(\Lambda^{+}+\Lambda^{-}\right)=\{0\}$, hence $\|v\|_{\omega}^{2}:=\bar{v} \Lambda^{+} v+\bar{v} \Lambda^{-} v$ is a Hilbert norm on $\mathcal{V}$. Denoting by $\mathcal{V}^{\text {cpl }}$ the completion of $\mathcal{V}$ for $\|\cdot\|_{\omega}$, the hermitian forms $q, \Lambda^{ \pm}$extend uniquely to $q^{\mathrm{cpl}}, \Lambda^{ \pm, \mathrm{cpl}}$ on $\mathcal{V}^{\mathrm{cpl}}$, and $\omega$ uniquely extends to a state $\omega^{\mathrm{cpl}}$ on $\operatorname{CCR}\left(\mathcal{V}^{\mathrm{cpl}}, q^{\mathrm{cpl}}\right)$ or $\mathrm{W}\left(\mathcal{V}^{\mathrm{cpl}}, q^{\mathrm{cpl}}\right)$. Note that $q^{\mathrm{cpl}}$ may be degenerate.

If $\mathcal{V}_{1} \subset \mathcal{V}^{\mathrm{cpl}}$ with $\mathcal{V} \subset \mathcal{V}_{1}$ densely for $\|\cdot\|_{\omega}$, then we also obtain unique objects $q_{1}, \Lambda_{1}^{ \pm}, \omega_{1}$ that extend $q, \Lambda^{ \pm}, \omega$.

In the proposition below, we give a characterization of pure quasi-free states. Note that the characterization given in [GW1, Prop. 2.7] was incorrect, unless $\mathcal{V}=\mathcal{V}^{\mathrm{cpl}}$.

Proposition 7.1. The state $\omega$ is pure on $\operatorname{CCR}(\mathcal{V}, q)$ iff there exists $\mathcal{V}_{1} \subset \mathcal{V}^{\text {cpl }}$ with $\mathcal{V} \subset \mathcal{V}_{1}$ densely for $\|\cdot\|_{\omega}$ and projections $c_{1}^{ \pm} \in L\left(\mathcal{V}_{1}\right)$ such that

$$
c_{1}^{+}+c_{1}^{-}=\mathbb{1}, c_{1}^{+*} q_{1} c_{1}^{-}=0, \Lambda_{1}^{ \pm}= \pm q_{1} \circ c_{1}^{ \pm} .
$$

The proof is given in Appendix A.2.

\footnotetext{
${ }^{6}$ See GW1 for the transition between real and complex vector space terminology.
} 
7.1.2. Phase spaces for Klein-Gordon fields. Let $(M, g)$ be a globally hyperbolic spacetime and $P=-\nabla^{a} \nabla_{a}+V(x)$, for $V \in C^{\infty}(M, \mathbb{R})$ a Klein-Gordon operator on $(M, g)$. More generally $P$ can be any formally selfadjoint second order differential operator, whose principal symbol $\sigma_{\mathrm{pr}}(P)$ equals $\xi \cdot g^{-1}(x) \xi$.

We denote by $G_{\text {ret/adv }}$ the retarded/advanced inverses for $P$ and by $G:=G_{\text {ret }}-$ $G_{\text {adv }}$, the Pauli-Jordan commutator. We set

$$
(u \mid v)_{M}:=\int_{M} \bar{u} v d \operatorname{vol}_{g}, u, v \in C_{0}^{\infty}(M) .
$$

The classical phase space associated to $P$ is $(\mathcal{V}, Q)$, where

$$
\mathcal{V}:=\frac{C_{0}^{\infty}(M)}{P C_{0}^{\infty}(M)}, \quad \overline{[u]} \cdot Q[v]:=\mathrm{i}(u \mid G v)_{M} .
$$

Let $\Sigma$ be a Cauchy hypersurface,

$$
\rho_{\Sigma} u:=\left(\begin{array}{c}
\left.u\right|_{\Sigma} \\
\mathrm{i}^{-1} \partial_{n} u \uparrow_{\Sigma}
\end{array}\right)
$$

where $n$ is the future unit normal to $\Sigma$ and $\mathcal{V}_{\Sigma}=C_{0}^{\infty}\left(\Sigma ; \mathbb{C}^{2}\right)$. We equip $\mathcal{V}_{\Sigma}$ with the scalar product

$$
(f \mid g)_{\Sigma}:=\int_{\Sigma} \bar{f}_{0} g_{0}+\bar{f}_{1} g_{1} d \sigma_{\Sigma},
$$

Then $\rho_{\Sigma} G: \mathcal{V} \rightarrow \mathcal{V}_{\Sigma}$ is bijective, it makes thus sense to define $G_{\Sigma}: \mathcal{V}_{\Sigma}=$ $C_{0}^{\infty}\left(\Sigma ; \mathbb{C}^{2}\right) \rightarrow C^{\infty}\left(\Sigma ; \mathbb{C}^{2}\right)$ by the identity:

$$
G=:\left(\rho_{\Sigma} G\right)^{*} G_{\Sigma} \rho_{\Sigma} G,
$$

where the adjoint is taken with respect to the scalar products 7.4 , (7.6). Finally we set

$$
\bar{f} q_{\Sigma} g:=\mathrm{i}\left(f \mid G_{\Sigma} g\right)_{\Sigma}
$$

so that the map:

$$
\rho_{\Sigma} G:(\mathcal{V}, Q) \rightarrow\left(\mathcal{V}_{\Sigma}, q_{\Sigma}\right)
$$

is pseudo-unitary. One can use equivalently either of the above phase spaces. By a computation that uses Stoke's theorem, one has concretely (see e.g. [DG])

$$
q_{\Sigma}=\left(\begin{array}{ll}
0 & 1 \\
1 & 0
\end{array}\right)
$$

By the definition of $G_{\Sigma}$,

$$
\mathbb{1}=G^{*} \rho_{\Sigma}^{*} G_{\Sigma} \rho_{\Sigma} \text { on } G C_{0}^{\infty}(M) .
$$

This also implies $\rho_{\Sigma}=\rho_{\Sigma} G^{*} \rho_{\Sigma}^{*} G_{\Sigma} \rho_{\Sigma}$ on $G C_{0}^{\infty}(M)$. On the other hand, denoting $C_{\mathrm{sc}}^{\infty}(M)$ the space of space-compact smooth functions (i.e. smooth functions whose restriction to $\Sigma$ have compact support), it is well-known that $G C_{0}^{\infty}(M)$ is exactly $\left.\operatorname{Ker} P\right|_{C_{\mathrm{sc}}^{\infty}(M)}$, see e.g. [BGP]. Furthermore, since the Cauchy problem

$$
\left\{\begin{array}{l}
P u=0, \\
\rho_{\Sigma} u=f .
\end{array}\right.
$$

is well-posed in $u \in C_{\mathrm{sc}}^{\infty}(M)$ for any $f \in C_{0}^{\infty}\left(\Sigma ; \mathbb{C}^{2}\right)$, the map $\rho_{\Sigma}:\left.\operatorname{Ker} P\right|_{C_{\mathrm{sc}}^{\infty}(M)} \rightarrow$ $C_{0}^{\infty}\left(\Sigma ; \mathbb{C}^{2}\right)$ is bijective and therefore

$$
\mathbb{1}=\rho_{\Sigma} G^{*} \rho_{\Sigma}^{*} G_{\Sigma} \text { on } C_{0}^{\infty}\left(\Sigma ; \mathbb{C}^{2}\right) .
$$


7.1.3. Cauchy evolution operator. It is useful to introduce the Cauchy evolution operator:

$$
\mathcal{U}_{\Sigma}:=G^{*} \rho_{\Sigma}^{*} G_{\Sigma}
$$

By 7.9 and 7.11, it satisfies $\rho_{\Sigma} \mathcal{U}_{\Sigma}=\mathbb{1}$ on $C_{0}^{\infty}\left(\Sigma ; \mathbb{C}^{2}\right)$ and $\mathcal{U}_{\Sigma} \rho_{\Sigma}=\mathbb{1}$ on Ker $\left.P\right|_{C_{\mathrm{sc}}^{\infty}(M)}$. Moreover, since $G^{*}=-G$ we get $P \mathcal{U}_{\Sigma}=0$ hence for $f \in C_{0}^{\infty}\left(\Sigma ; \mathbb{C}^{2}\right)$, $u=\mathcal{U}_{\Sigma} f$ is the unique solution in $C_{\mathrm{sc}}^{\infty}(M)$ of the Cauchy problem (7.10).

7.1.4. Spacetime two-point functions. We use the phase space defined in (7.5). Let us introduce the assumptions:

$$
\begin{aligned}
\text { i) } & \Lambda^{ \pm}: C_{0}^{\infty}(M) \rightarrow C^{\infty}(M) \\
\text { ii) } & \Lambda^{ \pm} \geq 0 \text { for }(\cdot \mid \cdot)_{M} \text { on } C_{0}^{\infty}(M), \\
\text { iii) } & \Lambda^{+}-\Lambda^{-}=\mathrm{i} G, \\
\text { iv) } & P \Lambda^{ \pm}=\Lambda^{ \pm} P=0 .
\end{aligned}
$$

Note that 7.13 implies that $\Lambda^{ \pm}: \mathcal{E}^{\prime}(M) \rightarrow \mathcal{D}^{\prime}(M)$. Let us set with a slight abuse of notation:

$$
\bar{u} \cdot \Lambda^{ \pm} v:=\left(u \mid \Lambda^{ \pm} v\right), u, v \in C_{0}^{\infty}(M) .
$$

If 7.13 hold, then $\Lambda^{ \pm}$define a pair of complex pseudo-covariances on the phase space $(\mathcal{V}, q)$ defined in $(7.5)$, hence define a unique quasi-free-state on $\operatorname{CCR}(\mathcal{V}, Q)$.

Definition 7.2. A pair of maps $\Lambda^{ \pm}: C_{0}^{\infty}(M) \rightarrow C^{\infty}(M)$ satisfying (7.13) will be called a pair of spacetime two-point functions.

7.1.5. Hadamard condition. By the Schwartz kernel theorem, we can also identify $\Lambda^{ \pm}$with a pair of distributions $\Lambda^{ \pm}\left(x, x^{\prime}\right) \in \mathcal{D}^{\prime}(M \times M)$, and one is especially interested in the subclass of Hadamard states, subject to a condition on the wave front set of $\Lambda^{ \pm}\left(x, x^{\prime}\right)$. We recall that the sets $\mathcal{N}^{ \pm}$were defined in 6.6.1.

Definition 7.3. A pair of two-point functions $\Lambda^{ \pm}$satisfying 7.13 is Hadamard if

$$
\operatorname{WF}\left(\Lambda^{ \pm}\right)^{\prime} \subset \mathcal{N}^{ \pm} \times \mathcal{N}^{ \pm}
$$

This form of the Hadamard condition is taken from [SV, $\mathrm{Ho}$, see also $\mathrm{Wr}$ ] for a review on the equivalent formulations. The original formulation in terms of wave front sets is due to Radzikowski [Ra], who showed its equivalence with older definitions $[\mathrm{KW}$.

7.1.6. Cauchy surface two-point functions. We will need a version of two-point functions acting on Cauchy data of $P$ instead of test functions on $M$.

Let us introduce the assumptions:

$$
\begin{aligned}
\text { i) } & \lambda_{\Sigma}^{ \pm}: C_{0}^{\infty}\left(\Sigma ; \mathbb{C}^{2}\right) \rightarrow C^{\infty}\left(\Sigma ; \mathbb{C}^{2}\right), \\
\text { ii) } & \lambda_{\Sigma}^{ \pm} \geq 0 \text { for }(\cdot \mid \cdot)_{\Sigma}, \\
\text { iii) } & \lambda_{\Sigma}^{+}-\lambda_{\Sigma}^{-}=\mathrm{i} G_{\Sigma} .
\end{aligned}
$$

Definition 7.4. A pair of maps $\lambda_{\Sigma}^{ \pm}$satisfying (7.14) will be called a pair of Cauchy surface two-point functions.

Proposition 7.5. The maps:

$$
\begin{array}{r}
\lambda_{\Sigma}^{ \pm} \mapsto \Lambda^{ \pm}:=\left(\rho_{\Sigma} G\right)^{*} \lambda_{\Sigma}^{ \pm}\left(\rho_{\Sigma} G\right), \\
\Lambda^{ \pm} \mapsto \lambda_{\Sigma}^{ \pm}:=\left(\rho_{\Sigma}^{*} G_{\Sigma}\right)^{*} \Lambda^{ \pm}\left(\rho_{\Sigma}^{*} G_{\Sigma}\right)
\end{array}
$$

are bijective and inverse from one another. Furthermore, $\lambda_{\Sigma}^{ \pm}$are Cauchy surface two-point functions iff $\Lambda^{ \pm}$are two-point functions. 
Prop. 7.5 is proved in GW2] in a slightly more general context.

7.2. Reduction to the model case. In this subsection we consider a KleinGordon operator $P$ on $(M, g)$ in (3.6) satisfying hypotheses $(\mathrm{H}),(\mathrm{M})$ introduced in Subsect. 3.3. We show that the construction of Hadamard states for $P$ can be reduced to the case of a model operator $\tilde{P}$ on $I \times \Sigma$ as introduced in Subsect. 6.2.

We use the notation in Subsect. 3.3. We equip $\tilde{M}=I \times \Sigma$ with the Lorentzian metric $\tilde{g}=-d t^{2}+h_{t}(y) d y^{2}$. We recall that $U=\chi(\tilde{M})$ is an open neighborhood of $\Sigma$ in $M$. We equip $C_{0}^{\infty}(U)$ and $C_{0}^{\infty}(\tilde{M})$ with their canonical scalar products $(\cdot \mid \cdot)_{M}$ and $(\cdot \mid \cdot)_{\tilde{M}} \cdot$

Proposition 7.6. Let us set $W: C_{0}^{\infty}(U) \ni u \mapsto c^{(d-1) / 2} u \circ \chi \in C_{0}^{\infty}(\tilde{M})$. Then the following holds:

(1) There exists $a\left(t, y, \bar{\partial}_{y}\right)$ satisfying the conditions in Subsect. 6.2 for $k_{0}=h_{0}$, such that

$$
\tilde{P}:=\left(W^{-1}\right)^{*} P W^{-1}=\bar{\partial}_{t}^{2}+r(t, y) \bar{\partial}_{t}+a\left(t, y, \bar{\partial}_{y}\right) .
$$

(2) if $\tilde{G}$ is the Pauli-Jordan commutator for $\tilde{P}$ one has $\tilde{G}=W G W^{*}$.

(3) Let $\tilde{\Lambda}^{ \pm}$be the spacetime two-point functions of a Hadamard state $\tilde{\omega}$ for $\tilde{P}$ on $\tilde{M}$. Then there exists a unique Hadamard state $\omega$ for $P$ on $M$ with two-point functions $\Lambda^{ \pm}$such that

$$
\tilde{\Lambda}^{ \pm}=W \Lambda^{ \pm} W^{*}
$$

Moreover $\omega$ is pure iff $\tilde{\omega}$ is pure.

Proof. Without loss of generality we may assume that $\chi=$ Id. Let us first prove (1). We set $\tilde{h}_{t}=c^{2} h_{t}$ so that $g=-c^{2} d t^{2}+\tilde{h}_{t} d x^{2}$. We have:

$$
\begin{aligned}
P= & -|g|^{-\frac{1}{2}} \bar{\partial}_{\mu}|g|^{\frac{1}{2}} g^{\mu \nu} \bar{\partial}_{\nu}+V \\
& =c^{-1}|\tilde{h}|^{-\frac{1}{2}} \bar{\partial}_{t} c^{-1}|\tilde{h}|^{\frac{1}{2}} \bar{\partial}_{t}-c^{-1}|\tilde{h}|^{-\frac{1}{2}} \bar{\partial}_{i} c \tilde{h}^{i j}|\tilde{h}|^{\frac{1}{2}} \bar{\partial}_{j}+V .
\end{aligned}
$$

A routine computation shows that:

$$
\begin{aligned}
& c^{-1}|\tilde{h}|^{-\frac{1}{2}} \bar{\partial}_{t} c^{-1}|\tilde{h}|^{\frac{1}{2}} \bar{\partial}_{t} \\
& =c^{-1}\left(\bar{\partial}_{t}^{2}+c^{-1}|\tilde{h}|^{-\frac{1}{2}} \partial_{t}\left(c|\tilde{h}|^{\frac{1}{2}}\right) \bar{\partial}_{t}+|\tilde{h}|^{-\frac{1}{2}} \partial_{t}\left(|\tilde{h}|^{\frac{1}{2}} \partial_{t} \ln c\right)\right) c^{-1} \\
& =: c^{-1} P_{0}\left(t, y, \bar{\partial}_{t}\right) c^{-1}, \\
& c^{-1}|\tilde{h}|^{-\frac{1}{2}} \bar{\partial}_{i} c \tilde{h}^{i j}|\tilde{h}|^{\frac{1}{2}} \bar{\partial}_{j}+V \\
& =c^{-1}\left(|\tilde{h}|^{-\frac{1}{2}} \bar{\partial}_{i} c \tilde{h}^{i j}|\tilde{h}|^{\frac{1}{2}} \bar{\partial}_{j} c+c^{2} V\right) c^{-1} \\
& =c^{-1}\left(|\tilde{h}|^{-\frac{1}{2}} \bar{\partial}_{i} h^{i j}|\tilde{h}|^{\frac{1}{2}} \bar{\partial}_{j}+|\tilde{h}|^{-\frac{1}{2}} \bar{\partial}_{i}|\tilde{h}|^{\frac{1}{2}} h^{i j} \partial_{j} \ln c+c^{2} V\right) c^{-1} \\
& =: c^{-1} P_{\Sigma}\left(t, y, \bar{\partial}_{y}\right) c^{-1} .
\end{aligned}
$$

Using that $|\tilde{h}|^{\frac{1}{2}}=c^{d}|h|^{\frac{1}{2}}$, we can rewrite these two operators as follows:

$$
\begin{aligned}
P_{0}\left(t, y, \bar{\partial}_{t}\right)= & \bar{\partial}_{t}^{2}+\left(|h|^{-\frac{1}{2}} \partial_{t}|h|^{\frac{1}{2}}+(d+1) \partial_{t} \ln c\right) \bar{\partial}_{t} \\
& +|h|^{-\frac{1}{2}} \partial_{t}|h|^{\frac{1}{2}} \partial_{t} \ln c+d\left(\partial_{t} \ln c\right)^{2}+\partial_{t}^{2} \ln c, \\
P_{\Sigma}\left(t, y, \bar{\partial}_{y}\right)= & |h|^{-\frac{1}{2}} \bar{\partial}_{i} h^{i j}|h|^{\frac{1}{2}} \bar{\partial}_{j}+d \partial_{i} \ln c h^{i j} \bar{\partial}_{j} \\
& +\bar{\partial}_{i} h^{i j} \partial_{j} \ln c-\partial_{i}|h|^{-\frac{1}{2}}|h|^{\frac{1}{2}} h^{i j} \partial_{j} \ln c+d \partial_{i} \ln c h^{i j} \partial_{j} \ln c+c^{2} V .
\end{aligned}
$$


Let now $U$ be the operator of multiplication by $c^{-(d+1) / 2}$. Since

$$
U^{-1} \bar{\partial}_{t} U=\bar{\partial}_{t}-\frac{1}{2}(d+1) \partial_{t} \ln c, U^{-1} \bar{\partial}_{i} U=\bar{\partial}_{i}-\frac{1}{2}(d+1) \partial_{i} \ln c,
$$

we obtain:

$$
c U^{-1} P U c=\bar{\partial}_{t}^{2}+r(t, y) \bar{\partial}_{t}+a\left(t, y, \bar{\partial}_{y}\right) .
$$

By hypotheses $(\mathrm{H}),(\mathrm{M})$ we have $r \in C_{\mathrm{b}}^{\infty}\left(I ; \mathrm{BT}_{0}^{0}\left(\Sigma, h_{0}\right)\right), a \in C_{\mathrm{b}}^{\infty}\left(I ; \operatorname{Diff}^{2}\left(\Sigma, h_{0}\right)\right)$, with principal symbol:

$$
\sigma_{\mathrm{pr}}(a)(t, y, \eta)=\eta \cdot h_{t}^{-1}(y) \eta
$$

If $S: C_{0}^{\infty}(U) \ni u \mapsto c^{(d+3) / 2} u \in C_{0}^{\infty}(\tilde{M})$ we check that $W^{*}=S^{-1}$, hence $c U^{-1} P U c=S P W^{-1}=\tilde{P}$. Since $\tilde{P}$ is selfadjoint for the scalar product $(\cdot \mid \cdot)_{\tilde{M}}$ it follows using $d \operatorname{vol}_{\tilde{g}}=\left|h_{t}\right|^{\frac{1}{2}} d t d y$ that

$$
r=\left|h_{t}\right|^{-\frac{1}{2}} \partial_{t}\left|h_{t}\right|^{\frac{1}{2}}, a\left(t, y, \bar{\partial}_{y}\right)=a^{*}\left(t, y, \bar{\partial}_{y}\right) \text {. }
$$

This completes the proof of (1). From the uniqueness of retarded/advanced inverses we obtain that $\tilde{G}_{\mathrm{ret} / \mathrm{adv}}=W G_{\mathrm{ret} / \mathrm{adv}} W^{*}$, which proves $(2)$.

To prove (3) we use two well-known arguments: the first one is the time-slice property, which means that $\mathcal{V}=\frac{C_{0}^{\infty}(U)}{P C^{\infty}(U)}$, i.e. we can replace $M$ by $U$ in 7.5 , since $U$ is a neighborhood of a Cauchy surface. In other words, a pair of twopoint functions $\Lambda^{ \pm} \in \mathcal{D}^{\prime}(U \times U)$ satisfying $(7.13)$ over $U \times U$ uniquely extends to $\Lambda^{ \pm} \in \mathcal{D}^{\prime}(M \times M)$ satisfying 7.13 over $M \times M$.

The second follows from a result based on Hörmander's propagation of singularities theorem, see [Ra, $[\mathrm{SV}]:$ if $\Lambda^{ \pm}$satisfy (Had) over $U \times U$, then they satisfy (Had) globally, using that $P \Lambda^{ \pm}=\Lambda^{ \pm} P=0$. The proof is complete.

7.3. The pure Hadamard state associated to a microlocal decomposition. In this subsection we consider the model Klein-Gordon operator $\tilde{P}$ obtained from Prop. 7.6. To simplify notation, we denote $\tilde{P}$ by $P, \tilde{M}=I \times \Sigma$ by $M$. We will associate to a microlocal decomposition for $P$ a unique pure Hadamard state. First we need to introduce some more notation.

The level sets $\Sigma_{t}=\{t\} \times \Sigma$ are all Cauchy hypersurfaces. The various objects associated to the Cauchy surface $\Sigma_{t}$, like $\rho_{\Sigma_{t}}, G_{\Sigma_{t}}, \lambda_{\Sigma_{t}}^{ \pm}, \mathcal{U}_{\Sigma_{t}}$ will be simply denoted by $\rho(t), G(t), \lambda^{ \pm}(t), \mathcal{U}(t)$, etc.

Lemma 7.7. One has:

$$
\begin{aligned}
\text { i) } & \lambda_{\omega}^{ \pm}(t)=\mathcal{U}_{A}(s, t)^{*} \lambda_{\omega}^{ \pm}(s) \mathcal{U}_{A}(s, t), \\
i i) & c_{\omega}^{ \pm}(t)=\mathcal{U}_{A}(t, s) c_{\omega}^{ \pm}(s) \mathcal{U}_{A}(s, t), \\
i i i) & q=\mathcal{U}_{A}(s, t)^{*} q \mathcal{U}_{A}(s, t) \\
i v) & \mathcal{U}_{A}(t, s)=\rho(t) G^{*} \rho(s)^{*} G(s) .
\end{aligned}
$$

Proof. It suffices to use the various identities in 7.1.2 7.1.3 and the fact that $\mathcal{U}_{A}(t, s)=\rho(t) \mathcal{U}(s)$.

Theorem 7.8. Let $\left\{\mathcal{U}_{A}^{ \pm}(\cdot, \cdot)\right\}_{(t, s) \in I^{2}}$ be a microlocal decomposition of the evolution $\mathcal{U}_{A}$ as in Subsect. 6.5 and let $\lambda^{ \pm}(t):= \pm q \circ c^{ \pm}(t)$, where $c^{ \pm}(t)$ are defined in Prop. 6.6. Then $\lambda^{ \pm}(t)$ are the Cauchy surface two-point functions of a pure Hadamard state. One has:

$$
\begin{aligned}
\lambda^{ \pm}(t) & =\mathcal{U}_{A}(0, t)^{*} T^{-1}(0)^{*} \pi^{ \pm} T^{-1}(0) \mathcal{U}_{A}(0, t) \\
& =T^{-1}(t)^{*} \mathcal{U}_{C}(0, t)^{*} \pi^{ \pm} \mathcal{U}_{C}(0, t) T^{-1}(t) \\
& =T^{-1}(t)^{*} \pi^{ \pm} T^{-1}(t)+C_{\mathrm{b}}^{\infty}\left(I ; \Psi^{-\infty}(\Sigma)\right) .
\end{aligned}
$$


where $\pi^{ \pm}$are defined in 6.25 and $T(t)$ in 6.15.

Proof. Let us first prove 67.15). From the definition of $c^{ \pm}(t)$ (see 6.26, 6.29), we have:

$$
\begin{aligned}
\lambda^{ \pm}(t) & = \pm q \mathcal{U}_{A}(t, 0) T(0) \pi^{ \pm} T^{-1}(0) \mathcal{U}_{A}(0, t) \\
& = \pm \mathcal{U}_{A}(0, t)^{*} q T(0) \pi^{ \pm} T^{-1}(0) \mathcal{U}_{A}(0, t) \\
& = \pm \mathcal{U}_{A}(0, t)^{*} T^{-1}(0)^{*} \hat{q} \pi^{ \pm} T^{-1}(0) \mathcal{U}_{A}(0, t) \\
& =\mathcal{U}_{A}(0, t)^{*} T^{-1}(0)^{*} \pi^{ \pm} T^{-1}(0) \mathcal{U}_{A}(0, t),
\end{aligned}
$$

where we used successively 6.8, (6.16) and the fact that $\pm \hat{q} \pi^{ \pm}=\pi^{ \pm}$. The second line in 7.15 follows then from 6.14). From 6.17), 6.22 we obtain then

$$
\hat{q}=\mathcal{U}_{C}(0, t)^{*} \hat{q} \mathcal{U}_{C}(0, t)=\mathcal{U}_{\tilde{C}}(0, t)^{*} \hat{q} \mathcal{U}_{\tilde{C}}(0, t)+C_{\mathrm{b}}^{\infty}\left(I, \Psi^{-\infty}(\Sigma)\right) .
$$

Since $\hat{q}$ and $\mathcal{U}_{\tilde{C}}(0, t)$ are diagonal this implies that

$$
\pi^{ \pm}=\mathcal{U}_{\tilde{C}}(0, t)^{*} \pi^{ \pm} \mathcal{U}_{\tilde{C}}(0, t)+C_{\mathrm{b}}^{\infty}\left(I, \Psi^{-\infty}(\Sigma)\right)
$$

which using once more 6.22 gives:

$$
\pi^{ \pm}=\mathcal{U}_{C}(0, t)^{*} \pi^{ \pm} \mathcal{U}_{C}(0, t)+C_{\mathrm{b}}^{\infty}\left(I, \Psi^{-\infty}(\Sigma)\right),
$$

which completes the proof of 7.15 .

To check that $\lambda^{ \pm}(t)$ are the Cauchy surface two-point functions of a pure Hadamard state we work with the Cauchy surface $\Sigma_{0}$. By Prop. 6.6 we know that $c^{+}(0)+c^{-}(0)=\mathbb{1}$ hence condition (7.14) iii) is satisfied. Condition $i$ ) follows from the fact that $c^{ \pm}(0) \in \Psi^{\infty}(\Sigma)$. The positivity condition iii) follows from $(7.15)$. To check the Hadamard condition one can use the arguments in GW2, which we recall for the sake of self-containedness. We have by 6.28:

$$
\mathcal{U}(0) c^{ \pm}(0)=\mathcal{U}_{A}^{ \pm}(\cdot, 0) c^{ \pm}(0) \text { on } \mathcal{E}^{\prime}\left(\Sigma ; \mathbb{C}^{2}\right)
$$

hence

$$
\Lambda^{ \pm}= \pm \mathrm{i} \mathcal{U}(0) c^{ \pm}(0) \rho(0) G= \pm \mathrm{i} \mathcal{U}_{A}^{ \pm}(\cdot, 0) \rho(0) G, \text { on } \mathcal{E}^{\prime}(M)
$$

From Thm. 6.5 this implies that $\mathrm{WF}^{\prime}\left(\Lambda^{ \pm}\right) \subset \mathcal{N}^{ \pm} \times \mathcal{N}$. Since $\Lambda^{ \pm}=\left(\Lambda^{ \pm}\right)^{*}$ we have also $\mathrm{WF}^{\prime}\left(\Lambda^{ \pm}\right) \subset \mathcal{N}^{ \pm} \times \mathcal{N}^{ \pm}$. This shows that the state $\omega$ is Hadamard. The fact that $\omega$ is pure follows from the fact that $c^{ \pm}(0)$ are projections.

We recall from Subsects. 6.4.3. 6.5 that a microlocal decomposition $\mathcal{U}_{A}^{ \pm}(\cdot, \cdot)$ is uniquely obtained from a generator $b(t)$ constructed in Thm. 6.1 as an approximate solution of a Riccati equation. Consequently to any such $b(t)$ corresponds a unique pure Hadamard state.

Definition 7.9. The pure Hadamard state associated to a generator $b(t)$ in Thm. 7.8 will be denoted by $\omega_{b}$.

It is now easy to find the relationship between the spacetime two-point functions $\Lambda^{ \pm}$of $\omega$ and the operators $\mathcal{U}_{A}(t, s)$. As in Subsect. 6.6 we denote by $A(t, s)$ the time kernel of an operator $A$.

Theorem 7.10. Let $\Lambda^{ \pm}$be the spacetime two-point functions of the state $\omega$ constructed in Thm. 7.8. Then:

$$
\mathcal{U}_{A}^{ \pm}(t, s)= \pm\left(\begin{array}{cc}
\mathrm{i} \partial_{s} \Lambda^{ \pm}(t, s) & \Lambda^{ \pm}(t, s) \\
\partial_{t} \partial_{s} \Lambda^{ \pm}(t, s) & \mathrm{i}^{-1} \partial_{t} \Lambda^{ \pm}(t, s)
\end{array}\right)
$$

Consequently we have:

$$
\Lambda^{ \pm}(t, s)= \pm \pi_{0} \mathcal{U}_{A}^{ \pm}(t, s) \pi_{1}^{*},
$$

where $\pi_{i}$ are defined in Subsect. 6.6. 
Proof. Using 6.28 and the identities in Lemma 7.7. Prop. 7.5 we obtain:

$$
\begin{aligned}
\mathcal{U}_{A}^{ \pm}(t, s) & =\mathcal{U}_{A}(t, 0) c^{ \pm}(0) \mathcal{U}_{A}(0, s) \\
& =\rho(t) G^{*} \rho(0)^{*} G(0) c^{ \pm}(0) \rho(0) G^{*} \rho(s)^{*} G(s) \\
& = \pm \mathrm{i} \rho(t) G^{*} \rho(0)^{*} \lambda^{ \pm}(0) \rho(0) G^{*} \rho(s)^{*} G(s) \\
& = \pm \mathrm{i} \rho(t) \Lambda^{ \pm} \rho(s)^{*} G(s)= \pm \rho(t) \Lambda^{ \pm} \rho(s)^{*} q
\end{aligned}
$$

Using $\rho(s)^{*} f=f^{0} \otimes \delta(s)-\mathrm{i} f^{1} \otimes \delta^{\prime}(s)$, this yields:

$$
\mathcal{U}_{A}^{ \pm}(t, s)= \pm\left(\begin{array}{cc}
\mathrm{i} \partial_{s} \Lambda^{ \pm}(t, s) & \Lambda^{ \pm}(t, s) \\
\partial_{t} \partial_{s} \Lambda^{ \pm}(t, s) & \mathrm{i}^{-1} \partial_{t} \Lambda^{ \pm}(t, s)
\end{array}\right),
$$

which completes the proof.

In Subsect. 6.6 we associated to a microlocal decomposition a canonical Feynman inverse $G_{\mathrm{F}}$, see Thm. 6.8. On the other hand, it is well-known (see e.g. [Ra] or [Wr, Thm. 3.4.4] for the complex case) that if $\Lambda^{ \pm}$are the spacetime two-point functions of a Hadamard state $\omega$, then the operator

$$
\mathrm{i}^{-1} \Lambda^{+}+G_{\text {adv }}=\mathrm{i}^{-1} \Lambda^{-}+G_{\text {ret }}
$$

is a Feynman inverse of $P$. It is easy to show that if $\omega$ is the state in Thm. 7.8 then both Feynman inverses are the same.

Proposition 7.11. Let $G_{\mathrm{F}}$ and $\Lambda^{ \pm}$the Feynman inverse and spacetime two-point functions associated to the microlocal decomposition $\left\{\mathcal{U}_{A}^{ \pm}(\cdot, \cdot)\right\}_{(t, s) \in I^{2}}$. Then

$$
G_{\mathrm{F}}=\mathrm{i}^{-1} \Lambda^{+}+G_{\mathrm{adv}}=\mathrm{i}^{-1} \Lambda^{-}+G_{\text {ret }}
$$

Proof. Arguing as in the proof of Thm. 6.8 we see that

$$
G_{\text {ret }}(t, s)=\mathrm{i}^{-1} \pi_{0} \mathcal{U}_{A}(t, s) \pi_{1}^{*} \theta(t-s), G_{\text {adv }}(t, s)=-\mathrm{i}^{-1} \pi_{0} \mathcal{U}_{A}(t, s) \pi_{1}^{*} \theta(s-t) .
$$

Then the proposition follows from the identities 6.31), (7.17).

\subsection{Regular Hadamard states.}

Definition 7.12. A state $\omega$ is regular if $\lambda_{\omega}^{ \pm}(t) \in C^{\infty}\left(\mathbb{R}, \Psi^{\infty}\left(\Sigma ; M_{2}(\mathbb{C})\right)\right)$.

In other words regular states have Cauchy surface two-point functions equal to matrices of pseudodifferential operators. The following lemma shows that it suffices to check the pseudodifferential property for one time $t$.

Lemma 7.13. $\omega$ is regular iff there exists $s \in I$ such that $\lambda_{\omega}^{ \pm}(s) \in \Psi^{\infty}\left(\Sigma ; M_{2}(\mathbb{C})\right)$.

Proof. Assume that $\lambda_{\omega}^{ \pm}(s) \in \Psi^{\infty}\left(\Sigma ; M_{2}(\mathbb{C})\right)$ for some $s \in I$. Then $\lambda_{\omega}^{ \pm}(t)$ is given by Lemma $7.7 i)$. By Thm. 6.3 we can replace $\mathcal{U}_{A}(s, t)$ by $\mathcal{U}_{\tilde{A}}(s, t)$ and then by $\mathcal{U}_{\tilde{C}}(s, t)$, which has a diagonal generator, see 6.19$)$. Then we apply Egorov's Theorem, Thm. 5.15.

Let now $\omega$ be the Hadamard state associated to a microlocal decomposition as in Thm. 7.8, and $\omega_{1}$ another regular state. We denote by $\Lambda^{ \pm}, \Lambda_{1}^{ \pm}, \lambda^{ \pm}(t), \lambda_{1}^{ \pm}(t)$ their respective spacetime and Cauchy surface two-point functions.

Proposition 7.14. A regular state $\omega_{1}$ is Hadamard iff:

$$
\lambda_{1}^{ \pm}(t)-\lambda^{ \pm}(t) \in C^{\infty}\left(\mathbb{R} ; \Psi^{-\infty}\left(\Sigma ; M_{2}(\mathbb{C})\right)\right) .
$$

Proof. It is well known (see e.g. [Ra] or [Wr. Thm. 3.4.4] for the complex case) that $\Lambda^{ \pm}-\Lambda_{1}^{ \pm}$are smoothing operators on $M$, hence $\lambda^{ \pm}(t)-\lambda_{1}^{ \pm}(t)$ are smoothing operators on $\Sigma$. By Lemma 5.13 this yields the $\Rightarrow$ implication. The $\Leftarrow$ implication is immediate. 
7.4.1. Bogoliubov transformations. We work in the setup of 7.1.1. It is well known, see e.g. [DG. Thm. 11.20], that if $\omega, \omega_{1}$ are two pure quasi-free states on $\operatorname{CCR}(\mathcal{V}, q)$, then there exists $u \in U(\mathcal{V}, q)$ such that

$$
\lambda_{1}^{ \pm}=u^{*} \lambda^{ \pm} u
$$

We now examine the form of the operator $u$ if $\omega$ is a pure Hadamard state associated to a microlocal decomposition as in Thm. 7.8 and $\omega_{1}$ is another pure, regular Hadamard state. In the proposition below, we fix the reference time $t=0$. The operator $b(0) \in \Psi^{1}(\Sigma)$ entering in the definition of $\lambda^{ \pm}(0)$ (see formulas 7.15 and 6.26) will be simply denoted by $b$.

Proposition 7.15. Let $\lambda_{1}^{ \pm}(0)$ be the $t=0$ two-point functions of a pure regular Hadamard state $\omega_{1}$. Then there exists $a \in \Psi^{-\infty}(\Sigma)$ such that:

$$
\lambda_{1}^{+}=T^{-1}(0)^{*} U^{*} \pi^{+} U T^{-1}(0), \text { for } U=\left(\begin{array}{cc}
\left(1+a a^{*}\right)^{\frac{1}{2}} & a \\
a^{*} & \left(1+a^{*} a\right)^{\frac{1}{2}}
\end{array}\right) .
$$

Proof. Let us set

$$
\eta_{1}=\lambda_{1}^{+}+\lambda_{1}^{-}, \quad \hat{\eta}_{1}=T^{-1}(0)^{*} \eta_{1} T^{-1}(0) .
$$

Since $\omega_{1}$ is pure, we deduce from Prop. 7.1 and identity 6.16 that $\hat{\eta}_{1}$ satisfies:

$$
\text { i) } \hat{\eta}_{1} \geq 0, \quad \text { ii) } \hat{\eta}_{1} \hat{q} \hat{\eta}_{1}=\hat{q}
$$

where we recall that $\hat{q}=\left(\begin{array}{cc}1 & 0 \\ 0 & -1\end{array}\right)$. We write $\hat{\eta}_{1}$ as $\left(\begin{array}{cc}a & b \\ b^{*} & c\end{array}\right)$, where using 7.18 and the fact that $\hat{\eta}=\mathbb{1}$ we know that $b, \mathbb{1}-a, \mathbb{1}-c \in \Psi^{-\infty}(\Sigma)$. Now 7.20 is equivalent to:

$$
\begin{aligned}
& \left.i^{\prime}\right) \quad a \geq 0, c \geq 0,|(u \mid b v)| \leq(u \mid a u)^{\frac{1}{2}}(v \mid c v)^{\frac{1}{2}}, u, v \in C_{0}^{\infty}(\Sigma), \\
& \left.i i^{\prime}\right) \quad a^{2}=1+b b^{*}, c^{2}=11+b^{*} b, a b-b c=0 .
\end{aligned}
$$

Since $a, c \geq 0$ by $i^{\prime}$ ), the first two equations of $i i^{\prime}$ ) yield

$$
a=\left(\mathbb{1}+b b^{*}\right)^{\frac{1}{2}}, c=\left(1+b^{*} b\right)^{\frac{1}{2}} .
$$

The third equation of $\left.i i^{\prime}\right)$ then holds using the identity

$$
b f\left(b^{*} b\right)=f\left(b b^{*}\right) b, f \text { any Borel function. }
$$

The second condition in $\left.i^{\prime}\right)$ is equivalent to $\left\|\left(1+b b^{*}\right)^{\frac{1}{2}} b\left(\mathbb{1}+b^{*} b\right)^{\frac{1}{2}}\right\| \leq 1$, which holds using again 7.21. This implies that $\hat{\lambda}_{1}:=T^{-1}(0)^{*} \lambda_{1}^{+} T^{-1}(0)$ equals

$$
\hat{\lambda}_{1}=\frac{1}{2}\left(\begin{array}{cc}
\left(1+b b^{*}\right)^{\frac{1}{2}}+\mathbb{1} & b \\
b^{*} & \left(\mathbb{1}+b^{*} b\right)^{\frac{1}{2}}-\mathbb{1}
\end{array}\right) .
$$

Let now

$$
a:=\frac{b}{\sqrt{2}}\left(\left(\mathbb{1}+b^{*} b\right)^{\frac{1}{2}}+\mathbb{1}\right)^{\frac{1}{2}} \in \Psi^{-\infty}(\Sigma) .
$$

Using (7.21) we obtain by an easy computation that

$$
\mathbb{1}+a^{*} a=\frac{1}{2}\left(\left(\mathbb{1}+b^{*} b\right)^{\frac{1}{2}}+\mathbb{1}\right), \mathbb{1}+a a^{*}=\frac{1}{2}\left(\left(\mathbb{1}+b b^{*}\right)^{\frac{1}{2}}+\mathbb{1}\right), b=2 a\left(\mathbb{1}+a^{*} a\right)^{\frac{1}{2}} .
$$

Hence

$$
\hat{\lambda}_{1}=\left(\begin{array}{cc}
1+a a^{*} & a\left(1+a^{*} a\right)^{\frac{1}{2}} \\
\left(\mathbb{1}+a^{*} a\right)^{\frac{1}{2}} a^{*} & a^{*} a
\end{array}\right)=U^{*} \pi^{+} U
$$

for $U$ as in the proposition.

The following theorem shows that any pure regular Hadamard state is actually associated to a microlocal decomposition. 
Theorem 7.16. Let $\omega_{1}$ be a pure regular Hadamard state. Then there exists a generator $b_{1}(t)$ as in Thm. 6.1 such that $\omega_{1}=\omega_{b_{1}}$.

Before proving Thm. 7.16 we need one more lemma.

Lemma 7.17. Let $a \in \Psi^{-\infty}(\Sigma)$ and set $r(a):=\left(11+a a^{*}\right)^{\frac{1}{2}}-a$. Then $r(a)$ is boundedly invertible with $r(a)^{-1} \in \mathbb{1}+\Psi^{-\infty}(\Sigma)$.

Proof. By the polar decomposition theorem, we have $a=u|a|=\left|a^{*}\right| u$, where $u$ is a partial isometry. Moreover, $r(a)=\left(\mathbb{1}+a a^{*}\right)^{\frac{1}{2}}\left(\mathbb{1}-\left(\mathbb{1}+a a^{*}\right)^{-\frac{1}{2}} a\right)$. To prove invertibility it suffices to notice that $\left(\mathbb{1}+a a^{*}\right)^{-\frac{1}{2}} a=\left(\mathbb{1}+\left|a^{*}\right|^{2}\right)^{-\frac{1}{2}}\left|a^{*}\right| u$ has norm $<1$, which is easily checked by using the self-adjoint functional calculus and the fact that $a^{*}$ is bounded. The fact that $r(a)^{-1}-\mathbb{1} \in \Psi^{-\infty}(\Sigma)$ follows by the argument used already to prove Lemma 5.5 .

Proof of Thm. 7.16. From Prop. 7.15, we know that there exists $a \in \Psi^{-\infty}(\Sigma)$ such that 7.19 holds. Let us first try to find some $b_{1} \in \Psi^{1}(\Sigma)$ such that

$$
\lambda_{1}^{+}=\left(T_{1}^{-1}\right)^{*} \pi^{+} T_{1}^{-1},
$$

where $T_{1}$ is defined as in 6.15 with $b=b(0)$ replaced by $b_{1}$. The proof is divided in several steps.

Step 1: we first solve 7.22). Let $r(a)$ be as in Lemma 7.17 and set

$$
z:=r(a)\left(b+b^{*}\right)^{-\frac{1}{2}} \in \Psi^{-\frac{1}{2}}(\Sigma) .
$$

Note that $z^{-1} \in \Psi^{\frac{1}{2}}(\Sigma)$. We claim that

$$
b_{1}:=b+\left(b+b^{*}\right)^{\frac{1}{2}} a^{*} z^{*-1}=b+\Psi^{-\infty}(\Sigma)
$$

solves 7.22. Indeed, if $V_{1}=\left(\begin{array}{cc}\alpha_{1} & \beta_{1} \\ \gamma_{1} & \delta_{1}\end{array}\right)$ the equation

$$
V^{*} \pi^{+} V=V_{1}^{*} \pi^{+} V_{1},
$$

is equivalent to the system

$$
\left\{\begin{array}{r}
\text { i) } \alpha_{1}^{*} \alpha_{1}=\alpha^{*} \alpha \\
\text { ii) } \alpha_{1}^{*} \beta_{1}=\alpha^{*} \beta, \\
\text { iii) } \beta_{1}^{*} \beta_{1}=\beta^{*} \beta .
\end{array}\right.
$$

If $V=U T(b)$ and $V_{1}=T\left(b_{1}\right)$ we have:

$$
\begin{aligned}
\alpha_{1} & =\left(b_{1}+b_{1}^{*}\right)^{-\frac{1}{2}} b_{1}^{*}, \beta_{1}=\left(b_{1}+b_{1}^{*}\right)^{-\frac{1}{2}}, \\
\alpha & =\left(1+a a^{*}\right)^{\frac{1}{2}}\left(b+b^{*}\right)^{-\frac{1}{2}} b^{*}+a\left(b+b^{*}\right)^{-\frac{1}{2}} b, \\
\beta & =\left(1+a a^{*}\right)^{\frac{1}{2}}\left(b+b^{*}\right)^{-\frac{1}{2}}-a\left(b+b^{*}\right)^{-\frac{1}{2}} .
\end{aligned}
$$

Using the operator $z$ introduced in 7.23 we see that

$$
\alpha=z b^{*}+a\left(b+b^{*}\right)^{\frac{1}{2}}, \beta=z .
$$

Note also that:

$$
\begin{aligned}
& r(a) r(a)^{*}+r(a) a^{*}+\operatorname{ar}(a)^{*} \\
& =(r(a)+a)\left(r(a)^{*}+a^{*}\right)-a a^{*}=1 .
\end{aligned}
$$


Hence for $b_{1}$ given by 7.24 we have:

$$
\begin{aligned}
b_{1}+b_{1}^{*} & =\left(b+b^{*}\right)+\left(b+b^{*}\right)^{\frac{1}{2}} a^{*} z^{*-1}+z^{-1} a\left(b+b^{*}\right)^{-1} \\
& =\left(b+b^{*}\right)^{\frac{1}{2}}\left(1+a^{*} r(a)^{*-1}+r(a)^{-1} a\right)\left(b+b^{*}\right)^{\frac{1}{2}} \\
& =z^{-1}\left(r(a) r(a)^{*}+z\left(b+b^{*}\right)^{\frac{1}{2}} a^{*}+a\left(b+b^{*}\right)^{\frac{1}{2}} z^{*}\right) z^{*-1} \\
& =z^{-1}\left(r(a) r(a)^{*}+r(a) a^{*}+\operatorname{ar}(a)^{*}\right) z^{*-1}=z^{-1} z^{*-1}
\end{aligned}
$$

by 7.27 , hence:

$$
\beta_{1}^{*} \beta_{1}=\left(b_{1}+b_{1}^{*}\right)^{-1}=z^{*} z=\beta^{*} \beta,
$$

hence $7.25 \mathrm{iii})$ is satisfied. We also obtain

$$
\begin{aligned}
\alpha_{1}^{*} \beta_{1} & =b_{1}\left(b_{1}+b_{1}^{*}\right)^{-1}=b_{1} z^{*} z \\
& =b z^{*} z+\left(b+b^{*}\right)^{\frac{1}{2}} a^{*} z=\alpha^{*} \beta,
\end{aligned}
$$

hence $7.25 \mathrm{ii})$ is satisfied. Finally we have

$$
\begin{aligned}
\alpha_{1}^{*} \alpha_{1} & =b_{1}\left(b_{1}+b_{1}^{*}\right)^{-1} b_{1}^{*}=b_{1} z^{*} z b_{1}^{*} \\
& =\left(b z^{*}+\left(b+b^{*}\right)^{\frac{1}{2}} a^{*}\right)\left(z b^{*}+a\left(b+b^{*}\right)^{\frac{1}{2}}\right)=\alpha^{*} \alpha,
\end{aligned}
$$

by 7.26 . Therefore $7.25 i)$ is satisfied and $b_{1}$ solves 7.22 .

Step 2: we check that $b_{1}=b_{1}(0)$ satisfies the properties i), ii) and iii) in Thm. 6.1 at $t=0$. First of all $b_{1}=b+\Psi^{-\infty}(\Sigma)$ hence $\left.i\right)$ is satisfied. We claim that

$$
b_{1}+b_{1}^{*} \sim b+b^{*}
$$

(see Subsect. 1.4 for notation), which implies properties $i i$ ), iii) at $t=0$. In fact we have:

$$
\begin{aligned}
b_{1}+b_{1}^{*} & =\left(b+b^{*}\right)^{\frac{1}{2}}\left(1+a^{*} r(a)^{-1 *}+r(a)^{-1} a\right)\left(b+b^{*}\right)^{\frac{1}{2}} \\
& =\left(b+b^{*}\right)^{\frac{1}{2}} r(a)^{-1}\left(r(a) r(a)^{*}+r(a) a^{*}+a r(a)^{*}\right) r(a)^{-1 *}\left(b+b^{*}\right)^{\frac{1}{2}} \\
& =\left(b+b^{*}\right)^{\frac{1}{2}} r(a)^{-1}\left((r(a)+a)\left(r(a)^{*}+a^{*}\right)-a a^{*}\right) r(a)^{-1 *}\left(b+b^{*}\right)^{\frac{1}{2}} \\
& =\left(b+b^{*}\right)^{\frac{1}{2}} r(a)^{-1} r(a)^{-1 *}\left(b+b^{*}\right)^{\frac{1}{2}},
\end{aligned}
$$

which implies 7.28 since $r(a)$ is boundedly invertible by Lemma 7.17

Step 3: we now extend $b_{1}$ into $b_{1}(t)$. We set

$$
b_{1}(t)=b(t)+r_{-\infty}(t)
$$

where $r_{-\infty} \in C^{\infty}\left(\mathbb{R}, \Psi^{-\infty}(\Sigma)\right)$ is chosen such that $r_{-\infty}(0)=b_{1}(0)-b(0)$ and properties $i), i i), i i i)$ are satisfied for all $t \in I$. Then $i v)$ is automatically satisfied also. This completes the proof of the theorem.

\section{Appendix A.}

A.1. Computations for Kerr-de Sitter. We recall now an identity of the kind which is often used in the literature (see e.g. ['N2, Lemma 2.2.1] for the Kerr metric).

Remark A.1. The identity in Lemma A.2 allows to check that the Kerr-de Sitter metrics are smooth Lorentzian metrics on $M$ despite the fact the forms $d \varphi, d \theta$ are singular at the poles of $\mathbb{S}^{2}$. In fact the forms $\sin 2 \theta d \theta$ and $\sin ^{2} \theta d \varphi$ are smooth on $\mathbb{S}^{2}$, since they equal $x d x+y d y$ and $x d y-y d x$ in Cartesian coordinates near the poles, and the standard metric on $\mathbb{S}^{2}$ equals $d \theta^{2}+\sin ^{2} \theta d \varphi^{2}$. 
Lemma A.2. Let

Then:

$$
d \omega^{2}=d \theta^{2}+\frac{1+\alpha \cos ^{2} \theta}{1+\alpha} \sin ^{2} \theta d \varphi^{2}
$$

(1) $d \omega^{2}$ is a smooth Riemannian metric on $\mathbb{S}^{2}$.

(2) One has:

$$
g_{\theta \theta} d \theta^{2}+g_{\varphi \varphi} d \varphi^{2}=\frac{\rho^{2}}{\Delta_{\theta}} d \omega^{2}+\left(\frac{2 M \mathrm{a}^{2} r}{(1+\alpha)^{2} \rho^{2}}+\frac{\mathrm{a}^{2}}{1+\alpha}\right)\left(\sin ^{2} \theta d \varphi\right)^{2} .
$$

Proof. $d \omega^{2}$ is clearly positive definite. We have

$$
d \omega^{2}=\left(d \theta^{2}+\sin ^{2} \theta d \varphi^{2}\right)-\frac{\alpha}{1+\alpha}\left(\sin ^{2} \theta d \varphi\right)^{2} .
$$

The first term is the standard metric on $\mathbb{S}^{2}$, the second term is smooth since $\sin ^{2} \theta d \varphi$ is a smooth 1 -form on $\mathbb{S}^{2}$. Therefore $d \omega^{2}$ is smooth, which proves (1).

A routine computation shows that:

$$
\sigma^{2}=\left(r^{2}+a^{2}\right)(1+\alpha) \rho^{2}+2 M a^{2} r \sin ^{2} \theta
$$

Using this identity in the definition of $g_{\varphi \varphi}$ (see 4.2) we easily obtain (2).

A.1.1. Some classes of functions. The map $I \ni r \mapsto s(r) \in \mathbb{R}$ is bijective. Setting:

$$
\begin{aligned}
&(\mathrm{KdS}) \kappa_{h / c}:=\mp \frac{\partial_{r} \Delta_{r}\left(r_{h / c}\right)}{(1+\alpha)\left(r_{h / c}^{2}+\mathrm{a}^{2}\right)}, \\
&(\mathrm{K}) \quad \kappa_{h}:=\mp \frac{\partial_{r} \Delta_{r}\left(r_{h}\right)}{(1+\alpha)\left(r_{h}^{2}+\mathrm{a}^{2}\right)},
\end{aligned}
$$

which are related to the surface gravities at the Kerr-de Sitter resp. Kerr case, one has:

$(\mathrm{KdS}) \quad\left(r-r_{h / c}\right) \sim \mathrm{e}^{-\kappa_{h / c}|s|}, \partial_{s}^{\alpha}\left(r-r_{h / c}\right) \in O\left(\mathrm{e}^{-\kappa_{h / c}|s|}\right)$, for $s \rightarrow \mp \infty$,

(K) $\left\{\begin{array}{l}\left(r-r_{h}\right) \sim \mathrm{e}^{-\kappa_{h}|s|}, \partial_{s}^{\alpha}\left(r-r_{h}\right) \in O\left(\mathrm{e}^{-\kappa_{h}|s|}\right), \text { for } s \rightarrow-\infty, \\ r \sim s, \partial_{s}^{\alpha} r \in O\left(\langle s\rangle^{1-\alpha}\right), \text { for } s \rightarrow+\infty .\end{array}\right.$

Definition A.3. We set:

$$
\begin{aligned}
T_{\mathrm{KdS}}^{0} & =\left\{f \in C^{\infty}(] r_{h}, r_{c}\left[\times \mathbb{S}^{2}\right): \partial_{r}^{\alpha} \partial_{\omega}^{\beta} f \in O(1)\right\}, \\
T_{\mathrm{K}}^{0,0} & =\left\{f \in C^{\infty}(] r_{h},+\infty[): \partial_{r}^{\alpha} \partial_{\omega}^{\beta} f \in O\left(\langle r\rangle^{-\alpha}\right)\right\} \\
T_{\mathrm{KdS}}^{p} & =\left(r-r_{h}\right)^{p}\left(r-r_{c}\right)^{p} T_{\mathrm{KdS}}^{0}, p \in \mathbb{Z}, \\
T_{\mathrm{K}}^{m, p} & =\langle r\rangle^{m-p}\left(r-r_{h}\right)^{p} T_{\mathrm{K}}^{0,0}, m \in \mathbb{R}, p \in \mathbb{Z} .
\end{aligned}
$$

The following are the images of the above spaces under the change of variable $r \mapsto s(r)$.

Definition A.4. We set:

$$
\begin{aligned}
S_{\mathrm{KdS}}^{p} & =\left\{f \in C^{\infty}\left(\mathbb{R} \times \mathbb{S}^{2}\right): \partial_{s}^{\alpha} \partial_{\omega}^{\beta} f \in O\left(\mathrm{e}^{p \kappa_{h / c}|s|}\right), \pm s<0\right\}, p \in \mathbb{Z}^{*}, \\
S_{\mathrm{KdS}}^{0} & =\left\{f \in C^{\infty}\left(\mathbb{R} \times \mathbb{S}^{2}\right): f \text { bounded }, \partial_{s} f \in S_{\mathrm{KdS}}^{-1}\right\}, \\
S_{\mathrm{K}}^{m, p} & =\left\{f \in C^{\infty}\left(\mathbb{R} \times \mathbb{S}^{2}\right): \partial_{s}^{\alpha} \partial_{\omega}^{\beta} f \in O\left(\mathrm{e}^{p \kappa_{h}|s|}\right), s<0, \partial_{s}^{\alpha} \partial_{\omega}^{\beta} f \in O\left(\langle s\rangle^{m-\alpha}\right), s>0\right\}, p \in \mathbb{Z}^{*}, \\
S_{\mathrm{K}}^{m, 0} & =\left\{f \in C^{\infty}\left(\mathbb{R} \times \mathbb{S}^{2}\right): \partial_{\omega}^{\beta} f \in O\left(\langle s\rangle^{m}\right), \partial_{s} f \in S_{\mathrm{K}}^{m-1,-1}\right\} .
\end{aligned}
$$

Definition A.5. A function $f \in S_{\mathrm{KdS}}^{p}$, resp. $f \in S_{\mathrm{K}}^{m, p}$ is elliptic if $f(s, \omega) \neq 0$ on $\mathbb{R} \times \mathbb{S}^{2}$ and $f^{-1} \in S_{\mathrm{KdS}}^{-p}$, resp. $f^{-1} \in S_{\mathrm{K}}^{-m,-p}$.

The following result is easy to prove (see [Hä, Sect. 9.3]). 
Lemma A.6. (1) $S_{\mathrm{KdS}}^{p_{1}} \times S_{\mathrm{KdS}}^{p_{2}} \subset S_{\mathrm{KdS}}^{p_{1}+p_{2}}$ and $S_{\mathrm{K}}^{m_{1}, p_{1}} \times S_{\mathrm{K}}^{m_{2}, p_{2}} \subset S_{\mathrm{K}}^{m_{1}+m_{2}, p_{1}+p_{2}}$, (2) Set $\tilde{f}(s, \omega)=f(r(s), \omega)$ for $f \in C^{\infty}\left(I \times \mathbb{S}^{2}\right)$. Then if $f \in T_{\mathrm{KdS}}^{p}$, resp. $f \in T_{\mathrm{K}}^{m, p}$ one has $\tilde{f} \in S_{\mathrm{KdS}}^{-p}$, resp. $\tilde{f} \in S_{\mathrm{K}}^{m,-p}$.

From Lemma A.6 we obtain easily the following lemma.

Lemma A.7. One has:
i) $\rho^{2}, r^{2}+\mathrm{a}^{2}$ are elliptic in $S_{\mathrm{KdS}}^{0}$, resp. in $S_{\mathrm{K}}^{2,0}$,
ii) $\sigma^{2}$ is elliptic in $S_{\mathrm{KdS}}^{0}$, resp. in $S_{\mathrm{K}}^{4,0}$,
iii) $\Delta_{\theta}$ is elliptic in $S_{\mathrm{KdS}}^{0}$, resp. in $S_{\mathrm{K}}^{0,0}$,
iv) $\Delta_{r}$ is elliptic in $S_{\mathrm{KdS}}^{-1}$, resp. in $S_{\mathrm{K}}^{2,1}$
v) $F(s):=(1+\alpha)^{2} \frac{\left(r^{2}+\mathrm{a}^{2}\right)^{2}}{\Delta_{r}}$ is elliptic in $S_{\mathrm{KdS}}^{1}$, resp. in $S_{\mathrm{K}}^{2,1}$,
vi) $G(s, \theta):=\frac{\sigma^{2}}{\left(r^{2}+a^{2}\right)^{2} \Delta_{\theta}}$, is elliptic in $S_{\mathrm{KdS}}^{0}$, resp. in $S_{\mathrm{K}}^{0,0}$,
vii) $\tilde{c}^{2}=\frac{\Delta_{r} \Delta_{\theta} \rho^{2}}{(1+\alpha)^{2} \sigma^{2}} \in S_{\mathrm{KdS}}^{-1}$ resp. $\in S_{\mathrm{K}}^{0,-1}$.

Proof. Statements $i), \ldots, i v)$ are routine computations, using Lemma A.6 (2). The remaining statements follow then from Lemma A.6 (1).

In the next lemma we estimate the function $R$ defined in 4.2 .2

Lemma A.8. Let $R=g_{t \varphi} g_{\varphi \varphi}^{-1}$ and set

$$
R_{r}(s, \theta)=\partial_{r} R(r(s), \theta), R_{\theta}(s, \theta):=(\sin 2 \theta)^{-1} \partial_{\theta} R(r(s, \theta)) .
$$

Then:

$$
\begin{aligned}
& R_{r} \in S_{\mathrm{KdS}}^{0}, \text { resp. } \in S_{\mathrm{K}}^{0,-3}, \\
& R_{\theta} \in S_{\mathrm{KdS}}^{-1}, \text { resp. } \in S_{\mathrm{K}}^{-1,-2} .
\end{aligned}
$$

Proof. We have

$$
\begin{aligned}
R(r, \theta) & =-\frac{\mathrm{a}}{\sigma^{2}}\left(\Delta_{r}-\left(r^{2}+\mathrm{a}^{2}\right) \Delta_{\theta}\right) \\
& =\frac{\mathrm{a}}{r^{2}+\mathrm{a}^{2}}\left(1-\frac{\Delta_{r}}{\left(r^{2}+\mathrm{a}^{2}\right) \Delta_{\theta}}\right)\left(1-\frac{\mathrm{a}^{2} \Delta_{r} \sin ^{2} \theta}{\left(r^{2}+\mathrm{a}^{2}\right)^{2} \Delta_{\theta}}\right)^{-1},
\end{aligned}
$$

hence

$$
R=\frac{\mathrm{a}}{r^{2}+\mathrm{a}^{2}}\left(1+R_{1}(r, \theta)\right), R_{1} \in T_{\mathrm{KdS}}^{1}, \text { resp. } R_{1} \in T_{\mathrm{K}}^{1,-1} .
$$

It follows that (using that $R$ depends on $\theta$ only through $\sin ^{2} \theta$ ):

$$
\begin{aligned}
& R_{r}:=\partial_{r} R \in T_{\mathrm{Kds}}^{0}, \text { resp. } \in T_{\mathrm{K}}^{0,-3}, \\
& R_{\theta}:=(\sin 2 \theta)^{-1} \partial_{\theta} R \in T_{\mathrm{KdS}}^{1}, \text { resp. } \in T_{\mathrm{K}}^{1,-2} .
\end{aligned}
$$

Passing to the variable $s$ using Lemma A.6 we obtain A.1.

A.2. Proof of Prop. 7.1. Let us recall that in our notations, $\mathcal{V}^{\mathrm{cpl}}$ is the completion of $\mathcal{V}$ with respect to the Hilbert norm $\|v\|_{\omega}^{2}:=\bar{v} \Lambda^{+} v+\bar{v} \Lambda^{-} v$, and the superscript 'cpl' is also used to denote canonical extensions of various objects on $\mathcal{V}$ to $\mathcal{V}^{\mathrm{cpl}}$.

If $\mathcal{V}=\mathcal{V}^{\mathrm{cpl}}$, then the real symplectic space $(\mathcal{V}, \operatorname{Re} \sigma)$ is complete for the Euclidean norm $(v \eta v)^{\frac{1}{2}}, \eta=\operatorname{Re}\left(\Lambda^{ \pm} \mp \frac{1}{2} q\right)$. In that situation, we know from [DG, Thm. 17.13] that $\omega$ is pure iff $\left(2 \eta^{\mathrm{cpl}}, \operatorname{Re} \sigma^{\mathrm{cpl}}\right)$ is Kähler, i.e. there exists an anti-involution 
$\mathrm{j}_{1} \in S p\left(\mathcal{V}^{\mathrm{cpl}}, \operatorname{Re} \sigma^{\mathrm{cpl}}\right)$ such that $2 \eta^{\mathrm{cpl}}=\operatorname{Re} \sigma^{\mathrm{cpl}} \mathrm{j}_{1}$. This is known to be equivalent to the existence of projections $c^{ \pm}$satisfying

$$
c^{+}+c^{-}=\mathbb{1}, c^{+*} q c^{-}=0, \Lambda^{ \pm}= \pm q \circ c^{ \pm},
$$

as requested, see [GW1, Prop. 2.7].

Let us now treat the general case. We recall from [BR, Thm. 2.3.19] that a state $\omega$ on a $C^{*}$-algebra $\mathfrak{A}$ is pure iff its GNS representation $\left(\mathcal{H}_{\omega}, \pi_{\omega}\right)$ is irreducible, i.e. iff $\mathcal{H}_{\omega}$ does not contain non-trivial closed subspaces invariant under $\pi_{\omega}(\mathfrak{A})$.

For $\mathcal{V}_{1}$ as in the statement of the proposition, we set $\mathfrak{A}_{(1)}=\mathrm{W}\left(\mathcal{V}_{(1)}, q_{(1)}\right)$, and we let $\left(\mathcal{H}_{(1)}, \pi_{(1)}, \Omega_{(1)}\right)$ be the GNS triple for $\left(\mathfrak{A}_{(1)}, \omega_{(1)}\right)$. Using that $\mathcal{V}$ is dense in $\mathcal{V}_{1}$ for $\|\cdot\|_{\omega}$, we first obtain that $\mathcal{H}=\mathcal{H}_{1}, \Omega=\Omega_{1}$ and $\left.\pi_{1}\right|_{\mathfrak{A}}=\pi$.

We also easily obtain that $\pi(\mathfrak{A})$ is strongly dense in $\mathfrak{A}_{1}$. In fact, if $A=$ $\sum_{1}^{N} \lambda_{i} \pi_{1}\left(W\left(v_{i}\right)\right) \in \pi_{1}\left(\mathfrak{A}_{1}\right)$ and $v_{i, n} \in \mathcal{V}$ with $v_{i, n} \rightarrow v_{i}$ for $\|\cdot\|_{\omega}$, we obtain that $A_{n}=\sum_{1}^{N} \lambda_{i} \pi\left(W\left(v_{i, n}\right)\right)$ is bounded by $\sum_{1}^{N}\left|\lambda_{i}\right|$ and converges strongly to $A$ on the dense subspace $\pi(\mathfrak{A}) \Omega$, hence on $\mathcal{H}$.

From this fact we see that a closed subspace $\mathcal{K} \subset \mathcal{H}$ is invariant under $\pi(\mathfrak{A})$ iff it is invariant under $\pi_{1}\left(\mathfrak{A}_{1}\right)$, hence $\omega$ is pure iff its extension $\omega_{1}$ to $\mathfrak{A}_{1}$ is pure.

Therefore, the $\Rightarrow$ direction is shown simply by taking $\mathcal{V}_{1}=\mathcal{V}^{\mathrm{cpl}}$. Conversely, if on a space $\mathcal{V}_{1}$ as in the statement of the proposition, there exist projections $c_{1}^{ \pm}$ satisfying A.2 (with $c_{1}^{ \pm}, \Lambda_{1}^{ \pm}$in place of $c^{ \pm}, \Lambda^{ \pm}$), then an easy computation shows that as identities on $L\left(\mathcal{V}_{1}, \mathcal{V}_{1}^{*}\right)$, one has

$$
c_{1}^{ \pm *} \lambda_{1}^{ \pm} c_{1}^{ \pm}=\lambda_{1}^{ \pm}, \quad c_{1}^{ \pm *} \lambda_{1}^{\mp} c_{1}^{ \pm}=0,
$$

hence $c_{1}^{ \pm}$are bounded for $\|\cdot\|_{\omega}$. Therefore they extend to projections on $\mathcal{V}^{\mathrm{cpl}}$ satisfying A.2. This implies that $\omega^{\mathrm{cpl}}$ is pure, hence $\omega$ is pure.

Acknowledgments. The authors would like to thank Victor Nistor and JeanPhilippe Nicolas for useful conversations. M. W. gratefully acknowledges the FranceStanford Center for Interdisciplinary Studies for financial support and the Department of Mathematics of Stanford University for its kind hospitality. The authors also wish to thank the Erwin Schrödinger Institute in Vienna for its hospitality during the program "Modern theory of wave equations".

\section{REFERENCES}

[ALNV] Ammann, B., Lauter, R., Nistor, V., Vasy, A.: Complex powers and non-compact manifolds, Comm. in PDE 29 (2004), 671-705.

[Av] Avetisyan, Z.: A unified mode decomposition method for physical fields in homogeneous cosmology, Rev. Math. Phys. 26 (2014), 1430001.

[BDH] Brouder, C., Dang, N.V., Hélein, F.: Boundedness and continuity of the fundamental operations on distributions having a specified wave front set, (with a counter example by Semyon Alesker), Stud. Math. 232 (2016), 201-226.

[BR] Bratteli, O., Robinson D. W.: Operator Algebras and Quantum Statistical Mechanics, Volume 1, Springer-Verlag, Berlin, second edition (1987).

[BF1] Brum, M., Fredenhagen, K.: 'Vacuum-like' Hadamard states for quantum fields on curved spacetimes, Class. Quantum Grav. 31 (2014), no. 2, 025024.

[BF2] Brunetti, R., Fredenhagen, K.: Microlocal Analysis and Interacting Quantum Field Theories: Renormalization on Physical Backgrounds, Comm. Math. Phys. 208 (2000), 623-661.

[BGP] Bär, C., Ginoux, N., Pfäffle, F.: Wave equation on Lorentzian manifolds and quantization, ESI Lectures in Mathematics and Physics, EMS, 2007.

[BJ] Brum, M., Jorás, S. E.: Hadamard state in Schwarzschild-de Sitter spacetime, Class. Quantum Grav. 32, no. 1 (2014).

[BS] Bernal, A.M., Sánchez, M.: On smooth Cauchy hypersurfaces and Geroch's splitting theorem, Comm. Math. Phys. 243 (2013), 461-470.

[BT] Brum, M., Them, K.: States of low energy in homogeneous and inhomogeneous expanding spacetimes, Class. Quantum Grav. 30, no. 23 (2013), 235035. 
[Ch] Chazarain, J.: Opérateurs hyperboliques à caractéristiques de multiplicité constante, Annales de l'Institut Fourier 24 (1974), 173-202

[C-B] Choquet-Bruhat, Y.: Hyperbolic partial differential equations on a manifold, In Battelle Rencontres, 1967 Lectures Math. Phys., Benjamin, New York, 1968, 84-106.

[CG] Cheeger, J., Gromov, M.: Bounds on the von Neumann dimension of $L^{2}$-cohomology and the Gauss-Bonnet theorem for open manifolds, J. Diff. Geom. 21 (1985), 1-34.

[CGT] Cheeger, J., Gromov, M., Taylor, M.: Finite propagation speed, kernel estimates for functions of the Laplace operator and the geometry of complete Riemannian manifolds, J. Diff. Geom. 17 (1982), 15-54.

[Da1] Dang, N.V.: Complex powers of analytic functions and meromorphic renormalization in QFT, preprint arXiv: 1503.00995, (2015).

[Da2] Dang, N.V.: Renormalization of quantum field theory on curved spacetimes, a causal approach, Ph.D. thesis, Paris Diderot University, (2013).

[Da3] Dang, N.V.: The extension of distributions on manifolds, a microlocal approach, Ann. Henri Poincaré Online First (2015), 10.1007/s00023-015-0419-8.

[DD] Dappiaggi C., Drago, N.: Constructing Hadamard States via an Extended Møller Operator, Lett. Math. Phys. 106:11 (2016). Read More: http://www.worldscientific.com/doi/abs/10.1142/S0129055X14300015

[DMP1] Dappiaggi, C., Moretti, V., Pinamonti, N.: Distinguished quantum states in a class of cosmological spacetimes and their Hadamard property, J. Math. Phys. 50 (2009), 062304.

[DMP2] Dappiaggi, C., Moretti, V., Pinamonti, N.: Rigorous construction and Hadamard property of the Unruh state in Schwarzschild spacetime, Adv. Theor. Math. Phys. 15 (2011) 355.

[DG] Dereziński, J., Gérard, C.: Mathematics of Quantization and Quantum Fields, Cambridge Monographs in Mathematical Physics, Cambridge University Press, 2013.

[Di] Dimock, J.: Algebras of Local Observables on a Manifold, Comm. Math. Phys. 77 (1980), 219-228.

[DH] Duistermaat, J.J., Hörmander, L.: Fourier integral operators, II. Acta Math. 128 (1972), 183-269.

[El] Eldering, J.: Persistence of non compact normally hyperbolic invariant manifolds in bounded geometry, PhD thesis Utrecht University, 2012.

[FNW] Fulling, S.A., Narcowich, F.J., Wald, R.M.: Singularity structure of the two-point function in quantum field theory in curved spacetime, II, Annals of Physics, 136 (1981), 243-272.

[FV1] Fewster, C.J., Verch, R.: The necessity of the Hadamard condition, Class. Quant. Grav. 30, 235027 (2013).

[FV2] Fewster, C.J., Verch, R.: Algebraic quantum field theory in curved spacetimes, in: Advances in Algebraic Quantum Field Theory, Springer (2015).

[GHV] Gell-Redman, J., Haber, N., Vasy, A.: The Feynman propagator on perturbations of Minkowski space, Comm. Math. Phys. 342, (1) (2016), 333-384.

[GW1] Gérard, C., Wrochna, M.: Construction of Hadamard states by pseudo-differential calculus, Comm. Math. Phys. 325 (2) (2014), 713-755.

[GW2] Gérard, C., Wrochna, M.: Hadamard states for the linearized Yang-Mills equation on curved spacetime, Comm. Math. Phys. 337 (2015), 253-320.

[GW3] Gérard, C., Wrochna, M.: Construction of Hadamard states by characteristic Cauchy problem, Anal. PDE 9, (1) (2016), 111-149.

[Hä] Häfner, D.: Sur la théorie de la diffusion pour l'équation de Klein-Gordon dans la métrique de Kerr, Dissert. Mathematicae 421 (2003), 1-102.

[Ho] Hollands, S.: The Hadamard Condition for Dirac Fields and Adiabatic States on RobertsonWalker spacetimes, Comm. Math. Phys. 216 (2001), 635-661.

[Hö] Hörmander, L.: The analysis of linear partial differential operators I. Distribution Theory and Fourier Analysis, Springer, Berlin Heidelberg New York, 1985.

[HW] Hollands, S., Wald, R.M.: Quantum fields in curved spacetime, in: General Relativity and Gravitation: A Centennial Perspective, Cambridge University Press (2015).

[Ko] Kordyukov, Y.: $L^{p}$-theory of elliptic differential operators on manifolds of bounded geometry, Acta Appl. Math. 23 (1991), 223-260.

[KM] Khavkine, I., Moretti, V.: Algebraic QFT in curved spacetime and quasifree Hadamard states: an introduction, in: Advances in Algebraic Quantum Field Theory, Springer (2015).

[KW] Kay, B.S., Wald, R.M.: Theorems on the uniqueness and thermal properties of stationary, nonsingular, quasifree states on spacetimes with a bifurcate Killing horizon, Phys. Rep. 207, 49 (1991)

[Ju1] Junker, W.: Hadamard States, adiabatic vacua and the construction of physical states for scalar quantum fields on curved spacetime, Rev. Math. Phys. 8, (1996), 1091-1159. 
[Ju2] Junker, W.: Erratum to "Hadamard States, adiabatic vacua and the construction of physical states ...", Rev. Math. Phys. 207 (2002), 511-517.

[JS] Junker, W., Schrohe, E.: Adiabatic Vacuum States on General Space-time Manifolds: Definition, Construction, and Physical Properties, Ann. Henri Poincaré, 3 (2002), 11131181.

[Le] Leray, J.: Hyperbolic Differential Equations, Unpublished Lecture Notes, Prince- ton, 1953.

[Mo] Moretti, V.: Quantum out-states holographically induced by asymptotic flatness: invariance under spacetime symmetries, energy positivity and Hadamard property, Comm. Math. Phys. 279 (2008), 31-75.

[Ol] Olbermann, H.: States of Low Energy on Robertson-Walker Spacetimes, Class. Quant. Grav. 24 (2007), 5011.

[O'N1] O' Neill, B.: Semi-Riemannian Geometry, Academic Press, 1983.

[O'N2] O' Neill, B.: The Geometry of Kerr Black Holes, A K Peters, 1995.

[Ou] Oulghazi, O.: Ph.D. thesis in preparation.

[Ra] Radzikowski, M.: Micro-local approach to the Hadamard condition in quantum field theory on curved spacetime. Comm. Math. Phys. 179 (1996), 529-553.

[RS] Reed M., Simon, B.: Methods of modern mathematical physics II: Fourier analysis, selfadjointness, Academic Press, 1975.

[Ro] Roe, J.: An index theorem on open manifolds I, J. Diff. Geom. 27 (1988), 87-113.

[SV] Sahlmann, H., Verch, R.: Passivity and microlocal spectrum condition, Comm; Math. Phys. 214 (2000), 705-731.

[Sa] Sanders, K.: On the Construction of Hartle-Hawking-Israel States Across a Static Bifurcate Killing Horizon, Lett. Math. Phys. 105, 4 (2015), 575-640.

[Se] Seeley, R.: Complex powers of an elliptic operator, in Singular Integrals, Proc. Symp. Pure Math., AMS, Providence, RI, (1967), 288-307.

[Sh1] Shubin, M.A.: Pseudo-differential operators and spectral theory Springer Verlag 2001.

[Sh2] Shubin, M.A.: Spectral theory of elliptic operators on non-compact manifolds, Astérisque 207 (1992), 37-108.

[ST] Stottmeister, A., Thiemann, T.: The microlocal spectrum condition, initial value formulations and background independence, J. Math. Phys. 57 (2016), 022303.

[Ta] Taylor, M.: Pseudo-differential Operators and Nonlinear PDE, Birkhäuser, 1991.

[Va] Vasy, A.: On the positivity of propagator differences, Ann. Henri Poincaré, doi:10.1007/s00023-016-0527-0 (2016).

[VW] Vasy, A., Wrochna, M.: Quantum fields from global propagators on asymptotically Minkowski and extended de Sitter spacetimes, preprint arXiv:1512.08052, (2015).

[Wa] Wald, R.M.: General Relativity, University of Chicago Press, 1985.

[Wr] Wrochna, M.: Singularities of two-point functions in Quantum Field Theory, Ph.D. thesis, University of Göttingen 2013.

Département de Mathématiques, Université Paris-Sud Xi, 91405 Orsay Cedex, FRANCE

E-mail address: christian.gerard@math.u-psud.fr

Département de Mathématiques, Université Paris-Sud Xi, 91405 Orsay Cedex, France

E-mail address: omar.oulghazi@math.u-psud.fr

Université Grenoble Alpes, Institut Fourier, UMr 5582 CNRS, CS 40700, 38058 Grenoble Cedex 09, France

E-mail address: michal.wrochna@univ-grenoble-alpes.fr 